\title{
The Genus 0 Gromov-Witten Invariants of Projective Complete Intersections
}

\author{
Aleksey Zinger*
}

September 10, 2018

\begin{abstract}
We describe the structure of mirror formulas for genus 0 Gromov-Witten invariants of projective complete intersections with any number of marked points and provide an explicit algorithm for obtaining the relevant structure coefficients. The structural description alone suffices for some qualitative applications, such as vanishing results and the bounds on the growth of these invariants predicted by R. Pandharipande.
\end{abstract}

\section{Contents}

1 Introduction $\quad 2$

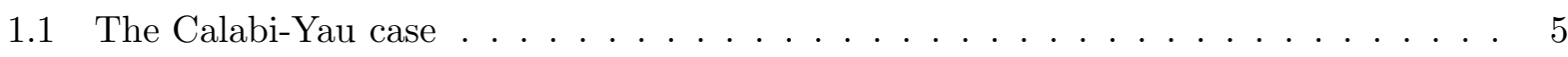

1.2 The projective case . . . . . . . . . . . . . . . 8

2 Main Theorem 9

2.1 An asymptotic expansion . . . . . . . . . . . . . . . . . 10

2.2 One- and two-pointed formulas . . . . . . . . . . . . . . . 12

2.3 Multi-pointed formulas . . . . . . . . . . . . . . . . . 13

2.4 Alternative description of the structure constants . . . . . . . . . . . 18

3 Equivariant GW-invariants

4 Proof of Theorem $\mathbf{A} \quad \mathbf{2 4}$

4.1 Localization Setup . . . . . . . . . . . . . . . . . . . . 24

4.2 Notation and preliminaries . . . . . . . . . . . . . . . 28

4.3 Equivariant one- and two-pointed formulas . . . . . . . . . . . . . . . 29

4.4 Main localization computation . . . . . . . . . . . . . 32

5 Proof of Theorem [1]

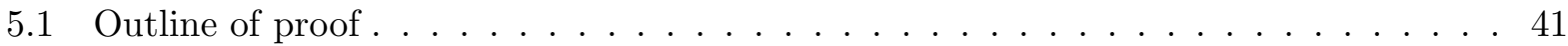

5.2 Bounds on the coefficients of generating functions . . . . . . . . . . . . 43

5.3 Bounds on the structure constants in Theorem $\mathrm{A} \ldots \ldots \ldots \ldots$

A Existence of Asymptotic Expansions

${ }^{*}$ Partially supported by DMS grant 0846978 


\section{Introduction}

Gromov-Witten invariants of a smooth projective variety $X$ are certain counts of curves in $X$. In many cases, these invariants are known or conjectured to possess rather amazing structure which is often completely unexpected from the classical point of view. For example, the genus 0 GWinvariants of a quintic threefold, i.e. a degree 5 hypersurface in $\mathbb{P}^{4}$, are related by a so-called mirror formula to hypergeometric series. This relation was explicitly predicted in [6] and mathematically confirmed in [5], 9], [1], [18], and [19]. In fact, the prediction of [6] has been shown to be a special case of closed formulas for 1-pointed genus $0 \mathrm{GW}$-invariants (counts of curves passing through one constraint) of complete intersections of sufficiently small total multi-degree ([10, 19]). It is shown in 28] that closed formulas for 2-pointed genus $0 \mathrm{GW}$-invariants of hypersurfaces are explicit transforms of the 1-pointed formulas; this is extended to projective complete intersections in [7] and [24].

The classical localization theorem of [3] reduces the computation of genus 0 GW-invariants of projective complete intersections to a sum over decorated graphs. In this paper, we use the method of [27] for breaking such graphs at special nodes to show that closed formulas for $N$-pointed genus 0 GW-invariants of projective complete intersections are explicit transforms of the 1-pointed formulas, with the key link provided by the transform for the 2-pointed invariants obtained in [24]. We show that closed formulas for $N$-pointed genus $0 \mathrm{GW}$-invariants of projective complete intersections, with $N \geq 3$, are linear combinations of $N$-fold products of derivatives of 1-pointed formulas with coefficients that are polynomials of total degree at most $N-3$. While we describe two explicit ways of computing the coefficients of these polynomials, the final formulas become rather complicated as $N$ increases. Nevertheless, our qualitative description of generating functions for $N$-pointed GW-invariants as linear combinations of $N$-fold products of derivatives leads to some simple-to-state qualitative results concerning these invariants; see Theorems 1 and 2 below.

Throughout the paper $N \geq 3, n \geq 2$, and $l \geq 0$ will be fixed integers and

$$
\mathbf{a} \equiv\left(a_{k}\right)_{k=1,2, \ldots, l} \equiv\left(a_{1}, \ldots, a_{l}\right)
$$

a tuple of positive integers, with $N$ and a denoting the number of marked points and the multidegree of a fixed complete intersection $X_{\mathbf{a}} \subset \mathbb{P}^{n-1}$, respectively. Let

$$
\begin{gathered}
{[N]=\{1,2, \ldots, N\},} \\
|\mathbf{a}|=\sum_{k=1}^{k=l} a_{k}, \quad\|\mathbf{a}\|=\sum_{k=1}^{k=l} k a_{k}, \quad\langle\mathbf{a}\rangle=\prod_{l=1}^{l=k} a_{k}, \quad \mathbf{a}^{\mathbf{a}}=\prod_{k=1}^{k=l} a_{k}^{a_{k}}, \quad \mathbf{a} !=\prod_{k=1}^{k=l} a_{k} !, \quad \nu_{\mathbf{a}}=n-|\mathbf{a}| .
\end{gathered}
$$

For any nonnegative integer $d$, we denote by $\overline{\mathfrak{M}}_{0, N}\left(X_{\mathbf{a}}, d\right)$ the moduli spaces of genus 0 degree $d$ $N$-marked stable maps to $X_{\mathbf{a}}$. For each $s=1, \ldots, N$, let

$$
\mathrm{ev}_{s}: \overline{\mathfrak{M}}_{0, N}\left(X_{\mathbf{a}}, d\right) \longrightarrow X_{\mathbf{a}}, \quad \psi_{s} \equiv c_{1}\left(L_{s}^{*}\right) \in H^{2}\left(\overline{\mathfrak{M}}_{0, N}\left(X_{\mathbf{a}}, d\right)\right),
$$

be the evaluation map and the first chern of the universal tangent line bundle at the $s$-th marked point. Denote by $H \in H^{2}\left(\mathbb{P}^{n-1}\right)$ denote the hyperplane class. 
The main theorem of this paper, Theorem $\mathrm{A}$ in Section 2.3. provides closed formulas for generating functions for genus $0 \mathrm{GW}$-invariants

$$
\left\langle\tau_{b_{1}} H^{c_{1}}, \ldots, \tau_{b_{N}} H^{c_{N}}\right\rangle_{0, d}^{X_{\mathbf{a}}} \equiv \int_{\left[\overline{\mathfrak{M}}_{0, N}\left(X_{\mathbf{a}}, d\right)\right]^{v i r}} \prod_{s=1}^{s=N}\left(\psi_{s}^{b_{s}} \operatorname{ev}_{s}^{*} H^{c_{s}}\right)
$$

of a complete intersection $X_{\mathbf{a}} \subset \mathbb{P}^{n-1}$ of multi-degree $\mathbf{a}$ with $|\mathbf{a}| \leq n$. The precise statement of these formulas is quite involved and is thus deferred until Section 2 . We instead begin by describing some qualitative corollaries of Theorem $\mathrm{A}$. Theorems 1 and 2, and special cases, Theorems 3 and 4 .

Theorem 1. If $n \in \mathbb{Z}^{+}, \mathbf{a} \in\left(\mathbb{Z}^{+}\right)^{l}$, and $X_{\mathbf{a}} \subset \mathbb{P}^{n-1}$ is a complete intersection of multi-degree $\mathbf{a}$, there exists $C_{\mathbf{a}} \in \mathbb{R}^{+}$such that

$$
\left|\left\langle b_{1} ! \tau_{b_{1}} H^{c_{1}}, \ldots, b_{N} ! \tau_{b_{N}} H^{c_{N}}\right\rangle_{0, d}^{X_{\mathbf{a}}}\right| \leq N ! C_{\mathbf{a}}^{N+d} \quad \forall N \in \mathbb{Z}^{+}, d, b_{1}, \ldots, b_{N}, c_{1}, \ldots, c_{N} \in \mathbb{Z} .
$$

This bound holds for $d=0$, since

$$
\begin{aligned}
\left\langle\tau_{b_{1}} H^{c_{1}}, \ldots, \tau_{b_{N}} H^{c_{N}}\right\rangle_{0,0}^{X_{\mathbf{a}}} & =\langle\mathbf{a}\rangle\left(\int_{\mathbb{P}^{n-1}} H^{c_{1}+\ldots+c_{N}+l}\right)\left(\int_{\overline{\mathcal{M}}_{0, N}} \psi_{1}^{b_{1}} \ldots \psi_{N}^{b_{N}}\right) \\
& =\langle\mathbf{a}\rangle \delta_{c_{1}+\ldots+c_{N}, n-1-l}\left(\begin{array}{c}
N-3 \\
b_{1}, \ldots, b_{N}
\end{array}\right) \quad \forall c_{1}, \ldots, c_{N} \geq 0,
\end{aligned}
$$

where $\overline{\mathcal{M}}_{0, N}$ is the Deligne-Mumford moduli space of genus 0 curves with $N$ marked points. For $d \in \mathbb{Z}^{+}$, this theorem is proved in Section 5, with the hardest cases $(|\mathbf{a}| \leq n, N \geq 3)$ deduced from Theorem A, It in particular implies that for every Calabi-Yau complete intersection threefold $X_{\mathbf{a}} \subset \mathbb{P}^{n-1}(|\mathbf{a}|=n, l=n-4)$ there exists $C \in \mathbb{R}^{+}$such that

$$
\left|\langle\rangle_{0, d}^{X_{\mathbf{a}}}\right| \leq \frac{N ! C^{N}}{d^{N}} \cdot C^{d} \quad \forall d, N \in \mathbb{Z}^{+}
$$

for $N \leq 2$, this bound also follows from the one-point mirror formulas. According to [21, Theorem 1 ] and [12, Theorem 1] should imply such bounds in all genera via [20]; in turn, the latter imply that generating functions for GW-invariants of any genus have positive radii of convergence, as expected from physical considerations.

Theorem 2. Suppose $n, N \in \mathbb{Z}^{+}$with $N \geq 3$, a $\in\left(\mathbb{Z}^{+}\right)^{l}, X_{\mathbf{a}} \subset \mathbb{P}^{n-1}$ is a complete intersection of multi-degree $\mathbf{a}$, and $\left(b_{s}\right)_{s \in[N]}$ and $\left(c_{s}\right)_{s \in[N]}$ are $N$-tuples of nonnegative integers. If there exists $S \subset[N]$ such that $b_{s}+c_{s}<\nu_{\mathbf{a}}$ for every $s \in S$ and $\sum_{s \in S} b_{s}>N-3$, then

$$
\left\langle\tau_{b_{1}} H^{c_{1}}, \ldots, \tau_{b_{N}} H^{c_{N}}\right\rangle_{0, d}^{X_{\mathbf{a}}}=0 .
$$

This theorem is an immediate consequence of Theorem $\mathrm{A}$; see Remark 5.1. Because of the condition on $b_{s}$, the assumptions of this theorem are never satisfied if $\left|\nu_{\mathbf{a}}\right|=0,1$ (Calabi-Yau and borderline Fano cases). For the same reason, it is most useful if $|\mathbf{a}|=0$ (projective case). For example,

$$
\langle\underbrace{\tau_{b} H^{n-b}, \ldots, \tau_{b} H^{n-b}}_{N-2}, \cdot, \cdot\rangle_{0, d}^{\mathbb{P}^{n}}=0 \quad \forall N \geq 3, b=1,2, \ldots, n .
$$


The $\mathbb{P}^{1}$-case of (1.2) follows from the dilaton relation [15, p527]. For $n \geq 2, \tau_{b} H^{n-b}$ is not a divisor on $\overline{\mathfrak{M}}_{0, N}\left(\mathbb{P}^{n}, d\right)$ and there appears to be no direct geometric reason for the vanishing (1.2).

Theorems 1 and 2 suggest the following conjectures. Theorem 1 establishes the first conjecture for projective complete intersections and $g=0$. The approach of [21] should remove the genus restriction and establish the dependence of $C_{X, g}$ on $g$ and even on $X$. Theorem 2 establishes the second conjecture for projective complete intersections.

Conjecture 1. If $(X, \omega)$ is a compact symplectic manifold, $g \in \mathbb{Z}$, and $H_{1}, \ldots, H_{k} \in H^{*}(X)$, then there exists $C_{X, g} \in \mathbb{R}^{+}$such that

$$
\left|\left\langle b_{1} ! \tau_{b_{1}} H_{c_{1}}, \ldots, b_{N} ! \tau_{b_{N}} H_{c_{N}}\right\rangle_{g, \beta}^{X}\right| \leq N ! C_{X, g}^{N+\langle\omega, \beta\rangle} \quad \forall \beta \in H_{2}(X), N, b_{s} \geq 0, c_{s} \in[k] .
$$

Conjecture 2. Let $(X, \omega)$ be a compact monotone symplectic manifold with minimal chern number $\nu 1$ If $N \geq 3,\left(b_{s}\right)_{s \in[N]}$ and $\left(c_{s}\right)_{s \in[N]}$ are $N$-tuples of nonnegative integers, and $H_{s} \in H^{2 c_{s}}(X)$ for every $s \in[N]$, then

$$
\left\langle\tau_{b_{1}} H_{1}, \ldots, \tau_{b_{N}} H_{N}\right\rangle_{0, \beta}^{X}=0
$$

if there exists $S \subset[N]$ such that $b_{s}+c_{s}<\nu_{\mathbf{a}}$ for every $s \in S$ and $\sum_{s \in S} b_{s}>N-3$.

The genus $0 \mathrm{GW}$-invariants of a complete intersection $X_{\mathbf{a}} \subset \mathbb{P}^{n-1}$ are related to certain twisted GW-invariants of $\mathbb{P}^{n-1}$. Let

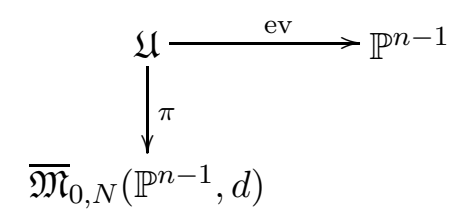

be the universal curve over $\overline{\mathfrak{M}}_{0, N}\left(\mathbb{P}^{n-1}, d\right)$. The GW-invariants of (1.1) then satisfy

$$
\left\langle\tau_{b_{1}} H^{c_{1}}, \ldots, \tau_{b_{N}} H^{c_{N}}\right\rangle_{0, d}^{X_{\mathbf{a}}}=\int_{\bar{M}_{0, N}\left(\mathbb{P}^{n-1}, d\right)} \prod_{k=1}^{k=l} e\left(\pi_{*} \operatorname{ev}^{*} \mathcal{O}_{\mathbb{P}^{n-1}}\left(a_{k}\right)\right) \prod_{s=1}^{s=N}\left(\psi_{s}^{b_{s}} \operatorname{ev}_{s}^{*} H^{c_{s}}\right)
$$

Since the moduli space $\overline{\mathfrak{M}}_{0, N}\left(\mathbb{P}^{n-1}, d\right)$ is a smooth stack (orbifold) and

$$
\bigoplus_{k=1}^{k=l} \pi_{*} \operatorname{ev}^{*} \mathcal{O}_{\mathbb{P}^{n-1}}\left(a_{k}\right) \longrightarrow \overline{\mathfrak{M}}_{0, N}\left(\mathbb{P}^{n-1}, d\right)
$$

is a locally free sheaf, i.e. the sheaf of sections of a vector orbi-bundle $\mathcal{V}_{d}$ over $\overline{\mathfrak{M}}_{0, N}\left(\mathbb{P}^{n-1}, d\right)$, the right-hand side of (1.3) is well-defined; its computation will be the main focus of this paper. In (2.1), we combine all GW-invariants (1.3) with fixed $N$ into a generating function. We show that for $N \geq 3$ this generating function is a certain transform of the $N=1$ generating function.

The general principles of this paper are valid for all a, but the explicit expressions for the transforms apply only for $\nu_{\mathbf{a}} \geq 0$. As the known 1-pointed (and 2-pointed) formulas, they involve the

\footnotetext{
${ }^{1}$ Thus, $c_{1}(X)=\lambda[\omega] \in H^{2}(X ; \mathbb{R})$ for some $\lambda \in \mathbb{R}^{+}$and $\nu$ is the minimal value of $c_{1}(X)$ on the homology classes representable by non-constant $J$-holomorphic maps $S^{2} \longrightarrow X$ for every $\omega$-compatible almost complex structure on $X$.
} 
hypergeometric series

$$
F(w, q) \equiv \sum_{d=0}^{\infty} q^{d} \frac{w^{\nu_{\mathbf{a}} d} \prod_{k=1}^{k=l} \prod_{r=1}^{a_{k} d}\left(a_{k} w+r\right)}{\prod_{r=1}^{r=d}\left((w+r)^{n}-w^{n}\right)} \cdot 2
$$

This power series in $q$ is an element of $1+q \mathbb{Q}(w)[[q]]$ such that the coefficient of each power of $q$ is holomorphic at $w=0$. The subgroup

$$
\mathcal{P} \subset 1+q \mathbb{Q}(w)[[q]]
$$

of such power series is preserved by the operator

$$
\mathbf{M}: 1+q \mathbb{Q}(w)[[q]] \longrightarrow 1+q \mathbb{Q}(w)[[q]], \quad\{\mathbf{M} H\}(w, q)=\left\{1+\frac{q}{w} \frac{\mathrm{d}}{\mathrm{d} q}\right\}\left(\frac{H(w, q)}{H(0, q)}\right)
$$

We define $I_{c} \in 1+q \mathbb{Q}[[q]]$ for $c=0,1, \ldots$ and $J \in q \mathbb{Q}[[q]]$ by

$$
\begin{aligned}
& I_{c}(q)= \begin{cases}1, & \text { if }|\mathbf{a}|<n ; \\
\left\{\mathbf{M}^{c} F\right\}(0, q), & \text { if }|\mathbf{a}|=n ;\end{cases} \\
& J(q)= \begin{cases}0, & \text { if }|\mathbf{a}| \leq n-2 ; \\
\mathbf{a} ! q, & \text { if }|\mathbf{a}|=n-1 ; \\
\frac{1}{I_{0}(q)} \sum_{d=1}^{\infty} q^{d}\left(\frac{\prod_{k=1}^{k=l}\left(a_{k} d\right) !}{(d !)^{n}}\left(\sum_{k=1}^{k=l} \sum_{r=d+1}^{a_{k} d} \frac{a_{k}}{r}\right)\right), & \text { if }|\mathbf{a}|=n .\end{cases}
\end{aligned}
$$

The power series $J(q)$ is the coefficient of $w$ in the power series expansion of $F(w, q) / I_{0}(q)$ at $w=0$; thus, $I_{1}(q)=1+q \frac{\mathrm{d}}{\mathrm{d} q} J(q)$ if $|\mathbf{a}| \neq n-1$. Similarly to the 1- and 2-pointed cases, the explicit expressions of Theorem $\mathrm{A}$ for generating functions for $N \geq 3$ involve the power series $I_{0}, I_{1}, \ldots, I_{n-l}$ and $J$; see Section 1.1 for some examples.

The author would like to thank D. Maulik, R. Pandharipande, and V. Shende for many enlightening discussions related to this paper.

\subsection{The Calabi-Yau case}

If $|\mathbf{a}|=n, X_{\mathbf{a}}$ is a Calabi-Yau $(n-1-l)$-fold and the virtual dimension of $\overline{\mathfrak{M}}_{0, N}\left(X_{\mathbf{a}}, d\right)$ and of the space of $N$-marked rational curves in $X_{\mathbf{a}}$,

$$
\operatorname{dim}^{\text {vir }} \overline{\mathfrak{M}}_{0, N}\left(X_{\mathbf{a}}, d\right)=n-4-l+N,
$$

is independent of $d$. If $c_{1}, \ldots, c_{N}$ are nonnegative integers such that

$$
c_{1}+\ldots+c_{N}=n-4-l+N,
$$

the corresponding genus 0 degree $d \mathrm{GW}$-invariant of $X_{\mathbf{a}}$,

$$
N_{d}^{X_{\mathbf{a}}}\left(c_{1}, \ldots, c_{N}\right) \equiv \int_{\left[\overline{\mathfrak{M}}_{0, N}\left(X_{\mathbf{a}}, d\right)\right] v i r}\left(\operatorname{ev}_{1}^{*} H^{c_{1}}\right) \ldots\left(\operatorname{ev}_{N}^{*} H^{c_{N}}\right),
$$

\footnotetext{
${ }^{2}$ For the purposes of Theorems 3 and 4 the term $w^{n}$ can be dropped from the definition of $F$.
} 
is a rational number. These numbers define BPS states of $X_{\mathbf{a}}$ via [17, (2)], that are intended to be virtual counts of curves (rather than maps) and are conjectured to be integer (see also Footnote 5). For a sufficiently small value of the degree $d$, the corresponding BPS number is known to be the number of rational degree $d$ curves in a general complete intersection of multi-degree a that pass through general linear subspaces of codimensions $c_{1}, \ldots, c_{N}$.

Theorem A yields fairly simple closed formulas for the numbers (1.6) with $N=3,4$. Theorem 3 below follows immediately from (1.3), (2.1), (2.35), (2.20), (2.18), (2.40), (2.42), (2.29), (2.36), (2.41), (2.3), (2.23), and (2.25) 3

Theorem 3. Suppose $n \in \mathbb{Z}^{+}, X_{\mathbf{a}} \subset \mathbb{P}^{n-1}$ is a nonsingular Calabi-Yau complete intersection of multi-degree $\mathbf{a}, I_{c}$ and $J$ are given by (1.5), and $Q=q \cdot e^{J(q)} \in q \mathbb{Q}[[q]]$. If $c_{1}, c_{2}, c_{3}$ are nonnegative integers such that $c_{1}+c_{2}+c_{3}=n-1-l$, then

$$
\sum_{d=0}^{\infty} Q^{d} N_{d}^{X_{\mathbf{a}}}\left(c_{1}, c_{2}, c_{3}\right)=\frac{\langle\mathbf{a}\rangle}{\left(1-\mathbf{a}^{\mathbf{a}} q\right) I_{0}(q)^{2} \prod_{s=1}^{s=3} \prod_{c=1}^{c=c_{s}} I_{c}(q)}
$$

If $c_{1}, c_{2}, c_{3}, c_{4}$ are nonnegative integers such that $c_{1}+c_{2}+c_{3}+c_{4}=n-l$, then

$$
\begin{array}{r}
\sum_{d=0}^{\infty} Q^{d} N_{d}^{X_{\mathbf{a}}}\left(c_{1}, c_{2}, c_{3}, c_{4}\right)=\frac{\langle\mathbf{a}\rangle}{\left(1-\mathbf{a}^{\mathbf{a}} q\right) I_{0}^{2}(q) \prod_{s=1}^{s=4} \prod_{c=1}^{c=c_{s}} I_{c}(q)}\left\{\frac{n-l-2 c_{4}}{2}\left(\frac{\mathbf{a}^{\mathbf{a}} q}{1-\mathbf{a}^{\mathbf{a}} q}-2 \frac{I_{0}^{\prime}(q)}{I_{0}(q)}\right)\right. \\
\left.+\sum_{s=1}^{s=4} \frac{\mathcal{S}_{c_{s}}^{\prime}(q)}{\mathcal{S}_{c_{s}}(q)}-\frac{\mathcal{S}_{c_{1}+c_{2}}^{\prime}(q)}{\mathcal{S}_{c_{1}+c_{2}}(q)}-\frac{\mathcal{S}_{c_{1}+c_{3}}^{\prime}(q)}{\mathcal{S}_{c_{1}+c_{3}}(q)}-\frac{\mathcal{S}_{c_{2}+c_{3}}^{\prime}(q)}{\mathcal{S}_{c_{2}+c_{3}}(q)}\right\}
\end{array}
$$

where' denotes the operator $q \frac{\mathrm{d}}{\mathrm{d} q}$ and $\mathcal{S}_{c}=I_{1}^{c-1} I_{2}^{c-2} \ldots I_{c}^{0}$.

Since $J(q) \in q \mathbb{Q}[[q]]$, there exists $\tilde{J}(Q) \in Q \mathbb{Q}[[Q]]$ such that $q=Q e^{\tilde{J}(Q)}$. Thus, the relations (1.7) and (1.8) determine the numbers $N_{d}^{X_{\mathbf{a}}}\left(c_{1}, c_{2}, c_{3}\right)$ and $N_{d}^{X_{\mathbf{a}}}\left(c_{1}, c_{2}, c_{3}, c_{4}\right)$, respectively. Since

$$
\frac{\mathcal{S}_{c}^{\prime}(q)}{\mathcal{S}_{c}(q)}=\frac{\mathcal{S}_{n-l-c}(q)}{\mathcal{S}_{n-l-c}(q)}-\frac{n-l-2 c}{2}\left(\frac{\mathbf{a}^{\mathbf{a}} q}{1-\mathbf{a}^{\mathbf{a}} q}-2 \frac{I_{0}^{\prime}(q)}{I_{0}(q)}\right) \quad \forall c=0,1, \ldots, n-l
$$

by (2.23)-(2.25) and (2.3), (1.8) is equivalent to

$$
\begin{aligned}
\sum_{d=0}^{\infty} Q^{d} N_{d}^{X_{\mathbf{a}}}\left(c_{1}, c_{2}, c_{3}, c_{4}\right)= & \frac{\langle\mathbf{a}\rangle}{\left(1-\mathbf{a}^{\mathbf{a}} q\right) I_{0}^{2}(q) \prod_{s=1}^{s=4} \prod_{c=1}^{c=c_{s}} I_{c}(q)}\left\{c_{1}\left(\frac{\mathbf{a}^{\mathbf{a}} q}{1-\mathbf{a}^{\mathbf{a}} q}-2 \frac{I_{0}^{\prime}(q)}{I_{0}(q)}\right)\right. \\
& \left.+\sum_{s=1}^{s=4} \frac{\mathcal{S}_{c_{s}}^{\prime}(q)}{\mathcal{S}_{c_{s}}(q)}-\frac{\mathcal{S}_{c_{1}+c_{2}}^{\prime}(q)}{\mathcal{S}_{c_{1}+c_{2}}(q)}-\frac{\mathcal{S}_{c_{1}+c_{3}}^{\prime}(q)}{\mathcal{S}_{c_{1}+c_{3}}(q)}-\frac{\mathcal{S}_{c_{1}+c_{4}}^{\prime}(q)}{\mathcal{S}_{c_{1}+c_{4}}(q)}\right\} .
\end{aligned}
$$

By (1.9), RHS of (1.8) is symmetric in $c_{1}, c_{2}, c_{3}, c_{4}$, as expected. By (1.10),

$$
N_{d}^{X_{\mathrm{a}}}\left(c_{1}, c_{2}, c_{3}, c_{4}\right)=0 \quad \text { if } \quad 0 \in\left\{c_{1}, c_{2}, c_{3}, c_{4}\right\},
$$

\footnotetext{
${ }^{3}(2.40)$ is needed for (1.7) only; (2.42), (2.29), (2.36), (2.41), and (2.25) are needed for (1.8) only
} 
as expected. By (1.7), (2.23), (2.24), and (2.3),

$$
N_{d}^{X_{\mathbf{a}}}\left(c_{1}, c_{2}, c_{3}\right)=\left\{\begin{array}{ll}
\langle\mathbf{a}\rangle, & \text { if } d=0 ; \\
0, & \text { if } d>0 ;
\end{array} \quad \text { if } \quad 0 \in\left\{c_{1}, c_{2}, c_{3}\right\} .\right.
$$

Since $I_{1}(q)=1+q \frac{\mathrm{d}}{\mathrm{d} q} J(q)$, (1.7) and (1.10) immediately give

$$
d N_{d}\left(c_{2}, c_{3}, c_{4}\right)=N_{d}^{X_{\mathbf{a}}}\left(1, c_{2}, c_{3}, c_{4}\right),
$$

as expected from the divisor relation [15, p527]. By the divisor relation, (1.7), (2.23), (2.24), and (2.3),

$$
\begin{aligned}
\langle\mathbf{a}\rangle+\sum_{d=1}^{\infty} Q^{d} d N_{d}^{X} X_{\mathbf{a}}\left(c_{1}, c_{2}\right) & =\langle\mathbf{a}\rangle \frac{I_{c_{1}+1}(q)}{I_{1}(q)} \quad \text { if } \quad c_{1}+c_{2}=n-2-l ; \\
\langle\mathbf{a}\rangle+\sum_{d=1}^{\infty} Q^{d} d^{2} N_{d}^{X_{\mathbf{a}}}(n-3-l) & =\langle\mathbf{a}\rangle \frac{I_{2}(q)}{I_{1}(q)} ;
\end{aligned}
$$

these identities are equations (1.5) and (1.6) in [24].

The first true cases of (1.7) and (1.8) occur for Calabi-Yau 6-folds and 7-folds:

$$
\left(n, \mathbf{a}, c_{1}, c_{2}, c_{3}\right)=(8,(8), 2,2,2) \quad \text { and } \quad\left(n, \mathbf{a}, c_{1}, c_{2}, c_{3}, c_{4}\right)=(9,(9), 2,2,2,2) .
$$

Tables 144 show some low-degree BPS counts obtained from (1.7) and (1.8) via [17, (2)] for all complete intersections $X_{\mathbf{a}} \subset \mathbb{P}^{n-1}$, with $n \leq 10$, of suitable dimensions, with $H^{c_{i}}$ indicating that one of the constraints is a general linear subspace of $\mathbb{P}^{n-1}$ of codimension $c_{i}$. All degree 1 and 2 numbers agree with the corresponding lines and conics counts obtained via classical Schubert calculus computations (the 3-pointed numbers for hypersurfaces can be found in [16], which also describes the classical methods). The degree 3 numbers for the hypersurfaces $X_{8}$ and $X_{9}$ agree with [8]; the remaining degree 3 numbers can presumably be confirmed by similar computations. The most noteworthy is the agreement of the 4-pointed numbers, since these do not naturally arise in the physics view of mirror symmetry as originally presented in [13. 4 There are currently no direct methods of counting curves of degree 4 or higher on projective complete intersections; so the numbers in these degrees obtained from (1.7) and (1.8) cannot be compared to anything at this time. Finally, all BPS counts computed from (1.7) and (1.8) via [17, (2)] for $d \leq 100$ and all compete intersections $X_{\mathbf{a}} \subset \mathbb{P}^{n-1}$ with $n \leq 10$ are integers, as the case should be 5

\footnotetext{
${ }^{4}$ This viewpoint is extended to arbitrary number of marked points in [4].

${ }^{5}$ The genus $0 \mathrm{GW}$-invariants of CYs with at least 3 marked points are integers; see [22, Section 7.3] and [25]. Since the GW-BPS transform of [17, (2)] is always lower-triangular with 1's on the diagonal and integers everywhere else if the number of marked points is at least 3, it follows that the BPS numbers are integers as well in this case.
} 


\begin{tabular}{c||c|c|c|c}
\hline$d$ & 1 & 2 & 3 & 4 \\
\hline$X_{8}$ & 59021312 & 821654025830400 & 12197109744970010814464 & 186083410628492378226388631552 \\
$X_{27}$ & 19133912 & 52069545843672 & 150771900962422866056 & 448721851648931529402358688 \\
$X_{36}$ & 9303984 & 9656915909184 & 10669913703022812624 & 12119013327306237518117376 \\
$X_{45}$ & 6536800 & 4306289363200 & 3019921285456823200 & 2177140100777199737600000 \\
$X_{226}$ & 7036416 & 4323279882240 & 2819049510852887040 & 1889305224389886741405696 \\
$X_{235}$ & 3936600 & 1091194853400 & 321105896368043400 & 97128823290992207460000 \\
$X_{244}$ & 3252224 & 699998060544 & 159942140236292096 & 37565431180080918822912 \\
$X_{334}$ & 2589408 & 396151430400 & 64359976334347296 & 10748812573405031454720 \\
\hline
\end{tabular}

Table 1: Low-degree genus 0 BPS numbers $\left(H^{2}, H^{2}, H^{2}\right)$ for some Calabi-Yau 6-folds

\begin{tabular}{c||c|c|c}
\hline$d$ & 1 & 2 & 3 \\
\hline$X_{9}$ & 1579510449 & 506855012110118424 & 174633921378662035929052320 \\
$X_{28}$ & 466477056 & 25865899481481216 & 1538349758855955308748800 \\
$X_{37}$ & 200848599 & 3684692607275358 & 72513809257771729565550 \\
$X_{46}$ & 122812416 & 1209608310822912 & 12780622639872867502080 \\
$X_{55}$ & 104480625 & 841277146035000 & 7266883194629367785000 \\
\hline
\end{tabular}

Table 2: Low-degree genus 0 BPS numbers $\left(H^{2}, H^{2}, H^{3}\right)$ for some Calabi-Yau 7-folds

\begin{tabular}{c||c|c|c}
\hline$d$ & 1 & 2 & 3 \\
\hline$X_{9}$ & 2395066806 & 1718927099008463268 & 957208127608222375829677128 \\
$X_{28}$ & 702562304 & 86939314932416512 & 8348345278919524413816832 \\
$X_{37}$ & 302321376 & 12364886269091538 & 392695531026064094763648 \\
$X_{46}$ & 184771584 & 4056318495977472 & 69156291871338627290112 \\
$X_{55}$ & 157178750 & 2820556380767500 & 39310596116635041745000 \\
\hline
\end{tabular}

Table 3: Low-degree genus 0 BPS numbers $\left(H^{2}, H^{2}, H^{2}, H^{2}\right)$ for some Calabi-Yau 7-folds

\begin{tabular}{c||c|c|c}
\hline$d$ & 1 & 2 & 3 \\
\hline$\left(H^{2}, H^{3}, H^{3}\right)$ & 51415320000 & 444475303469701680000 & 4089048226644406809222184680000 \\
$\left(H^{2}, H^{2}, H^{4}\right)$ & 38922224000 & 295035175517918176000 & 2467449594491156931046837776000 \\
$\left(H^{2}, H^{2}, H^{2}, H^{3}\right)$ & 75062592000 & 1394799570099498816000 & 20109980886063766606715932224000 \\
\hline
\end{tabular}

Table 4: Low-degree genus 0 BPS numbers for $X_{10}$ in $\mathbb{P}^{9}$

\subsection{The projective case}

Throughout the paper, we denote by $\overline{\mathbb{Z}}^{+}$the set of nonnegative integers. If $N, d, n \in \overline{\mathbb{Z}}^{+}$, let

$$
\begin{aligned}
& \mathcal{P}_{N}(d)=\left\{\mathbf{d} \equiv\left(d_{1}, d_{2}, \ldots, d_{N}\right) \in\left(\overline{\mathbb{Z}}^{+}\right)^{N}: \sum_{s=1}^{s=N} d_{s}=d\right\} \\
& \mathcal{P}_{N}^{n}(d)=\left\{\mathbf{d} \equiv\left(d_{1}, d_{2}, \ldots, d_{N}\right) \in \mathcal{P}_{N}(d): \quad d_{s}<n \forall s \in[N]\right\} .
\end{aligned}
$$


For any $\mathbf{p} \in \mathcal{P}_{N}^{n}(d)$, let

$$
(\mathbf{p})=\min \left\{p_{s}+1, n-1-p_{s}: s \in[N]\right\}
$$

If $\left(c_{s}\right)_{s \in[N]} \in\left(\overline{\mathbb{Z}}^{+}\right)^{N}$, let

$$
\left\langle\prod_{s=1}^{s=N} \frac{H^{c_{s}}}{\hbar_{s}-\psi}\right\rangle_{0, d}^{\mathbb{P}^{n-1}}=\sum_{b_{1}, b_{2}, \ldots, b_{N} \geq 0}\left(\prod_{s=1}^{s=N} \hbar_{s}^{-1-b_{s}}\right)\left\langle\tau_{b_{1}} H^{c_{1}}, \ldots, \tau_{b_{N}} H^{c_{N}}\right\rangle_{0, d}^{\mathbb{P}^{n-1}} .
$$

Theorem A yields fairly simple closed formulas for the genus $0 \mathrm{GW}$-invariants of projective spaces with 3 and 4 insertions. Theorem 4 below follows immediately from (2.1), (2.35), (2.33), (2.40), (2.44), (2.20), (2.18), (2.14), and (1.4) 6

Theorem 4. The 3-and 4-pointed Gromov-Witten invariants of $\mathbb{P}^{n-1}$ are described by

$$
\begin{gathered}
\sum_{p_{1}, p_{2}, p_{3} \geq 0}\left\langle\prod_{s=1}^{s=3} \frac{H^{n-1-p_{s}}}{\hbar_{s}-\psi}\right\rangle_{0, d}^{\mathbb{P}^{n-1}} H_{1}^{p_{1}} H_{2}^{p_{2}} H_{3}^{p_{3}}=\sum_{d^{\prime}=0}^{d^{\prime}=1} \sum_{\substack{\mathbf{d} \in \mathcal{P}_{3}\left(d-d^{\prime}\right) \\
\mathbf{p} \in \mathcal{P}_{3}^{n}\left(\left(2-d^{\prime}\right) n-2\right)}} \prod_{s=1}^{s=3} \frac{\left(H_{s}+d_{s} \hbar_{s}\right)^{p_{s}}}{\hbar_{s} \prod_{r=1}^{d_{s}}\left(H_{s}+r \hbar_{s}\right)^{n}}, \\
\sum_{p_{1}, p_{2}, p_{3}, p_{4} \geq 0}\left\langle\prod_{s=1}^{s=4} \frac{H^{n-1-p_{s}}}{\hbar_{s}-\psi}\right\rangle_{0, d}^{\mathbb{P}^{n-1}} H_{1}^{p_{1}} H_{2}^{p_{2}} H_{3}^{p_{3}} H_{4}^{p_{4}} \\
=\left\{\sum_{\substack{\mathbf{d} \in \mathcal{P}_{3}(d-1) \\
\mathbf{p} \in \mathcal{P}_{4}^{n}(2 n-4)}}(\mathbf{p})+\left(\sum_{s=1}^{s=4} \hbar_{s}^{-1}\right) \sum_{d^{\prime}=0}^{d^{\prime}=2} \sum_{\substack{\mathbf{d} \in \mathcal{P}_{4}\left(d-d^{\prime}\right) \\
\mathbf{p} \in \mathcal{P}_{4}^{n}\left(\left(3-d^{\prime}\right) n-3\right)}}^{s=4} \prod_{s=1}^{s} \frac{\left(H_{s}+d_{s} \hbar_{s}\right)^{p_{s}}}{\hbar_{s} \prod_{s}\left(H_{s}+r \hbar_{s}\right)^{n}}\right.
\end{gathered}
$$

both identities hold modulo $H_{s}^{n}$ and as power series in $\hbar_{s}^{-1}$.

Since the $d=1$ Gromov-Witten invariant counts lines in $\mathbb{P}^{n-1}$, the $d=1$ case of the 4 -pointed formula in Theorem 4 gives

$$
\left\langle\sigma_{c_{1}} \sigma_{c_{2}} \sigma_{c_{3}} \sigma_{c_{4}}, \mathbb{G}(2, n)\right\rangle=\min \left\{c_{s}+1, n-1-c_{s}: s=1,2,3,4\right\} \quad \text { if } c_{s} \in \overline{\mathbb{Z}}^{+}, \sum_{s=1}^{s=4} c_{s}=2 n-4,
$$

where $\sigma_{c}$ is the usual codimension $c$ Schubert cycle on $\mathbb{G}(2, n)$. As pointed out to the author by A. Buch, this identity can be confirmed by applying Pieri's rule [14, p203] to $\sigma_{c_{1}} \sigma_{c_{2}}$ and $\sigma_{c_{3}} \sigma_{c_{4}}$ and counting pairs of dual cycles in its outputs. The $d=2$ case of the 4 -pointed formula gives

$$
\left\langle H^{c_{1}}, H^{c_{2}}, H^{c_{3}}, H^{c_{4}}\right\rangle_{0,2}^{\mathbb{P}^{n-1}}=0 .
$$

This is indeed as expected, since every conic lies in a $\mathbb{P}^{2}\left[14\right.$, p177] and no $\mathbb{P}^{2}$ meets general linear subspaces of $\mathbb{P}^{n-1}$ of total codimension $3 n$ (the space of planes meeting the constraints is the intersection of Schubert cycles in $\mathbb{G}(3, n)$ of total codimension $3 n-8$ and is thus empty).

\section{Main Theorem}

In addition to the notation introduced at the beginning of Section 1.2 , for any $m, l \in \overline{\mathbb{Z}}^{+}$we define

$$
\llbracket m \rrbracket=\left\{s \in \overline{\mathbb{Z}}^{+}: s<m\right\}, \quad \llbracket m \rrbracket_{l}=\{s \in \llbracket m \rrbracket: s \geq l\} .
$$

\footnotetext{
${ }^{6}$ in this case, $\tilde{\mathrm{c}}_{p, s}^{(d)}=\delta_{0, d} \delta_{p, s}$ in (2.18) and (2.40); (2.44) is needed for the second identity in Theorem 4 only
} 
We denote by $\mathcal{P}_{m}([N])$ the set of unordered partitions $\mathbf{S} \equiv\left\{S_{i}\right\}_{i \in[m]}$ of $[N]$ into nonempty subsets $S_{i}$ such that one of them is $\{N\}, 7$ If $\mathbf{p}$ is an $N$-tuple of integers, $S \subset[N]$, and $p^{\prime} \in \mathbb{Z}$, let $\left.\mathbf{p}\right|_{S}$ and $\mathbf{p} p^{\prime}$ denote the $S$-tuple consisting of the elements of $\mathbf{p}$ indexed by $S$ and the $(N+1)$-tuple obtained by adjoining $p^{\prime}$ to $\mathbf{p}$ at the end, respectively, and set

$$
|\mathbf{p}|_{S} \equiv|\mathbf{p}|_{S} \mid \equiv \sum_{s \in S} p_{s}
$$

If $R$ is a ring and $\underline{x}=\left(x_{1}, \ldots, x_{N}\right)$ is a tuple of variables, let

$$
R[\underline{x}]=R\left[x_{1}, \ldots, x_{N}\right]
$$

be the ring of polynomials in $x_{1}, \ldots, x_{N}$. If $\Phi \in R[[q]]$ and $d \in \mathbb{Z}$, let $\llbracket \Phi \rrbracket_{q ; d} \in R$ denote the coefficient of $q^{d}\left(\llbracket \Phi \rrbracket_{q ; d} \equiv 0\right.$ if $\left.d<0\right)$.

Let $\mathbb{P}_{N}^{n-1}=\left(\mathbb{P}^{n-1}\right)^{N}$. For each $s=1, \ldots, N$, we set

$$
H_{s}=\pi_{s}^{*} H \in H^{2}\left(\mathbb{P}_{N}^{n-1}\right),
$$

where $\pi_{s}: \mathbb{P}_{N}^{n-1} \longrightarrow \mathbb{P}^{n-1}$ is the projection onto the $s$-th coordinate. Since $\overline{\mathfrak{M}}_{0, N}\left(\mathbb{P}^{n-1}, d\right)$ is smooth, there is a well-defined cohomology push-forward

$$
\mathrm{ev}_{*} \equiv\left\{\mathrm{ev}_{1} \times \ldots \mathrm{ev}_{N}\right\}_{*}: H^{*}\left(\overline{\mathfrak{M}}_{0, N}\left(\mathbb{P}^{n-1}, d\right)\right) \longrightarrow H^{*}\left(\mathbb{P}_{N}^{n-1}\right)
$$

With $\underline{\hbar}=\left(\hbar_{1}, \ldots, \hbar_{N}\right), \underline{\hbar}^{-1}=\left(\hbar_{1}^{-1}, \ldots, \hbar_{N}^{-1}\right)$, and $\underline{H}=\left(H_{1}, \ldots, H_{N}\right)$, let

$$
Z(\underline{\hbar}, \underline{H}, Q)=\sum_{d=0}^{\infty} Q^{d} \mathrm{ev}_{*}\left\{\frac{e\left(\mathcal{V}_{d}\right)}{\prod_{s=1}^{s=N}\left(\hbar_{s}-\psi_{s}\right)}\right\} \in H^{*}\left(\mathbb{P}_{N}^{n-1}\right)\left[\underline{\hbar}^{-1}\right][[Q]] .
$$

By (1.3), this power series encodes all genus $0 \mathrm{GW}$-invariants of $X_{\mathbf{a}}$ with constrains that arise from $\mathbb{P}^{n-1}$. If $\mathbf{b} \equiv\left(b_{1}, \ldots, b_{N}\right) \in \mathbb{Z}^{N}$, let

$$
\underline{\hbar}^{-\mathbf{b}}=\prod_{s=1}^{s=N}\left(\hbar_{s}^{-1}\right)^{b_{s}} .
$$

\subsection{An asymptotic expansion}

The power series $F$ defined by (1.4) admits an asymptotic expansion $w \longrightarrow \infty$ which plays a central role in this paper and which we now describe.

Define

$$
\begin{array}{ll}
L(q) \in 1+q \mathbb{Q}[[q]] & \text { by } \quad L(q)^{n}-\mathbf{a}^{\mathbf{a}} q L(q)^{|\mathbf{a}|}=1 \in \mathbb{Q}[[q]], \\
\chi_{0}, \chi_{1}, \ldots, \chi_{|\mathbf{a}|} \in \mathbb{Q} & \text { by } \quad \prod_{k=1}^{k=l} \prod_{r=1}^{r=a_{k}}\left(a_{k} D+r\right) \equiv \mathbf{a}^{\mathbf{a}} \sum_{i=0}^{i=|\mathbf{a}|} \chi_{|\mathbf{a}|-i} D^{i} \in \mathbb{Z}[D] .
\end{array}
$$

\footnotetext{
${ }^{7}$ More precisely, $\mathcal{P}_{m}([N])$ consists of unordered partitions with a choice of some ordering for each of the partitions.
} 
In the two extremal cases, (2.2) gives

$$
L(q)= \begin{cases}(1+q)^{1 / n}, & \text { if }|\mathbf{a}|=0 \\ \left(1-\mathbf{a}^{\mathbf{a}} q\right)^{-1 / n}, & \text { if }|\mathbf{a}|=n .\end{cases}
$$

Setting $\chi_{i} \equiv 0$ if $i<0$ or $i>|\mathbf{a}|$, we find that

$$
\chi_{0}=1, \quad \chi_{1}=\frac{|\mathbf{a}|+l}{2} .
$$

For $m, j \in \mathbb{Z}$, we define $\mathcal{H}_{m, j} \in \mathbb{Q}(u)$ recursively by

$$
\begin{aligned}
\mathcal{H}_{m, j} & \equiv 0 \quad \text { unless } 0 \leq j \leq m, \quad \mathcal{H}_{0,0} \equiv 1 ; \\
\mathcal{H}_{m, j}(u) & \equiv \mathcal{H}_{m-1, j}(u)+\frac{u-1}{|\mathbf{a}|+\nu_{\mathbf{a}} u}\left(n u \frac{\mathrm{d}}{\mathrm{d} u}+m-j\right) \mathcal{H}_{m-1, j-1}(u) \quad \text { if } m \geq 1,0 \leq j \leq m .
\end{aligned}
$$

In particular, for $m \geq 0$

$$
\mathcal{H}_{m, 0}(u)=1, \quad \mathcal{H}_{m, 1}(u)=\left(\begin{array}{c}
m \\
2
\end{array}\right) \frac{u-1}{|\mathbf{a}|+\nu_{\mathbf{a}} u} .
$$

Finally, we define differential operators $\mathfrak{L}_{1}, \ldots, \mathfrak{L}_{n}$ on $\mathbb{Q}[[q]]$ by

$$
\mathfrak{L}_{k}=\sum_{i=0}^{k}\left[\left(\begin{array}{c}
n \\
i
\end{array}\right) L^{n} \mathcal{H}_{n-i, k-i}\left(L^{n}\right)-\left(L^{n}-1\right) \sum_{r=0}^{k-i}\left(\begin{array}{c}
|\mathbf{a}|-r \\
i
\end{array}\right) \chi_{r} \mathcal{H}_{|\mathbf{a}|-i-r, k-i-r}\left(L^{n}\right)\right] D^{i},
$$

where $D=q \frac{\mathrm{d}}{\mathrm{d} q}$. By (2.6), (2.4) and (2.2), the first of these operator is

$$
\begin{aligned}
\mathfrak{L}_{1} & =\left(|\mathbf{a}|+\nu_{\mathbf{a}} L^{n}\right)\left\{D+\frac{L^{n}-1}{|\mathbf{a}|+\nu_{\mathbf{a}} L^{n}}\left(\frac{\nu_{\mathbf{a}} n L^{n}}{2\left(|\mathbf{a}|+\nu_{\mathbf{a}} L^{n}\right)}-\frac{l+1}{2}\right)\right\} \\
& =\left(|\mathbf{a}|+\nu_{\mathbf{a}} L^{n}\right)\left\{\left(\left(\frac{n}{|\mathbf{a}|+\nu_{\mathbf{a}} L^{n}}\right)^{1 / 2} L^{\frac{l+1}{2}}\right) D\left(\left(\frac{n}{|\mathbf{a}|+\nu_{\mathbf{a}} L^{n}}\right)^{1 / 2} L^{\frac{l+1}{2}}\right)^{-1}\right\} .
\end{aligned}
$$

Proposition 2.1. The power series $F$ of (1.4) admits an asymptotic expansion

$$
F(w, q) \sim e^{\xi(q) w} \sum_{b=0}^{\infty} \Phi_{b}(q) w^{-b} \quad \text { as } \quad w \longrightarrow \infty
$$

with $\xi, \Phi_{1}, \ldots \in q \mathbb{Q}[[q]]$ and $\Phi_{0} \in 1+q \mathbb{Q}[[q]]$ determined by the first-order ODEs

$$
1+\xi^{\prime}(q)=L(q), \quad \mathfrak{L}_{1} \Phi_{b}+\frac{1}{L} \mathfrak{L}_{2} \Phi_{b-1}+\ldots+\frac{1}{L^{n-1}} \mathfrak{L}_{n} \Phi_{b+1-n}=0,
$$

where $\Phi_{b} \equiv 0$ for $s<0$.

From (2.8) and (2.10), we immediately find that

$$
\Phi_{0}(q)=\left(\frac{n}{|\mathbf{a}|+\nu_{\mathbf{a}} L(q)^{n}}\right)^{1 / 2} L(q)^{(l+1) / 2} .
$$

In the extremal cases, this reduces to

$$
\Phi_{0}(q)= \begin{cases}L(q)^{-(n-1) / 2}=(1+q)^{-(n-1) / 2 n}, & \text { if }|\mathbf{a}|=0 \\ L(q)^{(l+1) / 2}=\left(1-\mathbf{a}^{\mathbf{a}} q\right)^{-(l+1) / 2 n}, & \text { if }|\mathbf{a}|=n .\end{cases}
$$

Proposition 2.1 in the $|\mathbf{a}|=n$ case is proved in [23, Section 4], building up on the $\mathbf{a}=(n)$ case contained in Lemma 1.3 and Theorems 1.1, 1.2, and 1.4 in [26]. The remaining cases are addressed in Appendix $\mathrm{A}$ 


\subsection{One- and two-pointed formulas}

By the dilaton relation [15, p527] and [10, Theorems 9.5,10.7,11.8], the generating function (2.1) with $N=1$ and the degree 0 term defined to be $\langle\mathbf{a}\rangle H_{1}^{l} \hbar_{1}$ is given by

$$
Z\left(\hbar_{1}, H_{1}, Q\right)=\langle\mathbf{a}\rangle H_{1}^{l} e^{-J\left(q_{1}\right) w_{1}} \hbar_{1} \frac{F\left(w_{1}, q_{1}\right)}{I_{0}\left(q_{1}\right)}, \quad \text { where } \quad w_{1}=\frac{H_{1}}{\hbar_{1}}, \quad q_{1} e^{\delta_{0 \nu_{\mathbf{a}}} J\left(q_{1}\right)}=\frac{Q}{H_{1}^{\nu_{\mathbf{a}}}} .
$$

The generating function (2.1) for $N=2$ is given in [24, Section 2] in terms of certain transforms of $F$, which we describe next.

Define

$$
\begin{gathered}
\mathbf{D}: \mathbb{Q}(w)[[q]] \longrightarrow \mathbb{Q}(w)[[q]] \quad \text { by } \quad \mathbf{D} H(w, q) \equiv\left\{1+\frac{q}{w} \frac{\mathrm{d}}{\mathrm{d} q}\right\} H(w, q) ; \\
F_{0}(w, q) \equiv \sum_{d=0}^{\infty} q^{d} w^{\nu_{\mathbf{a}}} \frac{\prod_{k=1}^{k=l} \prod_{r=0}^{a_{k} d-1}\left(a_{k} w+r\right)}{\prod_{r=1}^{d}\left((w+r)^{n}-w^{n}\right)} \in \mathcal{P} ; \\
F_{p} \equiv \mathbf{D}^{p} F_{0}=\mathbf{M}^{p} F_{0} \in \mathcal{P} \quad \forall p=1,2, \ldots, l .
\end{gathered}
$$

In particular, $F_{l}=F$. For $\nu_{\mathbf{a}}>0$, we also define $\mathrm{c}_{p, s}^{(d)}, \tilde{\mathrm{c}}_{l+p, l+s}^{(d)} \in \mathbb{Q}$ with $p, s, d \geq 0$ by

$$
\begin{gathered}
\sum_{d=0}^{\infty} \sum_{s=0}^{\infty} \mathrm{c}_{p, s}^{(d)} w^{s} q^{d}=\sum_{d=0}^{\infty} q^{d} \frac{(w+d)^{p} \prod_{k=1}^{l} \prod_{r=1}^{a_{k} d}\left(a_{k} w+r\right)}{\prod_{r=1}^{d}(w+r)^{n}}=w^{p} \mathbf{D}^{p} F\left(w, q / w^{\nu_{\mathbf{a}}}\right), \\
\sum_{\substack{d_{1}+d_{2}=d \\
d_{1}, d_{2} \geq 0}} \sum_{r=0}^{p-\nu_{\mathbf{a}} d_{1}} \tilde{\mathrm{c}}_{l+p, l+r}^{\left(d_{1}\right)} \mathrm{c}_{r, s}^{\left(d_{2}\right)}=\delta_{0, d} \delta_{p, s} \quad \forall d, s \in \overline{\mathbb{Z}}^{+}, s \leq p-\nu_{\mathbf{a}} d .
\end{gathered}
$$

Since $c_{p, s}^{(0)}=\delta_{p, s}$, the second equation in (2.17) expresses $\tilde{\mathrm{c}}_{l+p, l+s}^{(d)}$ with $s \leq p-\nu_{\mathbf{a}} d$ in terms of the numbers $\tilde{\mathrm{c}}_{l+p, l+r}^{\left(d_{1}\right)}$ with $d_{1}<d$; the numbers $\tilde{\mathrm{c}}_{l+p, l+s}^{(d)}$ with $s>p-\nu_{\mathbf{a}} d$ will not be needed. In particular, $\tilde{\mathrm{c}}_{p, s}^{(0)}=\delta_{p, s}$ for all $p, s \geq l$. For $p>l$, set

$$
F_{p}(w, q)= \begin{cases}\mathbf{M}^{p} F(w, q), & \text { if } \nu_{\mathbf{a}}=0 \\ \sum_{d=0}^{\infty} \sum_{s=0}^{p-l-\nu_{\mathbf{a}} d} \frac{\tilde{c}_{p, l+s}^{(d)} q^{d}}{w^{p-l-\nu_{\mathbf{a}} d-s}} \mathbf{D}^{s} F(w, q), & \text { if } \nu_{\mathbf{a}}>0\end{cases}
$$

Thus, $F_{p} \in \mathcal{P}$ for all $p \in \mathbb{Z}^{+}$by (2.17) and $F_{p}=\mathbf{D}^{p-l} F$ unless $p \geq l+\nu_{\mathbf{a}}$. By [24, Theorem 3], the generating function (2.1) with $N=2$ and the degree 0 term defined to be the image of $\frac{\langle\mathbf{a}\rangle H_{1}^{l} H_{2}^{l}}{\hbar_{1}+\hbar_{2}} \frac{H_{1}^{n-l}-H_{2}^{n-l}}{H_{1}-H_{2}}$ is given by

$$
Z\left(\hbar_{1}, \hbar_{2}, H_{1}, H_{2}, Q\right)=\frac{\langle\mathbf{a}\rangle}{\hbar_{1}+\hbar_{2}} e^{-J\left(q_{1}\right) w_{1}-J\left(q_{2}\right) w_{2}} \sum_{\substack{p_{1}+p_{2}=n-1+l \\ p_{1}, p_{2} \geq l}} \prod_{\substack{s=1 \\ s=2}}^{s} H_{s}^{p_{s}} \frac{F_{p_{s}}\left(w_{s}, q_{s}\right)}{I_{p_{s}-l}(q)}
$$


where

$$
w_{s}=\frac{H_{s}}{\hbar_{s}}, \quad q_{s} e^{\delta_{0 \nu_{\mathbf{a}}} J\left(q_{s}\right)}=\frac{Q}{H_{s}^{\nu_{\mathbf{a}}}} .
$$

Remark 2.2. The mismatch in the indexing of $I_{*}$ and $F_{*}$ is unfortunate for the purposes of this section. However, the choice of the indexing for the former is intended to simplify the explicit formulas for the Calabi-Yau CIs in Section 1.1, while the choice of the indexing for the latter is intended to simplify some of the formulas in the proof of Theorem $\mathrm{A}$ in the rest of the paper.

\subsection{Multi-pointed formulas}

Similarly to (2.19), the generating function (2.1) with $N \geq 3$ is a linear combination of the $N$-fold products

$$
\Delta_{\mathbf{p}}(\underline{\hbar}, \underline{H}, Q) \equiv \prod_{s=1}^{s=N} \frac{H^{p_{s}}}{\hbar_{s}} \frac{F_{p_{s}}\left(w_{s}, q_{s}\right)}{\prod_{\substack{n-l-1 \\ n-p_{s}-l}} I_{r}\left(q_{s}\right)}, \quad \text { where } \quad w_{s}=\frac{H_{s}}{\hbar_{s}}, \quad q_{s} e^{\delta_{0 \nu_{\mathbf{a}}} J\left(q_{s}\right)}=\frac{Q}{H_{s}^{\nu_{\mathbf{a}}}}
$$

with $\mathbf{p}=\left(p_{1}, p_{2}, \ldots, p_{N}\right) \in \llbracket n \rrbracket^{N}, p_{s} \geq l$, and with coefficients that are polynomials in $\hbar_{1}^{-1}, \ldots, \hbar_{N}^{-1}$ of total degree at most $N-3$. These coefficients are described below inductively using the coefficients $\tilde{\mathrm{c}}_{p, s}^{(d)}$ defined above and the asymptotic expansion of $F(w, q)$ provided by Proposition 2.1 .

For $r<0$, we set $I_{r}(q)=1$. By Proposition 2.1, (2.14)-(2.16), and (2.18), there are asymptotic expansions

$$
\frac{F_{p}(w, p)}{n-l-1} \sim e^{\xi(q) w} \frac{I_{0}(q)}{L(q)^{\delta \nu_{\mathbf{a}}} n} \sum_{b=0}^{\infty} \Phi_{p ; b}(q) w^{-b} \quad \text { as } \quad w \longrightarrow \infty,
$$

with $\Phi_{p ; 0} \in 1+q \mathbb{Q}[[q]]$ and $\Phi_{p ; 1}, \Phi_{p ; 2} \ldots \in q \mathbb{Q}[[q]]$ described by

$$
\begin{gathered}
\hat{\Phi}_{p+1 ; b}=L \hat{\Phi}_{p ; b}+\hat{\Phi}_{p ; b-1}^{\prime}-\left(\sum_{r=0}^{r=p} \frac{I_{r}^{\prime}}{I_{r}}\right) \hat{\Phi}_{p ; b-1} \quad \forall p \in \mathbb{Z}, \quad \hat{\Phi}_{0 ; b}=\Phi_{b}, \\
\Phi_{p ; b}(q)= \begin{cases}\sum_{d=0}^{\infty} \sum_{s=0}^{p-\nu_{\mathbf{a}} d} \tilde{\mathrm{c}}_{p, s}^{(d)} q^{d} \hat{\Phi}_{s-l ; b-\left(p-\nu_{\mathbf{a}} d-s\right)}(q), & \text { if } \nu_{\mathbf{a}}>0, \\
\hat{\Phi}_{p-l ; b}(q), & \text { if } \nu_{\mathbf{a}}=0,\end{cases}
\end{gathered}
$$

where $\hat{\Phi}_{p ; b} \equiv 0$ if $b<0, \tilde{\mathrm{c}}_{p, s}^{(d)} \equiv \delta_{0, d} \delta_{p, s}$ unless $p, s \geq l$, and ' denotes $q \frac{\mathrm{d}}{\mathrm{d} q}$ as before. In the Calabi-Yau case, $\nu_{\mathbf{a}}=0$, the recursion (2.22) for the coefficients $\Phi_{p ; b}=\hat{\Phi}_{p-l ; b}$ in the asymptotic expansion (2.21) is obtained using the first two identities in the following lemma 8

Lemma 2.3 ([23, Proposition 4.4]). If $|\mathbf{a}|=n$, the power series $I_{p}$ defined by (1.4) and (1.5) satisfy

$$
\begin{aligned}
I_{n-l-p} & =I_{p} \quad \forall p=0,1, \ldots, n-l ; \\
I_{0} I_{1} \cdots I_{n-l} & =L^{n} ; \\
I_{0}^{n-l} I_{1}^{n-l-1} \cdots I_{n-l}^{0} & =L^{\frac{n(n-l)}{2}} .
\end{aligned}
$$

\footnotetext{
${ }^{8}$ The last identity in Lemma 2.3 follows from the first two; it was used in Section 1.1
} 
For example, by (2.22),

$$
\begin{aligned}
& \hat{\Phi}_{p ; 0}=L^{p} \Phi_{0}, \quad \hat{\Phi}_{p ; 1}=L^{p}\left(\Phi_{1}+\mathbb{A}_{p}^{(1)} \Phi_{0}\right) ; \quad \frac{\Phi_{p ; 0}(q)}{\Phi_{0}(q)}=L(q)^{p-l} \begin{cases}\sum_{d=0}^{\infty} \frac{\tilde{c}_{p, p-\nu_{\mathbf{a}} q^{d}}^{(d)}}{L(q)^{\nu_{\mathbf{a}} d}}, & \text { if } \nu_{\mathbf{a}}>0 ; \\
1, & \text { if } \nu_{\mathbf{a}}=0 ;\end{cases} \\
& \frac{\Phi_{p ; 1}(q)}{\Phi_{0}(q)}=L(q)^{p-l} \begin{cases}\sum_{d=0}^{\infty} \frac{\tilde{c}_{p, p-\nu_{\mathbf{a}} d}^{(d)} q^{d}}{L(q)^{\mathbf{2}} d}\left(\frac{\Phi_{1}(q)}{\Phi_{0}(q)}+\mathbb{A}_{p-l-\nu_{\mathbf{a}} d}^{(1)}(q)\right)+\sum_{d=0}^{\infty} \frac{\tilde{c}_{p, p-\nu_{\mathbf{a}} d-1}^{(d)} q^{d}}{L(q)^{\mathbf{a}} d+1}, & \text { if } \nu_{\mathbf{a}}>0, \\
\frac{\Phi_{1}(q)}{\Phi_{0}(q)}+\mathbb{A}_{p-l}^{(1)}(q), & \text { if } \nu_{\mathbf{a}}=0,\end{cases}
\end{aligned}
$$

where

$$
\tilde{\mathrm{c}}_{p, s}^{(d)} \equiv 0 \quad \text { if } s+\nu_{\mathbf{a}} d>p, \quad \mathbb{A}_{p}^{(1)}=L^{-1}\left(p \frac{\Phi_{0}^{\prime}}{\Phi_{0}}+\frac{p(p-1)}{2} \frac{L^{\prime}}{L}-\sum_{r=0}^{r=p}(p-r) \frac{I_{r}^{\prime}}{I_{r}}\right) .
$$

In the two extremal cases, (2.12) gives

$$
\mathbb{A}_{p}^{(1)}=L^{-1} \begin{cases}-\frac{(n-p) p}{2} \frac{L^{\prime}}{L}, & \text { if }|\mathbf{a}|=0 \\ \frac{(p+l) p}{2} \frac{L^{\prime}}{L}-\sum_{r=0}^{r=p}(p-r) \frac{I_{r}^{\prime}}{I_{r}}, & \text { if }|\mathbf{a}|=n\end{cases}
$$

If $m \in \overline{\mathbb{Z}}^{+}, d, t \in \mathbb{Z}$, and $\mathbf{c} \equiv\left(c_{r}\right)_{r \in \mathbb{Z}^{+}} \in\left(\overline{\mathbb{Z}}^{+}\right)^{\infty}$, let

$$
\begin{aligned}
\mathcal{S}_{m}(d, t) & =\left\{(\mathbf{p}, \mathbf{b}) \in\left\lfloor n \rrbracket^{m} \times \mathbb{Z}^{m}:|\mathbf{p}|-|\mathbf{b}|=n-2+(m-1)(l+2)+\nu_{\mathbf{a}} d+n t\right\},\right. \\
\Phi_{m, \mathbf{c}} & =\frac{\Phi_{0}^{2}}{I_{0}^{2}}(-1)^{m+|\mathbf{c}|}(m+|\mathbf{c}|) ! \prod_{r=1}^{\infty} \frac{1}{c_{r} !}\left(\frac{\Phi_{r}}{(r+1) ! \Phi_{0}}\right)^{c_{r}} .
\end{aligned}
$$

For any $p, p^{\prime} \in\left\lfloor n \rrbracket\right.$ and $b, b^{\prime}, d, t \in \mathbb{Z}$, let

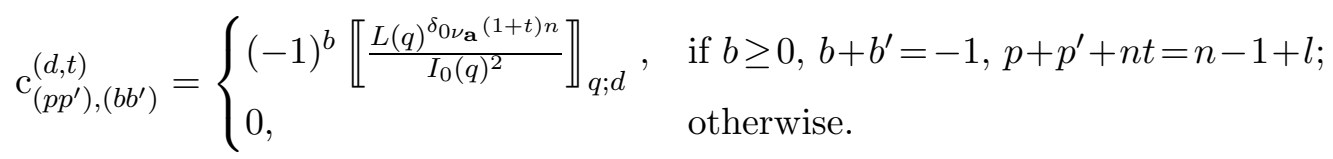

For any $N$-tuples $\mathbf{p} \in \llbracket n \rrbracket^{N}, \mathbf{b} \in\left(\overline{\mathbb{Z}}^{+}\right)^{N}$ with $N \geq 3$ and $d, t \in \overline{\mathbb{Z}}^{+}$, we inductively define

$$
\begin{aligned}
& \mathrm{c}_{\mathbf{p}, \mathbf{b}}^{(d, t)}=\sum_{\substack{m, d^{\prime}, t^{\prime} \in \mathbb{Z} \\
m \geq 3}} \sum_{\substack{\mathbf{S} \in \mathcal{P}_{m}([N]) \\
\mathbf{d} \in \mathcal{P}_{m}\left(d-d^{\prime}\right) \\
\mathbf{t} \in \mathcal{P}_{m}\left(t-t^{\prime}\right) \\
\left(\mathbf{p}^{\prime}, \mathbf{b}^{\prime}\right) \in \mathcal{S}_{m}\left(d^{\prime}, t^{\prime}\right)}} \sum_{\substack{\left|\mathbf{b}^{\prime \prime}\right|+\|\mathbf{c}\|\left(\mathbb{\mathbb { Z }}^{+}\right)^{m} \\
\mathbf{\mathbf { w } ^ { \prime }} \in m-3}}\left(\left(\prod_{i=1}^{i=m} \mathrm{c}_{\left.\mathbf{p}\right|_{S_{i}} p_{i}^{\prime},\left.\mathbf{b}\right|_{S_{i}} b_{i}^{\prime}}^{\left(d_{i}, t_{i}\right)}\right)\right. \\
& \left.\times \llbracket \Phi_{m-3, \mathbf{c}}(q) \prod_{i=1}^{i=m} \frac{I_{0}(q)^{2} \Phi_{p_{i}^{\prime} ; b_{i}^{\prime}+1+b_{i}^{\prime \prime}}(q)}{b_{i}^{\prime \prime} ! L(q)^{\delta_{0 \nu_{\mathbf{a}}} n} \Phi_{0}(q)} \rrbracket_{q ; d^{\prime}}\right),
\end{aligned}
$$

where $\Phi_{p ; b} \equiv 0$ if $b<0$ and $\mathrm{c}_{\mathbf{p}\left|S_{i} p_{i}^{\prime}, \mathbf{b}\right|_{S_{i}} b_{i}^{\prime}}^{\left(d_{i}, t_{i}\right)} \equiv 0$ if $b_{i}^{\prime}<0$ and $\left|S_{i}\right| \geq 2$. By induction,

$$
\mathrm{c}_{\mathbf{p}, \mathbf{b}}^{(d, t)} \neq 0 \quad \Longrightarrow \quad|\mathbf{b}| \leq N-3, \quad|\mathbf{p}|-|\mathbf{b}|+\nu_{\mathbf{a}} d+n t=(N-1)(n-2)+2+l .
$$

Since $\Phi_{m-3, \mathbf{c}}, \Phi_{p_{i}^{\prime} ; b_{i}^{\prime}+1+b_{i}^{\prime \prime}} \in q \mathbb{Q}[[q]]$ unless $\mathbf{c}=\mathbf{0}$ and $b_{i}^{\prime}+1+b_{i}^{\prime \prime}=0$,

$$
\mathrm{c}_{\mathbf{p}, \mathbf{b}}^{(0, t)}=\delta_{|\mathbf{p}|+n t,(N-1)(n-1)+l}\left(\begin{array}{c}
N-3 \\
\mathbf{b}
\end{array}\right) .
$$


Theorem A. Suppose $n, N \in \mathbb{Z}^{+}$, with $N \geq 3$, and $\mathbf{a} \in\left(\mathbb{Z}^{+}\right)^{l}$ is such that $\|\mathbf{a}\| \leq n$. The generating function (2.1) for $N$-pointed genus $0 G W$-invariants of a complete intersection $X_{\mathbf{a}} \subset \mathbb{P}^{n-1}$ is given by

$$
Z(\underline{\hbar}, \underline{H}, Q)=\langle\mathbf{a}\rangle e^{-\sum_{s=1}^{s=N} J\left(q_{s}\right) w_{s}} \sum_{\mathbf{p} \in \llbracket n \|_{l}^{N}} \sum_{\mathbf{b} \in\left(\overline{\mathbb{Z}}^{+}\right)^{N}} \sum_{d=0}^{\infty} \mathrm{c}_{\mathbf{p}, \mathbf{b}}^{(d, 0)} q^{d} \underline{\hbar}^{-\mathbf{b}} \Delta_{\mathbf{p}}(\underline{\hbar}, \underline{H}, Q),
$$

where $w_{s}=H_{s} / \hbar_{s}, q_{s} e^{\delta_{0 \nu_{\mathbf{a}} J\left(q_{s}\right)}}=Q / H_{s}^{\nu_{\mathbf{a}}}$, and $q e^{\delta_{0 \nu_{\mathbf{a}} J(q)}}=Q$.

We show in Section 3 that this theorem follows from Theorem B.

By (1.3), (2.1), (2.35), (2.34), and (2.20)

$$
\left\langle\tau_{b_{1}}\left(H^{c_{1}}\right), \ldots, \tau_{b_{N}}\left(H^{c_{N}}\right)\right\rangle_{0,0}^{X_{\mathbf{a}}}=\delta_{|\mathbf{c}|, n-1-l}\langle\mathbf{a}\rangle\left(\begin{array}{c}
N-3 \\
\mathbf{b}
\end{array}\right)
$$

whenever $b_{i}, c_{i} \geq 0$, as expected.

By (2.31), for each $p \in \llbracket n \rrbracket$, there exists a unique pair $\left(\hat{p}, t_{p}\right) \in \llbracket n \rrbracket \times \mathbb{Z}$ such that $\mathrm{c}_{(p \hat{p}),\left(b b^{\prime}\right)}^{\left(d, t_{p}\right)} \neq 0$ at least for some $b, b^{\prime}, d \in \mathbb{Z}$ :

$$
\left(\hat{p}, t_{p}\right)= \begin{cases}(n-1+l-p, 0), & \text { if } p \geq l \\ (l-1-p, 1), & \text { if } p<l\end{cases}
$$

For any $\mathbf{p} \in\left\lfloor n \rrbracket^{N}\right.$, let

$$
t_{\mathbf{p}}=\sum_{s=1}^{s=N} t_{p_{s}}=\left|\left\{s \in[N]: p_{s}<l\right\}\right|
$$

We note that

$$
\tilde{\mathrm{c}}_{\hat{p}, \hat{p}-\nu_{\mathbf{a}} d}^{(d)} \neq 0 \quad \Longrightarrow \quad p+\nu_{\mathbf{a}} d+(n-l) t_{p} \leq n-1
$$

If $N \geq 3, \mathbf{p} \in \llbracket n \rrbracket^{N}, \mathbf{b} \in\left(\overline{\mathbb{Z}}^{+}\right)^{N}, d \in \overline{\mathbb{Z}}^{+}$, and $t \in \mathbb{Z}$ satisfy the last property in (2.33) and $|\mathbf{b}|=N-3$, the only nonzero terms in (2.32) arise from $(m, \mathbf{c})=(N, \mathbf{0}), p_{i}^{\prime}=\hat{p}_{i}, b_{i}^{\prime}=-1-b_{i}$, and $b_{i}^{\prime \prime}=b_{i}$. If in addition $\nu_{\mathbf{a}} \neq 0$, by the last statement in (2.26), (2.11), and Lemma B.4

$$
\begin{aligned}
\mathbf{c}_{\mathbf{p}, \mathbf{b}}^{(d, t)} & =\left(\begin{array}{c}
N-3 \\
\mathbf{b}
\end{array}\right) \sum_{d^{\prime}=0}^{d^{\prime}=d} \tilde{\mathrm{c}}_{\hat{\mathbf{p}}}^{\left(d-d^{\prime}\right)} \llbracket \frac{n L(q)^{\nu_{\mathbf{a}} d^{\prime}+n\left(1+t-t_{\mathbf{p}}\right)}}{|\mathbf{a}|+\nu_{\mathbf{a}} L(q)^{n}} \|_{q ; d^{\prime}} \\
& =\left(\begin{array}{c}
N-3 \\
\mathbf{b}
\end{array}\right) \sum_{d^{\prime}=0}^{d^{\prime}=d}\left(\mathbf{a}^{\mathbf{a}}\right)^{d^{\prime}}\left(\begin{array}{c}
d^{\prime}+t-t_{\mathbf{p}} \\
d^{\prime}
\end{array}\right) \tilde{\mathrm{c}}_{\hat{\mathbf{p}}}^{\left(d-d^{\prime}\right)},
\end{aligned}
$$

with the binomial coefficients defined as in (B.5) and

$$
\tilde{\mathrm{c}}_{\hat{\mathbf{p}}}^{(d)} \equiv \sum_{\mathbf{d} \in \mathcal{P}_{N}(d)} \tilde{\mathrm{c}}_{\hat{\mathbf{p}}}^{(\mathbf{d})}, \quad \tilde{\mathrm{c}}_{\hat{\mathbf{p}}}^{(\mathbf{d})} \equiv \prod_{s=1}^{s=N} \tilde{\mathrm{c}}_{\hat{p}_{s}, \hat{p}_{s}-\nu_{\mathbf{a}} d_{s}}^{\left(d_{s}\right.}
$$

If $\nu_{\mathbf{a}}=0$, the last property in (2.33) imposes no restriction on $d$. In this case, we find that

$$
\sum_{d=0}^{\infty} \mathrm{c}_{\mathbf{p}, \mathbf{b}}^{(d, t)} q^{d}=\left(\begin{array}{c}
N-3 \\
\mathbf{b}
\end{array}\right) \frac{L(q)^{n(1+t)}}{I_{0}(q)^{2}}
$$


In the $\nu_{\mathbf{a}}=0$ case, the last property in (2.33) forces $t \geq 0$ and $t_{\mathbf{p}}=0$ if $t=0$, whenever $|\mathbf{b}|=N-3$. The proof of Theorem $\mathrm{A}$ implies that the right-hand side of (2.39) also vanishes if either $t<0$ or $t=0$ and $t_{\mathbf{p}}>0$. By (2.38) and the last property in (2.33),

$$
(n-l)\left(d^{\prime}+t+1-t_{\mathbf{p}}\right)-(|\mathbf{a}|-l) d^{\prime}+l t-1 \geq 0
$$

whenever the $d^{\prime}$-summand in (2.39) is nonzero; this implies that

$$
1 \leq t_{\mathbf{p}}-t \leq d^{\prime}
$$

whenever the triple product in (2.39) is nonzero and either $t<0$ or $t=0$ and $t_{\mathbf{p}}>0$. The explicit expression on the right-hand side of (2.39) thus provides a direct reason for the vanishing of $\mathbf{c}_{\mathbf{p}, \mathbf{b}}^{(d, t)}$ in these cases.

If $N=3$, the only possibly nonzero coefficients in (2.35) are $\mathbf{c}_{\mathbf{p}, \mathbf{0}}^{(d, 0)}$; these are described by (2.39) and (2.40). If $N=4$, the only possibly nonzero coefficients in (2.35) are $\mathrm{c}_{\mathbf{p}, \mathbf{0}}^{(d, 0)}$ and

$$
\mathrm{c}_{\mathbf{p}, 1000}^{(d, 0)}=\mathrm{c}_{\mathbf{p}, 0100}^{(d, 0)}=\mathrm{c}_{\mathbf{p}, 0010}^{(d, 0)}=\mathrm{c}_{\mathbf{p}, 0001}^{(d, 0)}
$$

with $\mathbf{p} \in \llbracket n \rrbracket^{4}$; the latter set of coefficients is given by (2.39) and (2.40) whenever $\mathbf{p}$ satisfies the last property in (2.33) with $N=4,|\mathbf{b}|=1$, and $t=0$. We next give a formula for the former set of coefficients. For $p, d \in \mathbb{Z}$, define

$$
\begin{gathered}
\llbracket p \rrbracket_{d}, \llbracket \hat{p} \rrbracket_{d}, \tau_{d}(p), t_{d}(p) \in \mathbb{Z} \quad \text { by } \\
0 \leq \llbracket p \rrbracket_{d}, \llbracket \hat{p} \rrbracket_{d} \leq n-1, \quad \llbracket p \rrbracket_{d}+\nu_{\mathbf{a}} d+n \tau_{d}(p)=p, \quad \llbracket p \rrbracket_{d}+\llbracket \hat{p} \rrbracket_{d}+n t_{d}(p)=n-1+l .
\end{gathered}
$$

If $\mathbf{p}, \mathbf{d} \in \mathbb{Z}^{4}$, let

$$
\Sigma_{2}(\mathbf{p}, \mathbf{d})=\left\{p_{1}+p_{2}+\nu_{\mathbf{a}}\left(d_{1}+d_{2}\right), p_{1}+p_{3}+\nu_{\mathbf{a}}\left(d_{1}+d_{3}\right), p_{2}+p_{3}+\nu_{\mathbf{a}}\left(d_{2}+d_{3}\right)\right\} .
$$

If $\nu_{\mathbf{a}}=0, \llbracket p \rrbracket_{d}, \llbracket \hat{p} \rrbracket_{d}$, and $\Sigma_{2}(\mathbf{p}, \mathbf{d})$ do not depend on $d$ or $\mathbf{d}$, and so we omit $d$ and $\mathbf{d}$ from the notation in this case. In the $\nu_{\mathbf{a}}=0$ case, a direct computation from (2.32), (2.40), (2.31), (2.26), and (2.27) gives

$$
\sum_{d=0}^{\infty} \mathrm{c}_{\mathbf{p}, \mathbf{0}}^{(d, 0)} q^{d}=\frac{L(q)^{n+1}}{I_{0}(q)^{2}}\left\{\sum_{p^{\prime}-1 \in \Sigma_{2}(\mathbf{p})} \mathbb{A}_{\llbracket \hat{p}^{\prime} \rrbracket-l}^{(1)}(q)-\sum_{s=1}^{s=4} \mathbb{A}_{\hat{p}_{s}-l}^{(1)}(q)\right\},
$$

whenever $\mathbf{p}$ satisfies the last property in (2.33) with $N=4,|\mathbf{b}|=0$, and $t=0$.

If $\nu_{\mathbf{a}} \neq 0, d, d^{\prime}, p \in \overline{\mathbb{Z}}^{+}$, and $t=0,1$, let

$$
\begin{aligned}
\tilde{\mathrm{c}}_{p, d^{\prime}}^{(d, t)} & \equiv \llbracket \frac{n L(q)^{\nu_{\mathbf{a}} d^{\prime}+n(1-t)}}{|\mathbf{a}|+\nu_{\mathbf{a}} L(q)^{n}}\left(\tilde{\mathrm{c}}_{p, p-\nu_{\mathbf{a}} d}^{(d)} L(q) \mathbb{A}_{p-l-\nu_{\mathbf{a}} d}^{(1)}(q)+\tilde{\mathbf{c}}_{p, p-\nu_{\mathbf{a}} d-1}^{(d)}\right) \|_{q ; d^{\prime}} \\
& =\tilde{\mathrm{c}}_{p, p-\nu_{\mathbf{a}} d}^{(d)} \llbracket \frac{n L(q)^{\nu_{\mathbf{a}} d^{\prime}+n(1-t)+1}}{|\mathbf{a}|+\nu_{\mathbf{a}} L(q)^{n}} \mathbb{A}_{p-l-\nu_{\mathbf{a}} d}^{(1)}(q) \|_{q ; d^{\prime}}+\left(\begin{array}{c}
d^{\prime}-t \\
d^{\prime}
\end{array}\right)\left(\mathbf{a}^{\mathbf{a}}\right)^{d^{\prime}} \tilde{\mathrm{c}}_{p, p-\nu_{\mathbf{a}} d-1}^{(d)} ;
\end{aligned}
$$


the equality above holds by Lemma B.4. On the other hand, by the second equation in (2.28), (2.11), (2.2), and Corollary B.5,

$$
\begin{aligned}
& \llbracket \frac{n L(q)^{\nu_{\mathbf{a}} d^{\prime}+n(1-t)+1}}{|\mathbf{a}|+\nu_{\mathbf{a}} L(q)^{n}} \mathbb{A}_{p-l}^{(1)}(q) \|_{q ; d^{\prime}}
\end{aligned}
$$

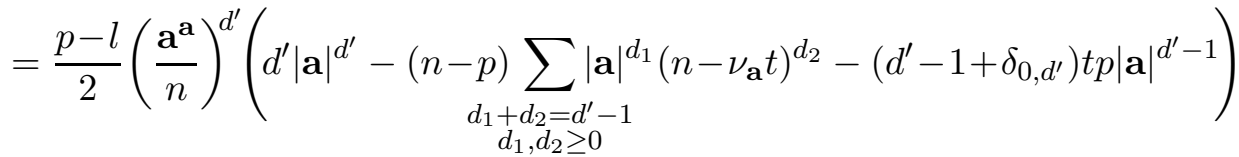

whenever $t=0,1$. In particular, $\tilde{\mathrm{c}}_{p, 0}^{(0, t)}=0$. For $d, p \in \overline{\mathbb{Z}}^{+}$such that $p \leq 2 n-1$, let

$$
\tilde{\mathrm{C}}_{p}^{(d)}=\sum_{\mathbf{d} \in \mathcal{P}_{4}(d)}\left(\mathbf{a}^{\mathbf{a}}\right)^{d_{3}}\left(\begin{array}{c}
d_{3}+\tau_{d_{2}+d_{3}}(p)-t_{d_{2}+d_{3}}(p) \\
d_{3}
\end{array}\right) \tilde{\mathbf{c}}_{\llbracket \hat{p} \rrbracket_{d_{2}+d_{3}}, \llbracket \hat{p} \rrbracket_{d_{2}+d_{3}}-\nu_{\mathbf{a}} d_{2}}^{\left(d_{2}\right)} \tilde{\mathrm{c}}_{\llbracket p \rrbracket_{d_{2}+d_{3}}, d_{4}}^{\left(d_{1}, \tau_{d_{2}+d_{3}}(p)\right)} .
$$

Since $0 \leq p \leq 2 n-1$,

$$
\left(\begin{array}{c}
d_{3}+\tau_{d_{2}+d_{3}}(p)-t_{d_{2}+d_{3}}(p) \\
d_{3}
\end{array}\right) \tilde{\mathrm{c}}_{\llbracket \hat{p} \rrbracket_{d_{2}+d_{3}}, \llbracket\left[\hat{p} \rrbracket_{d_{2}+d_{3}}-\nu_{\mathbf{a}} d_{2}\right.}^{\left(d_{2}\right)} \neq 0 \quad \Longrightarrow \quad \tau_{d_{2}+d_{3}}(p) \in\{0,1\}
$$

by (2.38), and so $\tilde{\mathrm{C}}_{p}^{(d)}$ is well-defined. For example, $\tilde{\mathrm{C}}_{p}^{(0)}=0$. If $\nu_{\mathbf{a}} \neq 0, t_{\mathbf{p}}=0$, and $\mathbf{p}$ satisfies the last property in (2.33) with $N=4,|\mathbf{b}|=0$, and $t=0$, then

$$
\mathrm{c}_{\mathbf{p}, \mathbf{0}}^{(d, 0)}=\sum_{d^{\prime}=0}^{d^{\prime}=d} \sum_{\mathbf{d} \in \mathcal{P}_{4}\left(d-d^{\prime}\right)}\left(\sum_{2 n-2+l-p^{\prime} \in \Sigma_{2}(\mathbf{p}, \mathbf{d})} \tilde{\mathbf{C}}_{\tilde{\mathbf{p}}^{\prime}}^{\left(d^{\prime}\right)} \tilde{\mathrm{c}}_{\hat{\mathbf{p}}}^{(\mathbf{d})}-\sum_{r=1}^{r=4}\left(\prod_{s \in[4]-r} \tilde{\mathrm{c}}_{\hat{p}_{s}, \hat{p}_{s}-\nu_{\mathbf{a}} d_{s}}^{\left(d_{s}\right)}\right) \tilde{\mathrm{c}}_{\hat{p}_{r}, d^{\prime}}^{\left(d_{r}, 0\right)}\right) .
$$

This is obtained by a direct computation from (2.32), (2.39), (2.31), (2.26), and (2.27) except the vanishing of the coefficient of $\Phi_{1}(q)$ follows from Corollary B.8, If $\tilde{\mathrm{c}}_{\hat{\mathbf{p}}}^{(\mathbf{d})} \neq 0$ in (2.43), then

$$
l \leq p_{s}+\nu_{\mathbf{a}} d_{s} \leq n-1 \quad \forall s \in[4]
$$

by the assumption that $t_{\mathbf{p}}=0$ and (2.38), and so

$$
l \leq p^{\prime} \leq 2 n-2-l \quad \text { if } \quad 2 n-2+l-p^{\prime} \in \Sigma_{2}(\mathbf{p}, \mathbf{d}) ;
$$

thus, the right-hand side of $(2.43)$ is well-defined. In the case of a projective space, $\mathbf{a}=\emptyset$, the above formulas give

$$
\begin{gathered}
\tilde{\mathrm{c}}_{p, d^{\prime}}^{(d, t)}=\left\{\begin{array}{ll}
-\frac{p(n-p)}{2 n}, & \text { if } d=0, d^{\prime}>0, t=0 ; \\
-\frac{p(n-p)}{2 n}, & \text { if }\left(d, d^{\prime}, t\right)=(0,1,1) ; \\
0, & \text { otherwise }
\end{array} \tilde{\mathrm{C}}_{p}^{(d)}= \begin{cases}-\frac{\llbracket p \rrbracket_{0}\left(n-\llbracket p \rrbracket_{0}\right)}{2 n}, & \text { if } d>0 ; \\
0, & \text { if } d=0 ;\end{cases} \right. \\
\mathbf{c}_{\mathbf{p}, \mathbf{0}}^{(d, 0)}= \begin{cases}0, & \text { if } d=0,2 ; \\
\min \left\{p_{s}+1, n-1-p_{s}\right\}, & \text { if } d=1 ;\end{cases}
\end{gathered}
$$

the last statement holds under the assumption that $|\mathbf{p}|+n d=3 n-4$.

The $N$-pointed formula of Theorem $₫$ takes the simplest form in the two extremal cases, $\nu_{\mathbf{a}}=0$ (Calabi-Yau) and $\nu_{\mathbf{a}}=n$ (projective space), as $\tilde{\mathrm{c}}_{p, s}^{(d)}=\delta_{0, d} \delta_{p, s}$ in these two cases. However, it is also 
straightforward to compute all the relevant coefficients in the intermediate cases. For example, for a cubic threefold $X_{3} \subset \mathbb{P}^{4}$, the only non-trivial coefficients $\tilde{\mathrm{c}}_{p, s}^{(d)}$ are

$$
\tilde{\mathrm{c}}_{3,1}^{(1)}=\tilde{\mathrm{c}}_{4,1}^{(1)}=-6, \quad \tilde{\mathrm{c}}_{4,2}^{(1)}=-21,
$$

as computed in [24, Section 2] 9 From this, (2.39), and (2.43), we find that the only nonzero coefficients in the $N=3,4$ cases of (2.35) with $d \in \mathbb{Z}^{+}$and $\mathbf{b}=\mathbf{0}$ are

$$
\begin{array}{lllll}
c_{133,0}^{(1,0)}=6, & c_{223, \mathbf{0}}^{(1,0)}=15, & c_{113, \mathbf{0}}^{(2,0)}=36, & c_{122, \mathbf{0}}^{(2,0)}=126, & c_{111, \mathbf{0}}^{(3,0)}=216, \\
c_{1333, \mathbf{0}}^{(1,0)}=6, & c_{2233, \mathbf{0}}^{(1,0)}=15, & c_{1133, \mathbf{0}}^{(2,0)}=72, & c_{1223, \mathbf{0}}^{(2,0)}=252, & c_{1113, \mathbf{0}}^{(3,0)}=648, \\
& & c_{2222, \mathbf{0}}^{(2,0)}=729, & c_{1122, \mathbf{0}}^{(3,0)}=2484, & c_{111, \mathbf{0}}^{(4,0)}=5184,
\end{array}
$$

up to the permutations of the first three subscripts. From (2.35), we then find that

$$
\begin{array}{rrr}
\left\langle H^{3}, H, H\right\rangle_{0,1}^{X_{3}}=\left\langle H^{3}, H, H, H\right\rangle_{0,1}^{X_{3}}=18, & \left\langle H^{2}, H^{2}, H\right\rangle_{0,1}^{X_{3}}=\left\langle H^{2}, H^{2}, H, H\right\rangle_{0,1}^{X_{3}}=45, \\
\left\langle H^{3}, H^{3}, H\right\rangle_{0,2}^{X_{3}}=\frac{1}{2}\left\langle H^{3}, H^{3}, H, H\right\rangle_{0,2}^{X_{3}}=108, & \left\langle H^{3}, H^{2}, H^{2}\right\rangle_{0,2}^{X_{3}}=\frac{1}{2}\left\langle H^{3}, H^{2}, H^{2}, H\right\rangle_{0,2}^{X_{3}}=378, \\
\left\langle H^{2}, H^{2}, H^{2}, H^{2}\right\rangle_{0,2}^{X_{3}}=2187, & \left\langle H^{3}, H^{3}, H^{3}\right\rangle_{0,3}^{X_{3}}=\frac{1}{3}\left\langle H^{3}, H^{3}, H^{3}, H\right\rangle_{0,3}^{X_{3}}=648, \\
\left\langle H^{3}, H^{3}, H^{2}, H^{2}\right\rangle_{0,3}^{X_{3}}=7452, & \left\langle H^{3}, H^{3}, H^{3}, H^{3}\right\rangle_{0,4}^{X_{3}}=15552 .
\end{array}
$$

These conclusions are consistent with the divisor relation. The above invariants are enumerative at least for $d=1,2,3$. The degree 1 and 2 numbers agree with the classical Schubert calculus computations on $G(2,5)$ and $G(3,5)$, respectively. The approach of $[8$ can be used to test the two degree 3 numbers.

Based on (2.32), the coefficient $\mathrm{c}_{\mathbf{p}, \mathbf{b}}^{(d, 0)}$ in (2.35) with $\mathbf{p} \in \llbracket n \rrbracket^{N}$ involves the power series $\Phi_{r}$ of Proposition 2.1 with $r=0,1, \ldots, N-3-|\mathbf{b}|$ only. By (2.42) and (2.43), only the power series $\Phi_{0}$ enters in the $N=4$ case. For $N=5$, the power series $\Phi_{1}$ and $\Phi_{2}$ do enter in the final expression for $\mathrm{c}_{\mathbf{p}, \mathbf{0}}^{(d, 0)}$. However, at least for $\mathbf{a}=(n)$, i.e. when $X_{\mathbf{a}}$ is a Calabi-Yau hypersurface, $\Phi_{2}$ cancels with $\Phi_{1}^{2} / \Phi_{0}$ (these two power series are equal in this case).

\subsection{Alternative description of the structure constants}

We now describe the constants $\mathbf{c}_{\mathbf{p}, \mathbf{b}}^{(d, 0)}$ defined above as sums over $N$-marked trivalent trees 10 It is fairly straightforward to see that the two descriptions are equivalent; this also follows from the two variations of the main localization computation in Section 4 .

A graph consists of a set Ver of vertices and a collection Edg of edges, i.e. of two-element subsets of Ver. In Figure 1, the vertices are represented by dots, while each edge $\left\{v_{1}, v_{2}\right\}$ is shown as the line segment between $v_{1}$ and $v_{2}$. For such a graph $\Gamma$ and $v \in$ Ver, let

$$
\mathrm{E}_{v}(\Gamma)=\{e \in \operatorname{Edg}: v \in e\}
$$

\footnotetext{
${ }^{9}$ In this paper, the subscripts on $\tilde{c}$ are shifted up by $l$ from 24 .

${ }^{10}$ The constants $\mathrm{c}_{\mathbf{p}, \mathbf{b}}^{(d, t)}$ with $t>0$ can be described in the same way as well, but are not needed in this approach.
} 

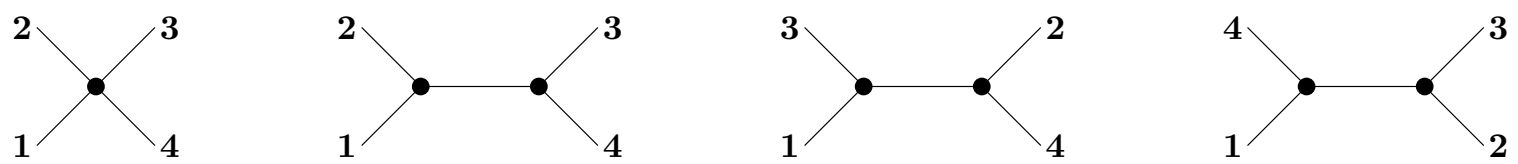

Figure 1: The trivalent 4-marked trees

be the set of edges leaving $v$. A graph (Ver, Edg) is a tree if it contains no loops, i.e. the set Edg contains no $m$-element subset of the form

$$
\left\{\left\{v_{1}, v_{2}\right\},\left\{v_{2}, v_{3}\right\}, \ldots,\left\{v_{m}, v_{1}\right\}\right\}, \quad v_{1}, \ldots, v_{m} \in \text { Ver, } m \geq 3
$$

all four graphs in Figure 1 are trees. An $N$-marked graph is a tuple $\Gamma=($ Ver, Edg; $\eta$ ), where (Ver, Edg) is a graph and $\eta:[N] \longrightarrow$ Ver is a map; in Figure 1, the elements of the set $[N]=[4]$ are shown in bold face and are linked by line segments to their images under $\eta$. An $n$-marked graph $\Gamma=($ Ver, Edg; $\eta)$ is called trivalent if

$$
m_{v} \equiv \operatorname{val}_{\Gamma}(v)-3 \equiv\left|\mathrm{E}_{\Gamma}(v)\right|+\left|\eta^{-1}(v)\right|-3 \geq 0
$$

for every vertex $v \in$ Ver. There is a unique trivalent 3-marked tree; the four trivalent 4-marked trees are shown in Figure 1. For any $N$-marked tree,

$$
\sum_{v \in \text { Ver }} m_{v}+|\mathrm{Edg}|=N-3
$$

We will call a partial ordering $\prec$ on a set Ver linear if for any pair of distinct incomparable elements $v_{1}, v_{2} \in$ Ver there exists a third element $v \in$ Ver such that $v \prec v_{1}, v_{2}$. A finite linearly ordered set Ver has a unique minimal element $v_{0} \in$ Ver. For each trivalent $N$-marked tree $\Gamma=(\operatorname{Ver}, \mathrm{Edg} ; \eta)$, we fix a partial ordering $\prec$ on Ver so that if $v \prec v^{\prime}$, then there exist

$$
v_{1}, \ldots, v_{m} \in \operatorname{Ver} \quad \text { s.t. } \quad v_{i-1} \prec v_{i}, \quad\left\{v_{i-1}, v_{i}\right\} \in \operatorname{Edg} \forall i \in[m+1], \quad \text { where } v_{0} \equiv v, v_{m+1} \equiv v^{\prime} .11
$$

For every edge $e \in \operatorname{Edg}$, let $v_{e}^{-}, v_{e}^{+} \in \operatorname{Ver}$ be the elements of $e \subset$ Ver with $v_{e}^{-} \prec v_{e}^{+}$. For each $v \in \operatorname{Ver}$, let

$$
\mathrm{E}_{\Gamma}^{-}(v)=\left\{e \in \operatorname{Edg}: v_{e}^{-}=v\right\}
$$

be the set of edges descending to $v$. If $v \neq v_{0}$, let $e_{v} \in \operatorname{Edg}$ be the unique edge descending from $v$.

Let $(\mathbf{p}, \mathbf{b}, d) \in \llbracket n \rrbracket_{l}^{N} \times\left(\overline{\mathbb{Z}}^{+}\right)^{N} \times \overline{\mathbb{Z}}^{+}$be a tuple satisfying the two properties on the right-hand side of (2.33) with $t=0, \Gamma=($ Ver, Edg; $\eta)$ be a trivalent $N$-marked tree, and

$$
\mathbf{d} \equiv\left(d_{v}\right)_{v \in \text { Ver }} \in \mathcal{P}_{\Gamma}(d) \equiv \mathcal{P}_{\text {Ver }}(d)
$$

be a partition of $d$ into nonnegative integers. We denote by

$$
\mathcal{S}_{\Gamma}(\mathbf{p}, \mathbf{b}, \mathbf{d}) \subset \llbracket n \rrbracket^{\mathrm{Edg}} \times\left(\overline{\mathbb{Z}}^{+}\right)^{\mathrm{Edg}} \times \mathbb{Z}^{\mathrm{Ver}}
$$

\footnotetext{
${ }^{11}$ Such a partial ordering is determined by the minimal vertex $v_{0}$, which could be taken to be $\eta(N)$, for example.
} 
the subset of triples $\left(\mathbf{p}^{\prime}, \mathbf{b}^{\prime}, \mathbf{t}\right)$ such that

$$
\sum_{s \in \eta^{-1}(v)}\left(\hat{p}_{s}+b_{s}\right)+\sum_{e \in \mathrm{E}_{\Gamma}^{-}(v)}\left(\hat{p}_{e}^{\prime}-1-b_{e}^{\prime}\right)+\left(p_{e_{v}}^{\prime}+b_{e_{v}}^{\prime}\right)=n-3+\left(m_{v}+2\right)(l+1)+\nu_{\mathbf{a}} d_{v}+n t_{v}
$$

for all $v \in$ Ver, where $\hat{p}$ is as in (2.36) and we set $p_{e_{v}}^{\prime}+b_{e_{v}} \equiv 0$ if $v=v_{0}$. Each choice of $\mathbf{b}^{\prime}$ determines $\mathbf{p}^{\prime}$ and $\mathbf{t}$ uniquely by solving (2.46) for $p_{v}$ and $t_{v}$ starting with maximal elements of Ver and moving down; the equation for $v=v_{0}$ will then be automatically solvable for $t_{v}$ because of the last property in (2.33). Furthermore, for every $\left(\mathbf{p}^{\prime}, \mathbf{b}^{\prime}, \mathbf{t}\right) \in \mathcal{S}_{\Gamma}(\mathbf{p}, \mathbf{b}, \mathbf{d})$

$$
t_{\mathbf{p}^{\prime}}+\sum_{v \in \text { Ver }} t_{v}=0
$$

with $t_{\mathbf{p}^{\prime}}$ as in (2.37).

If $(\mathbf{p}, \mathbf{b}) \in \llbracket n \rrbracket_{l}^{N} \times\left(\overline{\mathbb{Z}}^{+}\right)^{N}$ and $d \in \overline{\mathbb{Z}}^{+}$satisfy the last property in (2.33) with $t=0$, set

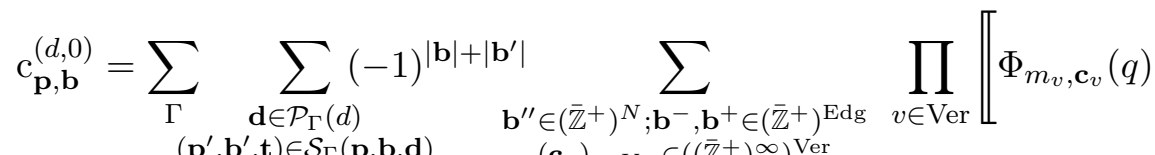

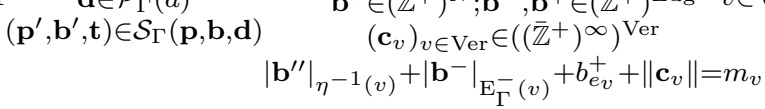

$$
\begin{aligned}
& \times \prod_{s \in \eta^{-1}(v)} \frac{\Phi_{\hat{p}_{s} ; b_{s}^{\prime \prime}-b_{s}}(q)}{b_{s}^{\prime \prime} ! \Phi_{0}(q)} \times \prod_{e \in \mathrm{E}_{\Gamma}^{-}(v)} \frac{L(q)^{\delta_{0 \nu_{\mathbf{a}}} n t_{p_{e}^{\prime}} \Phi_{\hat{p}_{e}^{\prime} ; b_{e}^{-}+1+b_{e}^{\prime}}(q)}}{b_{e}^{-} ! \Phi_{0}(q)} \times \frac{I_{0}(q)^{2} \Phi_{p_{e_{v}}^{\prime} ; b_{e v}^{+}-b_{e v}^{\prime}}(q)}{b_{e_{v}}^{+} ! L(q)^{\delta_{0 \nu_{\mathbf{a}}} n} \Phi_{0}(q)} \|_{q ; d_{v}}
\end{aligned}
$$

where $b_{e_{v_{0}}}^{+} \equiv 0$, the last fraction is defined to be 1 for $v=v_{0}$, and the outer sum is taken over all trivalent $N$-marked trees $\Gamma=(\mathrm{Ver}, \mathrm{Edg} ; \eta)$. For example, the contribution of the one-vertex $N$-marked tree is

$$
(-1)^{|\mathbf{b}|} \sum_{\substack{\mathbf{b}^{\prime \prime} \in\left(\overline{\mathbb{Z}}^{+}\right)^{N}, \mathbf{c} \in\left(\overline{\mathbb{Z}}^{+}\right)^{\infty} \\\left|\mathbf{b}^{\prime \prime}\right|+\|\mathbf{c}\|=N-3}} \llbracket \Phi_{N-3, \mathbf{c}}(q) \prod_{s=1}^{s=N} \frac{\Phi_{\hat{p}_{s} ; b_{s}^{\prime \prime}-b_{s}}(q)}{b_{s}^{\prime \prime} ! \Phi_{0}(q)} \|_{q ; d}
$$

If $|\mathbf{b}|=N-3$, this gives (2.39) and (2.40) with $t, t_{\mathbf{p}}=0$.

For a nonzero summand in (2.47),

$$
b_{s} \leq b_{s}^{\prime \prime} \forall s \in[N] \quad \text { and } \quad\left|\mathbf{b}^{\prime \prime}\right| \leq N-3-|\mathrm{Edg}|
$$

the latter inequality follows from (2.45). This implies the bound on $\mathbf{b}$ in (2.33). If $d \in \overline{\mathbb{Z}}^{+}$and $(\mathbf{p}, \mathbf{b}) \in \llbracket n \|_{l}^{N} \times\left(\overline{\mathbb{Z}}^{+}\right)^{N}$ do not satisfy the last condition in $(2.33)$ with $t=0$, set $\mathbf{c}_{\mathbf{p}, \mathbf{b}}^{(d, 0)}=0$.

In the Calabi-Yau case, $\nu_{\mathbf{a}}=0$, the collection $\mathcal{S}_{\Gamma}(\mathbf{p}, \mathbf{b}) \equiv \mathcal{S}_{\Gamma}(\mathbf{p}, \mathbf{b}, \mathbf{d})$ does not depend on $\mathbf{d}$. In the projective case, $\nu_{\mathbf{a}}=n$, the collection of pairs $\left(\mathbf{p}^{\prime}, \mathbf{b}^{\prime}\right)$ does not depend on $\mathbf{d}$. As $t_{v}$ in (2.46) is determined by $\mathbf{b}^{\prime}$, we abbreviate the elements of $\mathcal{S}_{\Gamma}(\mathbf{p}, \mathbf{b})$ as $\left(\mathbf{p}^{\prime}, \mathbf{b}^{\prime}\right)$ in either case. In these 
extremal cases, (2.30) and (2.12) reduce (2.47) to

$$
\begin{aligned}
& \mathrm{c}_{\mathbf{p}, \mathbf{b}}^{(d, 0)}=\sum_{\Gamma} \sum_{\left(\mathbf{p}^{\prime}, \mathbf{b}^{\prime}\right) \in \mathcal{S}_{\Gamma}(\mathbf{p}, \mathbf{b})}(-1)^{|\mathbf{b}|+\left|\mathbf{b}^{\prime}\right|} \sum_{\mathbf{b}^{\prime \prime} \in\left(\overline{\mathbb{Z}}^{+}\right)^{N} ; \mathbf{b}^{-}, \mathbf{b}^{+} \in\left(\overline{\mathbb{Z}}^{+}\right)^{\mathrm{Edg}}} \llbracket L(q)^{|\mathbf{a}| t_{\mathbf{p}^{\prime}}} \Phi_{\Gamma,\left(\mathbf{c}_{v}\right)_{v \in \mathrm{Ver}}}(q) \\
& \left(\mathbf{c}_{v}\right)_{v \in \operatorname{Ver}} \in\left(\left(\overline{\mathbb{Z}}^{+}\right)^{\infty}\right) \text { Ver } \\
& \left|\mathbf{b}^{\prime \prime}\right|_{\eta^{-1}(v)}+\left|\mathbf{b}^{-}\right|_{\mathbf{e}_{\Gamma}^{-}(v)}+b_{e_{v}}^{+}+\left\|\mathbf{c}_{v}\right\|=m_{v} \\
& \times \prod_{s=1}^{s=N} \frac{\Phi_{\hat{p}_{s} ; b_{s}^{\prime \prime}-b_{s}}(q)}{b_{s}^{\prime \prime} ! \Phi_{0}(q)} \times \prod_{e \in \operatorname{Edg}} \frac{\Phi_{\hat{p}_{e}^{\prime} ; b_{e}^{-}+1+b_{e}^{\prime}}(q) \Phi_{p_{e}^{\prime} ; b_{e}^{+}-b_{e}^{\prime}}(q)}{b_{e}^{-} ! b_{e}^{+} ! \Phi_{0}(q)^{2}} \|_{q ; d},
\end{aligned}
$$

where

$$
\Phi_{\Gamma,\left(\mathbf{c}_{v}\right)_{v \in \mathrm{Ver}}}=\frac{L^{|\mathbf{a}|-(n-1-l)|\mathrm{Ver}|}}{I_{0}^{2}} \prod_{v \in \mathrm{Ver}}\left((-1)^{m_{v}+\left|\mathbf{c}_{v}\right|}\left(m_{v}+\left|\mathbf{c}_{v}\right|\right) ! \prod_{r=1}^{\infty} \frac{1}{c_{v ; r} !}\left(\frac{\Phi_{r}}{(r+1) ! \Phi_{0}}\right)^{c_{v ; r}}\right) .
$$

The coefficients $\mathbf{c}_{\mathbf{p}, \mathbf{b}}^{(d, 0)}$ must be invariant under the permutations of $[N]$ (same permutations in the components of $\mathbf{p}$ and $\mathbf{b}$ ). For $N \geq 4$, this is not apparent from either of the above two descriptions of these coefficients, even in the extremal cases; thus, this is a consequence of the proof of Theorem $\mathrm{A}$ below. In the case of (1.8), this invariance can be seen directly using Lemma 2.3, as indicated in Section 1.1 .

\section{Equivariant GW-invariants}

In this section we first review the relevant aspects of equivariant cohomology; a more detailed discussion can be found in [27, Section 1.1]. We then state an equivariant version of Theorem $\mathrm{A}$ and use it to obtain Theorem $\mathrm{A}$.

We denote by $\mathbb{T}$ the $n$-torus $\left(\mathbb{C}^{*}\right)^{n}$. Its group cohomology is the polynomial algebra on $n$ generators:

$$
H_{\mathbb{T}}^{*} \equiv H^{*}(B \mathbb{T} ; \mathbb{Q})=\mathbb{Q}[\alpha] \equiv \mathbb{Q}\left[\alpha_{1}, \ldots, \alpha_{n}\right],
$$

where $\alpha=\left(\alpha_{1}, \ldots, \alpha_{n}\right)$ and $\alpha_{i}=\pi_{i}^{*} c_{1}\left(\gamma^{*}\right)$ if

$$
\pi_{i}: B \mathbb{T} \longrightarrow B \mathbb{C}^{*}=\mathbb{P}^{\infty} \quad \text { and } \quad \gamma \longrightarrow \mathbb{P}^{\infty}
$$

are the projection onto the $i$-th component and the tautological line bundle, respectively. Let

$$
\mathcal{H}_{\mathbb{T}}^{*}=\mathbb{Q}_{\alpha} \equiv \mathbb{Q}\left(\alpha_{1}, \ldots, \alpha_{n}\right) \quad \text { and } \quad \mathcal{I} \subset \mathbb{Q}\left[\alpha_{1}, \ldots, \alpha_{n}\right] \subset \mathcal{H}_{\mathbb{T}}^{*}
$$

be the field of fractions of $H_{\mathbb{T}}^{*}$ and the ideal in $\mathbb{Q}[\underline{\alpha}]$ generated by the elementary symmetric polynomials $\sigma_{1}, \sigma_{2}, \ldots, \sigma_{n-1}$ in $\alpha_{1}, \alpha_{2}, \ldots, \alpha_{n}$, respectively. Let

$$
\hat{\sigma}_{r}=(-1)^{r-1} \sigma_{r} \in \mathbb{Q}_{\alpha} \quad r=0,1,2, \ldots, \quad D_{\alpha}=\prod_{j \neq k}\left(\alpha_{j}-\alpha_{k}\right) .
$$


If $\mathbb{T}$ is acting on a topological space $M$, let

$$
H_{\mathbb{T}}^{*}(M) \equiv H^{*}(B M ; \mathbb{Q}), \quad \text { where } \quad B M=E \mathbb{T} \times_{\mathbb{T}} M,
$$

be the equivariant cohomology of $M$. The projection map $B M \longrightarrow B \mathbb{T}$ induces an action of $H_{\mathbb{T}}^{*}$ on $H_{\mathbb{T}}^{*}(M)$. We define

$$
\mathcal{H}_{\mathbb{T}}^{*}(M)=H_{\mathbb{T}}^{*}(M) \otimes_{H_{\mathbb{T}}^{*}} \mathcal{H}_{\mathbb{T}}^{*} .
$$

If the $\mathbb{T}$-action on $M$ lifts to an action on a (complex) vector bundle $V \longrightarrow M$, let

$$
\mathbf{e}(V) \equiv e(B V) \in H_{\mathbb{T}}^{*}(M) \subset \mathcal{H}_{\mathbb{T}}^{*}(M)
$$

denote the equivariant euler class of $V$.

Throughout the paper we work with the standard action of $\mathbb{T}$ on $\mathbb{P}^{n-1}$ :

$$
\left(e^{\mathrm{i} \theta_{1}}, \ldots, e^{\mathrm{i} \theta_{n}}\right) \cdot\left[z_{1}, \ldots, z_{n}\right]=\left[e^{\mathrm{i} \theta_{1}} z_{1}, \ldots, e^{\mathrm{i} \theta_{n}} z_{n}\right]
$$

it has $n$ fixed points:

$$
P_{1}=[1,0, \ldots, 0], \quad P_{2}=[0,1,0, \ldots, 0], \quad \ldots \quad P_{n}=[0, \ldots, 0,1] .
$$

The $\mathbb{T}$-equivariant cohomology of $\mathbb{P}_{N}^{n-1}$ with respect to the induced diagonal $\mathbb{T}$-action on $\mathbb{P}_{N}^{n-1}$ is given by

$$
H_{\mathbb{T}}^{*}\left(\mathbb{P}_{N}^{n-1}\right)=\mathbb{Q}[\alpha, \underline{\mathbf{x}}] /\left\{\left(\mathbf{x}_{s}-\alpha_{1}\right) \ldots\left(\mathbf{x}_{s}-\alpha_{n}\right): s=1, \ldots, N\right\}
$$

where $\underline{\mathbf{x}}=\left(\mathbf{x}_{1}, \ldots, \mathbf{x}_{n}\right)$ and $\mathbf{x}_{s}=\pi_{s}^{*} \mathbf{x}$ if $\pi_{s}: \mathbb{P}_{N}^{n-1} \longrightarrow \mathbb{P}^{n-1}$ is the projection onto the $s$-th component and $\mathbf{x} \in H_{\mathbb{T}}^{*}\left(\mathbb{P}^{n-1}\right)$ is the equivariant hyperplane class. For each $\mathbf{p} \in\left\lfloor n \rrbracket^{N}\right.$, let

$$
\underline{\mathbf{x}}^{\mathbf{p}}=\prod_{i=1}^{i=N} \mathbf{x}_{s}^{p_{s}} \in H_{\mathbb{T}}^{*}\left(\mathbb{P}_{N}^{n-1}\right)
$$

these elements form a basis for $H_{\mathbb{T}}^{*}\left(\mathbb{P}_{N}^{n-1}\right)$ as a module over $H_{\mathbb{T}}^{*}=\mathbb{Q}[\alpha]$.

The action of $\mathbb{T}$ on $\mathbb{P}^{n-1}$ naturally lifts to the tautological line bundle $\gamma$, the vector bundle

$$
\mathcal{L} \equiv \bigoplus_{k=1}^{k=l} \gamma^{* \otimes a_{k}}=\bigoplus_{k=1}^{k=l} \mathcal{O}_{\mathbb{P}^{n-1}}\left(a_{k}\right) \longrightarrow \mathbb{P}^{n-1}
$$

and the tangent bundle $T \mathbb{P}^{n-1}$ so that

$$
\left.\mathbf{e}(\mathcal{L})\right|_{P_{i}}=\langle\mathbf{a}\rangle \alpha_{i}^{l},\left.\quad \mathbf{e}\left(T \mathbb{P}^{n-1}\right)\right|_{P_{i}}=\prod_{\substack{1 \leq k \leq n \\ k \neq i}}\left(\alpha_{i}-\alpha_{k}\right) \quad \forall i=1,2, \ldots, n .
$$

Via composition of maps, the action of $\mathbb{T}$ on $\mathbb{P}^{n-1}$ and $\mathcal{L}$ induces actions on $\overline{\mathfrak{M}}_{0, N}\left(\mathbb{P}^{n-1}, d\right)$ and

$$
\mathcal{V}_{d}=\overline{\mathfrak{M}}_{0, N}(\mathcal{L}, d) \longrightarrow \overline{\mathfrak{M}}_{0, N}\left(\mathbb{P}^{n-1}, d\right)
$$

so that the evaluation maps

$$
\mathrm{ev} \equiv \mathrm{ev}_{1} \times \ldots \times \mathrm{ev}_{N}: \overline{\mathfrak{M}}_{0, N}\left(\mathbb{P}^{n-1}, d\right) \longrightarrow \mathbb{P}_{N}^{n-1}, \quad \widetilde{\mathrm{ev}}_{s}: \mathcal{V}_{d} \longrightarrow \mathrm{ev}_{s}^{*} \mathcal{L}, \quad \widetilde{\mathrm{ev}}_{s}([\mathcal{C}, f ; \tilde{f}])=\left[\tilde{f}\left(x_{s}(\mathcal{C})\right)\right]
$$


where $x_{s}(\mathcal{C})$ is the $s$-th marked point of the curve $\mathcal{C}$, are $\mathbb{T}$-equivariant. In particular, $\mathcal{V}_{d}$ has a well-defined equivariant euler class

$$
\mathbf{e}\left(\mathcal{V}_{d}\right) \in H_{\mathbb{T}}^{*}\left(\overline{\mathfrak{M}}_{0, N}\left(\mathbb{P}^{n-1}, d\right)\right)
$$

Since the bundle homomorphisms $\widetilde{\mathrm{ev}}_{s}$ are surjective, their kernels are again equivariant vector bundles. Let

$$
\mathcal{V}_{d}^{\prime \prime}=\operatorname{ker} \widetilde{\mathrm{ev}}_{2} \longrightarrow \overline{\mathfrak{M}}_{0,2}\left(\mathbb{P}^{n-1}, d\right)
$$

With $\underline{\hbar}$ and $\underline{\hbar}^{-1}$ as in (2.1) and $\underline{\mathbf{x}}$ as in (3.1), let

$$
\mathcal{Z}(\underline{\hbar}, \underline{\mathbf{x}}, Q)=\sum_{d=0}^{\infty} Q^{d} \mathrm{ev}_{*}\left\{\frac{\mathbf{e}\left(\mathcal{V}_{d}\right)}{\prod_{s=1}^{s=N}\left(\hbar_{s}-\psi_{s}\right)}\right\} \in H_{\mathbb{T}}^{*}\left(\mathbb{P}_{N}^{n-1}\right)\left[\left[\underline{\hbar}^{-1}, Q\right]\right],
$$

where ev: $\overline{\mathfrak{M}}_{0, N}\left(\mathbb{P}^{n-1}, d\right) \longrightarrow \mathbb{P}_{N}^{n-1}$; for $N=1,2$, we define the coefficient of $Q^{0}$ to be

$$
\langle\mathbf{a}\rangle \mathbf{x}_{1}^{l} \quad \text { and } \quad-\frac{\langle\mathbf{a}\rangle \mathbf{x}_{1}^{l}}{\hbar_{1}+\hbar_{2}} \sum_{\substack{p_{1}+p_{2}+r=n-1 \\ p_{1}, p_{2}, r \geq 0}} \hat{\sigma}_{r} \mathbf{x}_{1}^{p_{1}} \mathbf{x}_{2}^{p_{2}},
$$

respectively. For each $p \in \llbracket n \rrbracket$, let

$$
\mathcal{Z}_{p}(\hbar, \mathbf{x}, Q)=\mathbf{x}^{p}+\sum_{d=1}^{\infty} Q^{d} \operatorname{ev}_{1 *}\left\{\frac{\mathbf{e}\left(\mathcal{V}_{d}^{\prime \prime}\right) \mathrm{ev}_{2}^{*} \mathbf{x}^{p}}{\hbar-\psi}\right\} \in H_{\mathbb{T}}^{*}\left(\mathbb{P}^{n-1}\right)\left[\left[\hbar^{-1}, Q\right]\right]
$$

where $\mathrm{ev}_{1}, \mathrm{ev}_{2}: \overline{\mathfrak{M}}_{0,2}\left(\mathbb{P}^{n-1}, d\right) \longrightarrow \mathbb{P}^{n-1}$. Similarly to (2.20), let

$$
\mathcal{Z}_{\mathbf{p}}(\underline{\hbar}, \underline{\mathbf{x}}, Q) \equiv \prod_{s=1}^{s=N} \frac{1}{\hbar_{s}} \frac{\mathcal{Z}_{p_{s}}\left(\hbar, \mathbf{x}_{s}, Q\right)}{\prod_{r=p_{s}-l+1}^{n-l-1} I_{r}\left(q_{s}\right)}
$$

Theorem B. Suppose $n, N \in \mathbb{Z}^{+}$, with $N \geq 3$, and $\mathbf{a} \in\left(\mathbb{Z}^{+}\right)^{l}$ is such that $\|\mathbf{a}\| \leq n$. The generating function (3.3) for equivariant $N$-pointed genus $0 G W$-invariants of a complete intersection $X_{\mathbf{a}} \subset$ $\mathbb{P}^{n-1}$ is given by

$$
\mathcal{Z}(\underline{\hbar}, \underline{\mathbf{x}}, Q)=\langle\mathbf{a}\rangle \sum_{\mathbf{p} \in \llbracket n \rrbracket^{N}} \sum_{\mathbf{b} \in\left(\overline{\mathbb{Z}}^{+}\right)^{N}} \sum_{d=0}^{\infty} \mathcal{C}_{\mathbf{p}, \mathbf{b}}^{(d)} q^{d} \underline{\hbar}^{-\mathbf{b}} \mathcal{Z}_{\mathbf{p}}(\underline{\hbar}, \underline{\mathbf{x}}, Q)
$$

for some $\mathcal{C}_{\mathbf{p}, \mathbf{b}}^{(d)} \in \mathbb{Q}[\alpha]$ such that

$$
\mathcal{C}_{\mathbf{p}, \mathbf{b}}^{(d)}-\sum_{t=0}^{\infty} \mathrm{c}_{\mathbf{p}, \mathbf{b}}^{(d, t)} \hat{\sigma}_{n}^{t} \in \mathcal{I}
$$

where $\mathbf{c}_{\mathbf{p}, \mathbf{b}}^{(d, t)} \in \mathbb{Q}$ are the numbers defined above Theorem $\underline{A}$. 
Setting $\alpha=0$ in Theorem $B$ and using [24, Theorem 3], we obtain

$$
Z(\underline{\hbar}, \underline{H}, Q)=\langle\mathbf{a}\rangle e^{-\sum_{s=1}^{s=N} J\left(q_{s}\right) w_{s}} \sum_{\mathbf{p} \in\left\lfloor n \rrbracket^{N}\right.} \sum_{\mathbf{b} \in\left(\overline{\mathbb{Z}}^{+}\right)^{N}} \sum_{d=0}^{\infty} c_{\mathbf{p}, \mathbf{b}}^{(d, 0)} q^{d} \underline{\hbar}^{-\mathbf{b}} \Delta_{\mathbf{p}}(\underline{\hbar}, \underline{H}, Q) .
$$

This implies Theorem $\mathrm{A}$ provided $\mathrm{c}_{\mathbf{p}, \mathbf{b}}^{(d, 0)}=0$ if $p_{s}<l$ for some $s \in[N]$; this is shown in the next paragraph.

Suppose instead $\mathbf{c}_{\mathbf{p}, \mathbf{b}}^{(d, 0)}=0$ for some triple $(\mathbf{p}, \mathbf{b}, d)$ with $p_{1}<l$. Choose $(\mathbf{p}, \mathbf{b}, d)$ minimizing $p_{1}$, as well as minimizing $d$ for the smallest possible $p_{1}$. We show that

$$
\left\langle\tau_{b_{1}} H^{n-1-p_{1}}, \ldots, \tau_{b_{N}} H^{n-1-p_{N}}\right\rangle_{0, d}^{X_{\mathbf{a}}}=\langle\mathbf{a}\rangle \mathbf{c}_{\mathbf{p}, \mathbf{b}}^{(d, 0)} .
$$

By (1.3) and (2.1), this GW-invariant is the coefficient of $Q^{d} \prod_{s=1}^{s=N} \hbar_{s}^{-\left(b_{s}+1\right)} H_{s}^{p_{s}}$ of the right-hand side of (3.7). Suppose a triple $\left(\mathbf{p}^{\prime}, \mathbf{b}^{\prime}, d^{\prime}\right)$, with $\mathrm{c}_{\mathbf{p}^{\prime}, \mathbf{b}^{\prime}}^{\left(d^{\prime}, 0\right)} \neq 0$, contributes to this coefficient. Since the lowest power of $H$ in the coefficient of a product of powers of $q$ and $\hbar^{-1}$ in $H^{p} F_{p}(w, q)$ is $\min (p, l)$, $p_{1}^{\prime}=p_{1}$ by the minimality of $p_{1}$ and thus $d^{\prime}=d$ by the minimality of $d$. Since the coefficient of $q^{0}$ in $H^{p} F_{p}(w, q)$ is $H^{p}, p_{s}^{\prime}=p_{s}$ for all $s \in[N]$ and thus $b_{s}^{\prime}=b_{s}$ for all $s \in[N]$; this gives (3.8). Since $\left.H^{n-1-p_{1}}\right|_{X_{\mathbf{a}}}=0$ for $p_{1}<l$, we conclude that $\mathrm{c}_{\mathbf{p}, \mathbf{b}}^{(d, 0)}=0$.

The proof of Theorem $\mathrm{B}$ below provides an algorithm for computing the structure coefficients $\mathcal{C}_{\mathbf{p}, \mathbf{b}}^{(d)}$ completely. On the other hand, they may be irrelevant in many applications. For example, the oneand two-point equivariant generating functions (3.3) play a key in the localization computation of the genus $1 \mathrm{GW}$-invariants of Calabi-Yau complete intersections in [27] and in [23], but the structure coefficients lying in $\mathcal{I}$ are ignored. Similarly, the equivariant generating functions with $N \leq g$ and the structure coefficients lying in $\mathcal{I}$ dropped should play a key role in computing genus $g \geq 2$ GW-invariants of complete intersections.

\section{Proof of Theorem $\mathrm{A}$}

\subsection{Localization Setup}

If $\mathbb{T}$ acts smoothly on a smooth compact oriented manifold $M$, there is a well-defined integrationalong-the-fiber homomorphism

$$
\int_{M}: H_{\mathbb{T}}^{*}(M) \longrightarrow H_{\mathbb{T}}^{*}
$$

for the fiber bundle $B M \longrightarrow B \mathbb{T}$. The classical localization theorem of [3] relates it to integration along the fixed locus of the $\mathbb{T}$-action. The latter is a union of smooth compact orientable manifolds $F$ and $\mathbb{T}$ acts on the normal bundle $\mathcal{N} F$ of each $F$. Once an orientation of $F$ is chosen, there is a well-defined integration-along-the-fiber homomorphism

$$
\int_{F}: H_{\mathbb{T}}^{*}(F) \longrightarrow H_{\mathbb{T}}^{*}
$$

The localization theorem states that

$$
\int_{M} \psi=\sum_{F} \int_{F} \frac{\left.\psi\right|_{F}}{\mathbf{e}(\mathcal{N} F)} \in \mathcal{H}_{\mathbb{T}}^{*} \quad \forall \psi \in H_{\mathbb{T}}^{*}(M),
$$


where the sum is taken over all components $F$ of the fixed locus of $\mathbb{T}$. Part of the statement of (4.1) is that $\mathbf{e}(\mathcal{N} F)$ is invertible in $\mathcal{H}_{\mathbb{T}}^{*}(F)$.

The standard $\mathbb{T}$-action on $\mathbb{P}_{N}^{n-1}$ has $n N$ fixed points:

$$
P_{i_{1} \ldots i_{N}} \equiv P_{i_{1}} \times \ldots \times P_{i_{N}} .
$$

The restriction maps on the equivariant cohomology induced by the inclusions $P_{i_{1} \ldots i_{N}} \longrightarrow \mathbb{P}_{N}^{n-1}$ are the homomorphisms

$$
H_{\mathbb{T}}^{*}\left(\mathbb{P}_{N}^{n-1}\right) \longrightarrow \mathbb{Q}\left[\alpha_{1}, \ldots, \alpha_{n}\right], \quad \mathbf{x}_{s} \longrightarrow \alpha_{i_{s}}, s=1, \ldots, N
$$

By (3.1) and (4.2),

$$
\eta=\left.0 \in H_{\mathbb{T}}^{*}\left(\mathbb{P}_{N}^{n-1}\right) \quad \Longleftrightarrow \quad \eta\right|_{P_{i_{1} \ldots i_{N}}}=0 \in H_{\mathbb{T}}^{*} \quad \forall i_{s}=1,2, \ldots, n, s=1, \ldots, N,
$$

i.e. an element of $H_{\mathbb{T}}^{*}\left(\mathbb{P}_{N}^{n-1}\right)$ is determined by its restrictions to the $n N \mathbb{T}$-fixed points. For each $i=1,2, \ldots, n$, the equivariant Poincare dual of $P_{i}$ in $\mathbb{P}^{n-1}$ is given by

$$
\phi_{i}=\prod_{k \neq i}\left(\mathbf{x}-\alpha_{k}\right) \in H_{\mathbb{T}}^{*}\left(\mathbb{P}^{n-1}\right) .12
$$

Thus, by the defining property of the cohomology pushforward [27, (1.11)], the power series $\mathcal{Z}(\underline{\hbar}, \underline{\mathbf{x}}, Q)$ in (3.3) is completely determined by the $n N$ power series

$$
\mathcal{Z}\left(\underline{\hbar}, \alpha_{i_{1}, \ldots, i_{N}}, Q\right)=\sum_{d=0}^{\infty} Q^{d} \int_{\overline{\mathfrak{M}}_{0, N}\left(\mathbb{P}^{n-1}, d\right)} \mathbf{e}\left(\mathcal{V}_{d}\right) \prod_{s=1}^{s=N}\left(\frac{\mathrm{ev}_{s}^{*} \phi_{i_{s}}}{\hbar_{s}-\psi_{s}}\right)
$$

where $\alpha_{i_{1} \ldots i_{N}} \equiv\left(\alpha_{i_{1}}, \ldots, \alpha_{i_{N}}\right)$.

As described in detail in [15, Section 27.3], the fixed loci $\mathcal{Z}_{\Gamma}$ of the $\mathbb{T}$-action on $\overline{\mathfrak{M}}_{0, N}\left(\mathbb{P}^{n-1}, d\right)$ are indexed by $N$-marked decorated trees $\Gamma$. An $N$-marked decorated tree is a tuple

$$
\Gamma=(\operatorname{Ver}, \operatorname{Edg} ; \mu, \mathfrak{d}, \eta)
$$

where (Ver, Edg) is a tree and

$$
\mu: \text { Ver } \longrightarrow[n] \equiv\{1, \ldots, n\}, \quad \mathfrak{d}: \operatorname{Edg} \longrightarrow \mathbb{Z}^{+}, \quad \text { and } \quad \eta:[N] \longrightarrow \text { Ver }
$$

are maps such that

$$
\mu\left(v_{1}\right) \neq \mu\left(v_{2}\right) \quad \text { if } \quad\left\{v_{1}, v_{2}\right\} \in \text { Edg. }
$$

In the first diagram of Figure 2, the value of the map $\mu$ on each vertex is indicated by the number next to the vertex. Similarly, the value of the map $\mathfrak{d}$ on each edge is indicated by the number next to the edge. By (4.6), no two consecutive vertex labels are the same. Let

$$
|\Gamma|=\sum_{e \in \operatorname{Edg}} \mathfrak{d}(e) .
$$



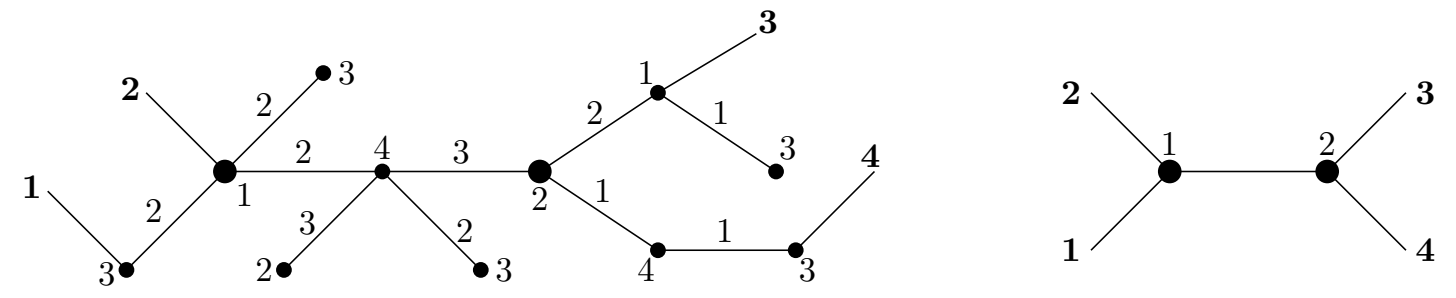

Figure 2: A decorated tree, with special vertices indicated by larger dots, and its decorated core

For each $e=\left\{v, v^{\prime}\right\} \in \mathrm{E}_{v}(\Gamma)$, let $\mu_{v}(e)=\mu\left(v^{\prime}\right) \in[n]$.

If $\Gamma$ is a decorated tree as in (4.5) and $v \in$ Ver, let

$$
\operatorname{val}_{\Gamma}(v)=\left|\mathrm{E}_{\Gamma}(v)\right|+\left|\eta^{-1}(v)\right|
$$

be the valence of $v$ in $\Gamma$. If in addition $N \geq 3$, the core of $\Gamma$ is the tuple $\bar{\Gamma} \equiv(\overline{\operatorname{Ver}}, \overline{\operatorname{Edg}} ; \bar{\mu}, \bar{\eta})$ such that

(R1) $(\overline{\mathrm{Ver}}, \overline{\mathrm{Edg}})$ is a tree, $\overline{\mathrm{Ver}}=\left\{v \in \operatorname{Ver}: \operatorname{val}_{\Gamma}(v) \geq 3\right\}$ and $\bar{\mu}=\left.\mu\right|_{\overline{\mathrm{Ver}}}$;

(R2) $\left\{v, v^{\prime}\right\} \in \overline{\operatorname{Edg}}$ if and only if $v, v^{\prime} \in \overline{\mathrm{Ver}}, v \neq v^{\prime}$, and for some $m \geq 0$ there exist distinct

$$
v_{1}, \ldots, v_{m} \in \operatorname{Ver}-\overline{\mathrm{Ver}} \quad \text { s.t. } \quad\left\{v_{i-1}, v_{i}\right\} \in \operatorname{Edg} \forall i \in[m+1], \quad \text { where } v_{0} \equiv v, v_{m+1} \equiv v^{\prime}
$$

(R3) if $s \in \eta^{-1}(\overline{\mathrm{Ver}}) \subset[N], \bar{\eta}(s)=\eta(s)$; if $s \in \eta^{-1}(\mathrm{Ver}-\overline{\mathrm{Ver}})$, there exist distinct elements

$$
v_{1}, \ldots, v_{m} \in \operatorname{Ver}-\overline{\mathrm{Ver}} \text { s.t. }\left\{v_{i-1}, v_{i}\right\} \in \operatorname{Edg} \forall i \in[m+1], \text { where } v_{0} \equiv \bar{\eta}(s), v_{m+1}=\eta(s) .
$$

The core of a graph with $N \geq 3$ is obtained by repeatedly collapsing all vertices with valence less than 3 onto their neighbors, until no such vertices are left; see Figure 2. We will call the vertices $\overline{\mathrm{Ver}}$ of the core $\bar{\Gamma}$ the special vertices of $\Gamma$.

The localization formula (4.1) reduces the restriction of (3.3) to each fixed point $P_{i_{1} \ldots i_{N}} \in \mathbb{P}_{N}^{n-1}$ to a sum over decorated trees. This sum can be computed by breaking each such tree $\Gamma$ at its special vertices into strands, with each of the strands keeping a copy of the special vertex, with its label, which will have a new marked point attached; see Figure 3. There are three types of strands:

(S1) one-marked strands;

(S2) strands with two new marked points;

(S3) strands with one new marked points and one of the original $N$ marked points.

\footnotetext{
${ }^{12}$ In other words, if $\eta \in H_{\mathbb{T}}^{*}\left(\mathbb{P}^{n-1}\right)$, then

$$
\left.\left.\eta\right|_{P_{i}} \equiv \int_{P_{i}} \eta\right|_{P_{i}}=\int_{\mathbb{P}^{n-1}} \eta \phi_{i} .
$$
}



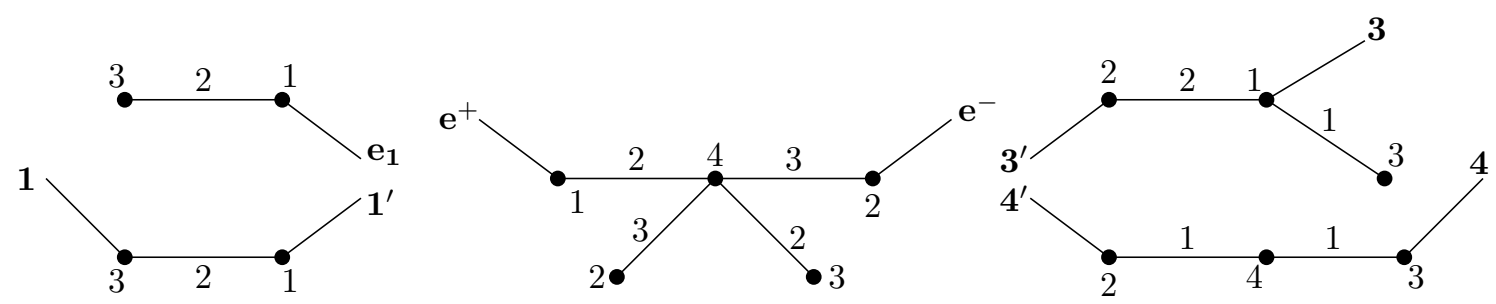

Figure 3: The strands of the graph in the first diagram in Figure 2.

By (4.1), each one-pointed strand at a special vertex $v \in \overline{\operatorname{Ver}} \subset$ Ver contributes to

$$
\mathcal{Z}^{\prime *}\left(\hbar, \alpha_{j}, Q\right) \equiv \sum_{d=1}^{\infty} Q^{d} \int_{\overline{\mathfrak{M}}_{0,1}\left(\mathbb{P}^{n-1}, d\right)} \mathbf{e}\left(\mathcal{V}_{d}^{\prime}\right) \frac{\mathrm{ev}_{1}^{*} \phi_{j}}{\hbar-\psi_{1}},
$$

where $j=\mu(v) \in[n]$ is the label of the vertex $v$ of $\Gamma$ and

$$
\mathcal{V}_{d}^{\prime} \longrightarrow \overline{\mathfrak{M}}_{0,1}\left(\mathbb{P}^{n-1}, d\right)
$$

is the kernel of the surjective vector bundle homomorphism $\widetilde{\mathrm{ev}}_{1}: \mathcal{V}_{d} \longrightarrow \mathrm{ev}_{1}^{*} \mathcal{L}$. By the dilaton relation [15, p527],

$$
\widetilde{\mathcal{Z}}^{*}\left(\hbar, \alpha_{j}, Q\right) \equiv \sum_{d=1}^{\infty} Q^{d} \int_{\overline{\mathfrak{M}}_{0,2}\left(\mathbb{P}^{n-1}, d\right)} \mathbf{e}\left(\mathcal{V}_{d}^{\prime}\right)\left(\frac{\mathrm{ev}_{1}^{*} \phi_{j}}{\hbar-\psi_{1}}\right)=\hbar^{-1} \mathcal{Z}^{\prime *}\left(\hbar, \alpha_{j}, Q\right) .
$$

Each of the two-pointed strands contributes to

$$
\mathcal{Z}^{*}\left(\hbar_{1}, \hbar_{2}, \alpha_{j_{1}}, \alpha_{j_{2}}, Q\right) \equiv \sum_{d=1}^{\infty} Q^{d} \int_{\overline{\mathfrak{M}}_{0,2}\left(\mathbb{P}^{n-1}, d\right)} \mathbf{e}\left(\mathcal{V}_{d}\right) \frac{\mathrm{ev}_{1}^{*} \phi_{j_{1}}}{\hbar_{1}-\psi_{1}} \frac{\mathrm{ev}_{2}^{*} \phi_{j_{2}}}{\hbar_{2}-\psi_{2}},
$$

where $j_{1}, j_{2} \in[n]$ are the labels of the vertices to which the marked points are attached. Thus, the power series $\mathcal{Z}(\underline{\hbar}, \underline{\mathbf{x}}, Q)$ in (3.3) is determined by the previously computed power series for one- and two-pointed GW-invariants.

While the number of one-marked strands at each node can be arbitrary large, as indicated in [27, Sections 2.1,2.2] it is possible to sum over all possibilities for these strands at each special vertex; see Corollary 4.3 below. On the other hand, the number of special vertices, the number of twopointed strands of type (S2), and the number of two-pointed strands of type (S3), are bounded (by $N-2, N-3$, and $N$, respectively). Using the Residue Theorem for $S^{2}$, one can then sum up over all possibilities of the markings for each of the distinguished nodes. Thus, the approach of breaking trees at special nodes reduces (3.3) to a finite sum, with one summand for each trivalent $N$-marked tree.

The description of the structure constants $c_{\mathbf{p}, \mathbf{b}}^{(d, t)}$ in Section 2.4 is obtained by breaking the trees at all special vertices. On the other hand, the description in Section 2.3 is obtained by breaking at the special vertex $\bar{\eta}(N)$ only. In addition to the strands (S1), we would then obtain strands with marked points indexed by the sets $S_{i} \sqcup\{0\}$, for a partition $\left\{S_{i}\right\}_{i \in[m]}$ of $[N]$ so that one of the sets $S_{i}$ is $\{N\}$. With either approach, the main step is summing over all possibilities for the strands (S1), as done in Corollary 4.3 . 


\subsection{Notation and preliminaries}

If $f=f(\hbar)$ is a rational function in $\hbar$ and $\hbar_{0} \in S^{2}$, let

$$
\underset{\hbar=\hbar_{0}}{\Re}\{f(\hbar)\}=\frac{1}{2 \pi \mathfrak{i}} \oint f(\hbar) \mathrm{d} \hbar,
$$

where the integral is taken over a positively oriented loop around $\hbar=\hbar_{0}$ containing no other singular points of $f$, denote the residue of $f(\hbar) \mathrm{d} \hbar$ at $\hbar=\hbar_{0}$. With this definition,

$$
\underset{\hbar=\infty}{\mathfrak{R}}\{f(\hbar)\}=-\underset{w=0}{\mathfrak{R}}\left\{w^{-2} f\left(w^{-1}\right)\right\} .
$$

If $f$ involves variables other than $\hbar, \underset{\hbar=\hbar_{0}}{\mathfrak{R}}\{f(\hbar)\}$ will be a function of such variables. If $f$ is a power series in $q$ with coefficients that are rational functions in $\hbar$ and possibly other variables, denote by $\underset{\hbar=\hbar_{0}}{\mathfrak{R}}\{f(\hbar)\}$ the power series in $q$ obtained by replacing each of the coefficients by its residue at $\hbar=\hbar_{0}$. If $\hbar_{1}, \ldots, \hbar_{k}$ is a collection of distinct points in $S^{2}$, let

$$
\underset{\hbar=\hbar_{1}, \ldots, \hbar_{k}}{\mathfrak{R}}\{f(\hbar)\}=\sum_{i=1}^{i=k} \underset{\hbar=\hbar_{i}}{\mathfrak{R}}\{f(\hbar)\}
$$

be the sum of the residues at the specified values of $\hbar$.

We denote by

$$
\mathbb{Q}_{\alpha}^{\prime} \equiv \mathbb{Q}\left[\alpha, \sigma_{n}^{-1}, D_{\alpha}^{-1}\right] \subset \mathbb{Q}_{\alpha}
$$

the subring of rational functions in $\alpha_{1}, \ldots, \alpha_{n}$ with denominators that are products of $\sigma_{n}$ and $D_{\alpha}$. Let

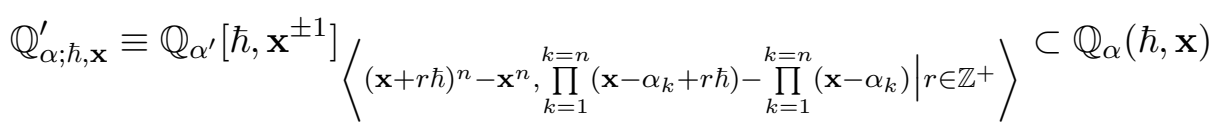

be the subring of rational functions in $\alpha_{1}, \ldots, \alpha_{n}, \hbar$, and $\mathbf{x}$ with numerators that are polynomials in $\alpha_{1}, \ldots, \alpha_{n}, \hbar$, and $\mathbf{x}$ and with denominators that are products of

$$
\sigma_{n}, \quad D_{\alpha}, \quad \mathbf{x}, \quad(\mathbf{x}+r \hbar)^{n}-\mathbf{x}^{n}, \quad \prod_{k=1}^{k=n}\left(\mathbf{x}-\alpha_{k}+r \hbar\right)-\prod_{k=1}^{k=n}\left(\mathbf{x}-\alpha_{k}\right), \quad \text { with } r \in \mathbb{Z}^{+} .
$$

If $R$ is one of the rings $\mathbb{Q}_{\alpha}^{\prime}, \mathbb{Q}_{\alpha}^{\prime}\left[\mathbf{x}^{ \pm 1}\right]$, or $\mathbb{Q}_{\alpha ; \hbar, \mathbf{x}}^{\prime}$ and $f_{1}$ and $f_{2}$ are elements of $R$ or $R[[Q]]$, we will write $f_{1} \sim f_{2}$ if $f_{1} \sim f_{2}$ lies in $\mathcal{I} \cdot R$ or $\mathcal{I} \cdot R[[Q]]$, respectively. By the next lemma, certain operations on these rings respect these equivalence relations.

Lemma 4.1. (1) If $f \in \mathbb{Q}_{\alpha ; \hbar, \mathbf{x}}^{\prime}$, there exists $g \in \mathbb{Q}_{\alpha}^{\prime}\left[\mathbf{x}^{ \pm 1}\right]$ such that

$$
\underset{\hbar=0}{\mathfrak{R}}\left\{f\left(\hbar, \mathbf{x}=\alpha_{j}\right)\right\}=g\left(\mathbf{x}=\alpha_{j}\right) \quad \forall j \in[n] .
$$

(2) If $g \in \mathbb{Q}_{\alpha}^{\prime}\left[\mathbf{x}^{ \pm 1}\right]$,

$$
\underset{\mathbf{x}=0, \infty}{\mathfrak{R}}\left\{\frac{g(\mathbf{x})}{\prod_{k=1}^{k=n}\left(\mathbf{x}-\alpha_{k}\right)}\right\} \in \mathbb{Q}_{\alpha}^{\prime} .
$$


(3) For every $p \in \mathbb{Z}$,

$$
-\underset{\mathbf{x}=0, \infty}{\mathfrak{R}}\left\{\frac{\mathbf{x}^{p}}{\prod_{k=1}^{k=n}\left(\mathbf{x}-\alpha_{k}\right)}\right\} \sim \begin{cases}\hat{\sigma}_{n}^{t}, & \text { if } p=n-1+n t \text { with } t \in \mathbb{Z} ; \\ 0, & \text { if } p+1 \notin n \mathbb{Z} .\end{cases}
$$

Proof. If $f \in \mathbb{Q}_{\alpha ; \hbar, \mathbf{x}}^{\prime}$, then

$$
\begin{gathered}
\underset{\hbar=0}{\Re}\left\{f\left(\hbar, \mathbf{x}=\alpha_{j}\right)\right\}=\left.(\underset{\hbar=0}{\Re}\{f(\hbar, \mathbf{x})\})\right|_{\mathbf{x}=\alpha_{j}}, \quad \underset{\hbar=0}{\mathfrak{R}}\{f(\hbar, \mathbf{x})\} \in \mathbb{Q}_{\alpha}^{\prime}\left[\mathbf{x}^{ \pm 1}, \sigma_{n-1}(\mathbf{x})^{-1}\right], \\
\text { where } \quad \sigma_{n-1}(\mathbf{x})=\sum_{i=1}^{i=n} \prod_{k \neq i}\left(\mathbf{x}-\alpha_{k}\right) .
\end{gathered}
$$

The first claim of this lemma thus follows from the observation that

$$
\left.\frac{1}{\sigma_{n-1}(\mathbf{x})}\right|_{\mathbf{x}=\alpha_{j}}=\left.\frac{1}{D_{\alpha}^{2}}\left(\sum_{i=1}^{i=n}\left(\prod_{\substack{i^{\prime} \neq i \\ k \neq i^{\prime}}}\left(\alpha_{i^{\prime}}-\alpha_{k}\right)^{2}\right)\left(\prod_{k \neq i}\left(\mathbf{x}-\alpha_{k}\right)\right)\right)\right|_{\mathbf{x}=\alpha_{j}} \quad \forall j \in[n] .
$$

The second claim is immediate from the third. The third claim of this lemma follows from the power series expansions

$$
-\frac{1}{\mathbf{x}^{n}-\hat{\sigma}_{n}}=\sum_{r=0}^{\infty} \hat{\sigma}_{n}^{-r-1} \mathbf{x}^{n r}, \quad \frac{1}{1-\hat{\sigma}_{n} w^{n}}=\sum_{r=0}^{\infty} \hat{\sigma}_{n}^{r} w^{n r} .
$$

around $\mathbf{x}=0$ and $w=0$, respectively.

We will also use the Residue Theorem on $S^{2}$ :

$$
\sum_{\mathbf{x}_{0} \in S^{2}} \underset{\mathbf{x}=\mathbf{x}_{0}}{\mathfrak{R}}\{f(\mathbf{x})\}=0
$$

for every rational function $f=f(\mathbf{x})$ on $S^{2} \supset \mathbb{C}$.

\subsection{Equivariant one- and two-pointed formulas}

The most fundamental generating function for GW-invariants in the mirror symmetry computations following [10] is

$$
\begin{aligned}
\widetilde{\mathcal{Z}}(\hbar, \mathbf{x}, Q) & \equiv 1+\widetilde{\mathcal{Z}}^{*}(\hbar, \mathbf{x}, Q) \\
& \equiv 1+\sum_{d=1}^{\infty} Q^{d} \operatorname{ev}_{1 *}\left\{\frac{\mathbf{e}\left(\mathcal{V}_{d}^{\prime}\right)}{\hbar-\psi_{1}}\right\} \in H_{\mathbb{T}}^{*}\left(\mathbb{P}^{n-1}\right)\left[\left[\hbar^{-1}, Q\right]\right]
\end{aligned}
$$

where $e_{1}: \overline{\mathfrak{M}}_{0,2}\left(\mathbb{P}^{n-1}, d\right) \longrightarrow \mathbb{P}^{n-1}$ and $\mathcal{V}_{d}^{\prime} \longrightarrow \overline{\mathfrak{M}}_{0,2}\left(\mathbb{P}^{n-1}, d\right)$ is the kernel of the surjective vector bundle homomorphism $\widetilde{e v}_{1}: \mathcal{V}_{d} \longrightarrow \operatorname{ev}_{1}^{*} \mathcal{L}$. By [10], $\widetilde{\mathcal{Z}}\left(\hbar, \alpha_{j}, Q\right) \in \mathbb{Q}_{\alpha}(\hbar)$ for $j \in[n]$. Thus, we can define

$$
\begin{aligned}
\zeta\left(\alpha_{j}, Q\right) & =\underset{\hbar=0}{\Re}\left\{\ln \left(1+\widetilde{\mathcal{Z}}^{*}\left(\hbar, \alpha_{j}, Q\right)\right)\right\} \in Q \cdot \mathbb{Q}_{\alpha}[[Q]], \\
\widetilde{\mathcal{Z}}_{m, B}\left(\alpha_{j}, Q\right) & =\sum_{m^{\prime}=0}^{\infty} \frac{\left(m^{\prime}+m\right) !}{m^{\prime} !} \sum_{\mathbf{b} \in \mathcal{P}_{m^{\prime}}\left(m-B+m^{\prime}\right)}\left(\prod_{k=1}^{k=m^{\prime}} \frac{(-1)^{b_{k}}}{b_{k} !} \underset{\hbar=0}{\Re}\left\{\hbar^{-b_{k}} \widetilde{\mathcal{Z}}^{*}\left(\hbar, \alpha_{j}, Q\right)\right\}\right) \in \mathbb{Q}_{\alpha}[[Q]],
\end{aligned}
$$


for $m, B \in \overline{\mathbb{Z}}^{+}$. Since the power series $\widetilde{\mathcal{Z}}^{*}(\hbar, \mathbf{x}, Q)$ has no $Q$-constant term, the above sum is finite in each $Q$-degree. It is shown Section 4.4 that the power series $\widetilde{\mathcal{Z}}_{m, B}(\mathbf{x}, Q)$ describe the contributions of the strands (S1) at a vertex $v$ of the core of a tree with $m_{v}=m$ (with $m_{v}$ computed with respect to the core). Let

$$
\begin{aligned}
\mathcal{Z}^{*}\left(\hbar_{1}, \hbar_{2}, \mathbf{x}_{1}, \mathbf{x}_{2}, Q\right) & =\sum_{d=1}^{\infty} Q^{d} \operatorname{ev}_{*}\left\{\frac{\mathbf{e}(\mathcal{V})}{\left(\hbar_{1}-\psi_{1}\right)\left(\hbar_{2}-\psi_{2}\right)}\right\} \in H_{\mathbb{T}}^{*}\left(\mathbb{P}_{2}^{n-1}\right)\left[\left[\hbar_{1}^{-1}, \hbar_{2}^{-1}, Q\right]\right], \\
\mathcal{Z}\left(\hbar_{1}, \hbar_{2}, \mathbf{x}_{1}, \mathbf{x}_{2}, Q\right) & =-\frac{\langle\mathbf{a}\rangle \mathbf{x}_{1}^{l}}{\hbar_{1}+\hbar_{2}} \sum_{\substack{p_{1}+p_{2}+r=n-1 \\
p_{1}, p_{2}, r \geq 0}} \hat{\sigma}_{r} \mathbf{x}_{1}^{p_{1}} \mathbf{x}_{2}^{p_{2}}+\mathcal{Z}^{*}\left(\hbar_{1}, \hbar_{2}, \mathbf{x}_{1}, \mathbf{x}_{2}, Q\right),
\end{aligned}
$$

where ev: $\overline{\mathfrak{M}}_{0,2}\left(\mathbb{P}^{n-1}, d\right) \longrightarrow \mathbb{P}_{2}^{n-1}$ is the total evaluation map.

Proposition 4.2. The power series (4.9) and (3.4) admit expansions

$$
\begin{aligned}
\widetilde{\mathcal{Z}}\left(\hbar, \alpha_{j}, Q\right) & =e^{\zeta\left(\alpha_{j}, Q\right) / \hbar} \sum_{b=0}^{\infty} \Psi_{b}\left(\alpha_{j}, Q\right) \hbar^{b}, \\
\frac{\mathcal{Z}_{p}\left(\hbar, \alpha_{j}, Q\right)}{n-l-1} & =e^{\zeta\left(\alpha_{j}, Q\right) / \hbar} \sum_{b=0}^{\infty} \Psi_{p ; b}\left(\alpha_{j}, Q\right) \hbar^{b}, \\
\prod_{r=p-l+1}^{\infty} I_{r}(q) &
\end{aligned}
$$

for some $\zeta, \Psi_{b}, \Psi_{p ; b} \in \mathbb{Q}_{\alpha}^{\prime}\left[\mathbf{x}^{ \pm 1}\right][[Q]]$ such that

$$
\Psi_{b}(\mathbf{x}, Q) \sim \frac{\Phi_{b}(\mathbf{q})}{I_{0}(\mathbf{q})} \mathbf{x}^{-b}, \quad \Psi_{p ; b}(\mathbf{x}, Q) \sim \frac{I_{0}(\mathbf{q}) \Phi_{p ; b}(\mathbf{q})}{L(\mathbf{q})^{\delta_{0 \nu_{\mathbf{a}}}} n} \mathbf{x}^{p-b}
$$

where $\mathbf{q} e^{\delta_{0 \nu_{\mathbf{a}}} J(\mathbf{q})}=Q / \mathbf{x}^{\nu_{\mathbf{a}}}$.

Proof. The existence of the expansion (4.11) follows from Lemmas 2.2 and 2.3 in [27, but a direct argument is provided below and in Appendix A, Let

$$
\mathcal{Y}(\hbar, \mathbf{x}, q)=\sum_{d=0}^{\infty} q^{d} \frac{\prod_{k=1}^{k=l} \prod_{r=1}^{r=a_{k} d}\left(a_{k} \mathbf{x}+r \hbar\right)}{\prod_{r=1}^{r=d}\left(\prod_{k=1}^{k=n}\left(\mathbf{x}-\alpha_{k}+r \hbar\right)-\prod_{k=1}^{k=n}\left(\mathbf{x}-\alpha_{k}\right)\right)} \in\left(\mathbb{Q}_{\alpha ; \hbar, \mathbf{x}}^{\prime} \cap \mathbb{Q}_{\alpha}[\mathbf{x}]\left[\left[\hbar^{-1}\right]\right]\right)[[Q]] .
$$

By [15, Section 29.1],

$$
\widetilde{\mathcal{Z}}(\hbar, \mathbf{x}, Q)=e^{-J(q) \frac{\mathbf{x}^{\delta} 0 \nu_{\mathbf{a}}}{\hbar}+f(q) \frac{\sigma_{1}}{\hbar}} \frac{\mathcal{Y}(\hbar, \mathbf{x}, q)}{I_{0}(q)}
$$

for some $f \in q \mathbb{Q}[[q]]$ (which is 0 unless $\nu_{\mathbf{a}}=0$ ), where $q e^{\delta_{0 \nu_{\mathbf{a}}} J(q)}=Q$. Since

$$
\mathcal{Y}(\hbar, \mathbf{x}, q)=\left\{1+\frac{\hbar}{\mathbf{x}} q \frac{\mathrm{d}}{\mathrm{d} q}\right\}^{l} \mathcal{Y}_{0}(\hbar, \mathbf{x}, q),
$$

with $\mathcal{Y}_{0}(\hbar, \mathbf{x}, q)$ given by (A.1), Lemma A.1 implies that $\mathcal{Y}(\hbar, \mathbf{x}, q)$ admits an expansion of the form

$$
\mathcal{Y}(\hbar, \mathbf{x}, q)=e^{\xi(\mathbf{x}, q) / \hbar} \sum_{b=0}^{\infty} \Phi_{b}(\mathbf{x}, q) \hbar^{b}
$$


with $\xi(\mathbf{x}, q), \Phi_{0}(\mathbf{x}, q), \Phi_{1}(\mathbf{x}, q), \ldots \in \mathbb{Q}_{\alpha}(\mathbf{x})[[q]]$. Since

$$
\begin{gathered}
\xi(\mathbf{x}, q)=\underset{\hbar=0}{\mathfrak{R}}\{\ln \mathcal{Y}(\hbar, \mathbf{x}, q)\}, \quad \Phi_{b}(\mathbf{x}, q)=\underset{\hbar=0}{\mathfrak{R}}\left\{\hbar^{-b-1} e^{-\xi(\mathbf{x}, q) / \hbar} \mathcal{Y}(\hbar, \mathbf{x}, q)\right\}, \\
\text { and } \mathcal{Y}(\hbar, \mathbf{x}, q)-F(w, \mathbf{q}) \in q \cdot \mathcal{I} \mathbb{Q}_{\alpha ; \hbar, \mathbf{x}}^{\prime},
\end{gathered}
$$

where $w=\mathbf{x} / \hbar$, Proposition 2.1 and the first statement of Lemma 4.1 imply that there exist

$$
\tilde{\xi}(\mathbf{x}, q), \tilde{\Phi}_{0}(\mathbf{x}, q), \tilde{\Phi}_{1}(\mathbf{x}, q), \ldots \in \mathbb{Q}_{\alpha}^{\prime}\left[\mathbf{x}^{ \pm 1}\right][[q]]
$$

such that

$$
\begin{aligned}
\mathcal{Y}\left(\hbar, \alpha_{j}, q\right) & =e^{\tilde{\xi}\left(\alpha_{j}, q\right) / \hbar} \sum_{b=0}^{\infty} \tilde{\Phi}_{b}\left(\alpha_{j}, q\right) \hbar^{b} \quad \forall j \in[n], \\
\tilde{\xi}(\mathbf{x}, q) & \sim \xi(\mathbf{q}) \mathbf{x}, \quad \tilde{\Phi}_{b}(\mathbf{x}, q) \sim \Phi_{b}(\mathbf{q}) \mathbf{x}^{-b} \quad \forall b \in \mathbb{Z}^{+} .
\end{aligned}
$$

By (4.14) and (4.16), (4.11) and the first statement in (4.13) hold with

$$
\zeta(\mathbf{x}, Q)=\tilde{\xi}(\mathbf{x}, q)-J(\mathbf{q}) \mathbf{x}+f(q) \sigma_{1}, \quad \Psi_{b}(\mathbf{x}, Q)=\frac{\tilde{\Phi}_{b}(\mathbf{x}, \mathbf{q})}{I_{0}(q)}=\frac{\tilde{\Phi}_{b}(\mathbf{x}, \mathbf{q})}{I_{0}(\mathbf{q})}
$$

The existence of the expansion (4.12) follows from the existence of the expansion (4.11) and the description of $\mathcal{Z}_{p}(\hbar, \mathbf{x}, Q)$ as a linear combination of the derivatives of $\widetilde{\mathcal{Z}}(\hbar, \mathbf{x}, Q)$ in [24, Theorem 4]. By [24, Theorem 4],

$$
\mathcal{Z}_{p}(\hbar, \mathbf{x}, Q) \sim e^{-J(\mathbf{q}) w} \mathbf{x}^{p} \frac{F_{p}(w, \mathbf{q})}{I_{p-l}(\mathbf{q})} .
$$

Along with the first statement in Lemma 4.1 and (2.21), this gives the second claim in (4.13).

Corollary 4.3. For all $m \in \overline{\mathbb{Z}}^{+}$and $\mathbf{c} \in\left(\overline{\mathbb{Z}}^{+}\right)^{\infty}$, there exists $\Psi_{m, \mathbf{c}} \in \mathbb{Q}_{\alpha}^{\prime}\left[\mathbf{x}^{ \pm 1}\right][[Q]]$ such that

$$
\widetilde{\mathcal{Z}}_{m, B}\left(\alpha_{j}, Q\right)=\sum_{\mathbf{c} \in\left(\overline{\mathbb{Z}}^{+}\right) \infty}\left((-1)^{m-\|\mathbf{c}\|}\left(\begin{array}{c}
B \\
m-\|\mathbf{c}\|
\end{array}\right) \zeta\left(\alpha_{j}, Q\right)^{B-(m-\|\mathbf{c}\|)} \Psi_{m, \mathbf{c}}\left(\alpha_{j}, Q\right)\right)
$$

for all $B \in \overline{\mathbb{Z}}^{+}$and $j \in[n]$ and

$$
\Psi_{m, \mathbf{c}}(\mathbf{x}, Q) \sim\left(\frac{I_{0}(\mathbf{q})}{\Phi_{0}(\mathbf{q})}\right)^{m+3} \Phi_{m, \mathbf{c}}(\mathbf{q}) \mathbf{x}^{-\|\mathbf{c}\|},
$$

where $\mathbf{q} e^{\delta_{0 \nu_{\mathbf{a}}} J(\mathbf{q})}=Q / \mathbf{x}^{\nu_{\mathbf{a}}}$.

Proof. By Lemma B.2 and (4.11), (4.17) holds with

$$
\Psi_{m, \mathbf{c}}(\mathbf{x}, Q)=(-1)^{m+|\mathbf{c}|}(m+|\mathbf{c}|) ! \frac{1}{\Psi_{0}(\mathbf{x}, Q)^{m+1}} \prod_{r=1}^{\infty} \frac{1}{c_{r} !}\left(\frac{1}{(r+1) !} \frac{\Psi_{r}(\mathbf{x}, Q)}{\Psi_{0}(\mathbf{x}, Q)}\right)^{c_{r}} .
$$

Along with the first statement in (4.13) and (2.30), this implies (4.18). 
Lemma 4.4. There exists a collection $\left\{\mathcal{C}_{p_{-} p_{+}}\right\}_{p_{ \pm} \in \llbracket n \Downarrow} \subset \mathbb{Q}[\alpha][[Q]]$ such that

$$
\begin{aligned}
\frac{1}{\langle\mathbf{a}\rangle} \underset{\hbar_{+}=0}{\mathfrak{R}}\left\{\frac{1}{\hbar_{+}^{1+b_{+}}} e^{-\frac{\zeta\left(\alpha_{j_{+}}, Q\right)}{\hbar_{+}}} \mathcal{Z}\left(\hbar_{-}, \hbar_{+}, \alpha_{j_{-}}, \alpha_{j_{+}}, Q\right)\right\} \\
\quad=\sum_{b_{-}=0}^{b_{-}=b_{+}}\left(\frac{(-1)^{b_{-}}}{\hbar_{-}^{b_{-}}} \sum_{p_{+}, p_{-} \in\lfloor n \rrbracket} \mathcal{C}_{p_{-} p_{+}}(Q) \Psi_{p_{+} ; b_{+}-b_{-}}\left(\alpha_{j_{+}}, Q\right) \frac{\mathcal{Z}_{p_{-}}\left(\hbar_{-}, \alpha_{j_{-}}, Q\right)}{\hbar_{-} \prod_{r=p_{-}-l+1}^{n-l-1} I_{r}(q)}\right)
\end{aligned}
$$

for all $b_{+} \in \overline{\mathbb{Z}}^{+}$and $j_{-}, j_{+} \in[n]$ and

$$
\mathcal{C}_{p_{-} p_{+}}(Q) \sim \begin{cases}\frac{L(q)^{\delta} 0 \nu_{\mathbf{a}}(1+t) n}{I_{0}(q)^{2}} \hat{\sigma}_{n}^{t}, & \text { if } p_{-}+p_{+}+n t=n-1+l, t=0,1, \\ 0, & \text { otherwise }\end{cases}
$$

where $q e^{\delta_{0 \nu_{\mathbf{a}}} J(q)}=Q$.

Proof. By [24, Theorem 4],

$$
\begin{aligned}
& \mathcal{Z}\left(\hbar_{-}, \hbar_{+}, \mathbf{x}_{-}, \mathbf{x}_{+}, Q\right) \\
& =\frac{\langle\mathbf{a}\rangle}{\hbar_{-}+\hbar_{+}}\left\{\sum_{\substack{p_{-}+p_{+}+r=n-1+l \\
p_{-}, p_{+} \in \llbracket n \rrbracket, r \in \overline{\mathbb{Z}}^{+} \\
p_{-}, p_{+} \geq l}}+\sum_{\begin{array}{c}
p_{-}+p_{+}+r=n-1+l \\
p_{-}, p_{+} \in\left\lfloor n \rrbracket, r \in \overline{\mathbb{Z}}^{+}\right. \\
p_{-}, p_{+}<l
\end{array}}\right\} \hat{\sigma}_{r} \mathcal{Z}_{p_{-}}\left(\hbar_{-}, \mathbf{x}_{-}, Q\right) \mathcal{Z}_{p_{+}}\left(\hbar_{+}, \mathbf{x}_{+}, Q\right) .
\end{aligned}
$$

Combining this identity with (4.12), we find that (4.20) holds with

$$
\mathcal{C}_{p_{-} p_{+}}(Q)=\left(\prod_{r=p_{+}-l+1}^{n-l-1} I_{r}(q)\right)\left(\prod_{r=p_{-}-l+1}^{n-l-1} I_{r}(q)\right) \hat{\sigma}_{n-1+l-p_{-}-p_{+}} \cdot \begin{cases}1, & \text { if } p_{-}, p_{+}<l \\ -1, & \text { if } p_{-}, p_{+} \geq l \\ 0, & \text { otherwise }\end{cases}
$$

Along with the first two statements in Lemma 2.3, this implies (4.21).

\subsection{Main localization computation}

We now prove Theorem $\mathbf{B}$, with each of the two definitions of the structure constants $\mathbf{c}_{\mathbf{p}, \mathbf{b}}^{(d, t)}$, by summing up the contributions of the $\mathbb{T}$-fixed loci $\mathcal{Z}_{\Gamma}$ of $\overline{\mathfrak{M}}_{0, N}\left(\mathbb{P}^{n-1}, d\right)$, with $d \in \overline{\mathbb{Z}}^{+}$. As outlined in Section 4.1, this will be done by breaking each $\Gamma$ (and correspondingly each fixed locus $\mathcal{Z}_{\Gamma}$ ) at either one special vertex, $v=\bar{\mu}(N)$, or at every special vertex of $\Gamma$.

Let $\Gamma$ be a decorated tree with $N$ marked points as in (4.5). Let $\bar{\Gamma} \equiv(\overline{\operatorname{Ver}}, \overline{\operatorname{Edg}} ; \bar{\mu}, \bar{\eta})$ be the core of $\Gamma$ as in Section 4.1 and $v=\bar{\eta}(N)$. Similarly to Figure 3, we break $\Gamma$ at the vertex $v \in \overline{\operatorname{Ver}} \subset$ Ver into strands $\Gamma_{e}$ indexed by the set $\mathrm{E}_{v}(\Gamma)$ of the edges with vertex $v$ in $\Gamma$; each strand $\Gamma_{e}$ keeps a copy of the vertex $v$ and gains an extra marked point, which will be labeled $e$, attached at $v$. For each $e \in \mathrm{E}_{v}(\Gamma)$, denote by $S_{e} \subset[N]$ the subset of the original marked points carried by the strand $\Gamma_{e}$. Let

$$
\begin{aligned}
& \mathrm{E}_{v}^{*}(\Gamma)=\left\{e \in \mathrm{E}_{v}(\Gamma): S_{e} \neq \emptyset\right\} \sqcup \eta^{-1}(v), \quad \mathrm{E}_{v}^{\prime}(\Gamma)=\left\{e \in \mathrm{E}_{v}(\Gamma): S_{e}=\emptyset\right\}, \\
& \overline{\mathrm{E}}_{v}(\Gamma)=\mathrm{E}_{v}^{*}(\Gamma) \cup \mathrm{E}_{v}^{\prime}(\Gamma) \subset \mathrm{E}_{v}(\Gamma) \sqcup[N] .
\end{aligned}
$$


Thus, $\left|\mathrm{E}_{v}^{\prime}(\Gamma)\right| \geq 0,\left|\mathrm{E}_{v}^{*}(\Gamma)\right| \geq 3$ (because $\bar{\Gamma}$ is a trivalent tree), and $\left\{S_{e}\right\}_{e \in \mathrm{E}_{v}^{*}(\Gamma)} \in \mathcal{P}_{\mathrm{E}_{v}^{*}(\Gamma)}([N])$, where $S_{e} \equiv\{e\}$ if $e \in \eta^{-1}(v)$.

The fixed locus $\mathcal{Z}_{\Gamma}$ corresponding to $\Gamma$, the restriction of $\mathbf{e}(\mathcal{V})$ to $\mathcal{Z}_{\Gamma}$, and the euler class of the normal bundle of $\mathcal{Z}_{\Gamma}$ are given by

$$
\begin{aligned}
& \mathcal{Z}_{\Gamma}=\overline{\mathcal{M}}_{0, \overline{\mathrm{E}}_{v}(\Gamma)} \times \prod_{e \in \mathrm{E}_{v}(\Gamma)} \mathcal{Z}_{\Gamma_{e}}, \quad \frac{\mathbf{e}(\mathcal{V})}{\mathbf{e}\left(\mathcal{L}_{\mu(v)}\right)}=\prod_{e \in \mathrm{E}_{v}(\Gamma)} \frac{\pi_{e}^{*} \mathbf{e}(\mathcal{V})}{\mathbf{e}\left(\mathcal{L}_{\mu(v)}\right)} \\
& \frac{\mathbf{e}\left(T_{\mu(v)} \mathbb{P}^{n-1}\right)}{\mathbf{e}\left(\mathcal{N} \mathcal{Z}_{\Gamma}\right)}=\prod_{e \in \mathrm{E}_{v}(\Gamma)} \frac{\mathbf{e}\left(T_{\mu(v)} \mathbb{P}^{n-1}\right)}{\mathbf{e}\left(\mathcal{N} \mathcal{Z}_{\Gamma_{e}}\right)\left(\hbar_{e}^{\prime}-\pi_{e}^{*} \psi_{e}\right)}
\end{aligned}
$$

where $\overline{\mathcal{M}}_{0, \mathrm{E}_{v}(\Gamma)} \approx \overline{\mathcal{M}}_{0,\left|\mathrm{E}_{v}(\Gamma)\right|+\left|\eta^{-1}(v)\right|}$ is the moduli space of stable rational $\overline{\mathrm{E}}_{v}(\Gamma)$-marked curves,

$$
\hbar_{e}^{\prime} \equiv c_{1}\left(L_{e}^{\prime}\right) \in H^{*}\left(\overline{\mathcal{M}}_{0, \overline{\mathrm{E}}_{v}(\Gamma)}\right)
$$

is the first chern class of the universal tangent line bundle for the marked point corresponding to the edge $e$, and

$$
\pi_{e}: \mathcal{Z}_{\Gamma} \longrightarrow \mathcal{Z}_{\Gamma_{e}} \subset \bigcup_{d_{e}=1}^{\infty} \overline{\mathfrak{M}}_{0, S_{e} \sqcup\{e\}}\left(\mathbb{P}^{n-1}, d_{e}\right)
$$

is the projection map. By [15, Section 27.2],

$$
\psi_{e} \mid \mathcal{Z}_{\Gamma_{e}}=\frac{\alpha_{\mu_{v}(e)}-\alpha_{\mu(v)}}{\mathfrak{d}(e)} .
$$

Thus, by [15, Exercise 25.2.8],

$$
\begin{aligned}
& \int_{\overline{\mathcal{M}}_{0, \mathrm{E}_{v}(\Gamma)}}\left\{\left(\prod_{e \in \mathrm{E}_{v}(\Gamma)} \frac{1}{\hbar_{e}^{\prime}-\pi_{e}^{*} \psi_{e}}\right)\left(\prod_{e \in \eta^{-1}(v)} \frac{1}{\hbar_{e}-\psi_{e}}\right)\right\} \\
& =(-1)^{\left|\mathrm{E}_{v}(\Gamma)\right|} \sum_{\mathbf{b} \in\left(\overline{\mathbb{Z}}^{+}\right)^{\mathrm{E}_{v}(\Gamma)}} \int_{\overline{\mathcal{M}}_{0, \overline{\mathrm{E}}_{v}(\Gamma)}}\left\{\left(\prod_{e \in \mathrm{E}_{v}(\Gamma)} \psi_{e}^{-b_{e}-1} \hbar_{e}^{\prime b_{e}}\right)\left(\prod_{e \in \eta^{-1}(v)} \hbar_{e}^{-b_{e}-1} \psi_{e}^{b_{e}}\right)\right\} \\
& =\sum_{\mathbf{b} \in\left(\overline{\mathbb{Z}}^{+}\right)^{\overline{\mathrm{E}}_{v}(\Gamma)}}\left\{\left(\begin{array}{c}
\left|\overline{\mathrm{E}}_{v}(\Gamma)\right|-3 \\
\mathbf{b}
\end{array}\right)\left(\prod_{e \in \mathrm{E}_{v}(\Gamma)}\left(\frac{\alpha_{\mu(v)}-\alpha_{\mu_{v}(e)}}{\mathfrak{d}(e)}\right)^{-b_{e}-1}\right)\left(\prod_{e \in \eta^{-1}(v)} \hbar_{e}^{-b_{e}-1}\right)\right\} .
\end{aligned}
$$

Combining this with (4.24), (3.2), and (4.3), we obtain

$$
\begin{aligned}
& \frac{\prod_{k \neq \mu(v)}\left(\alpha_{\mu(v)}-\alpha_{k}\right)}{\langle\mathbf{a}\rangle \alpha_{\mu(v)}^{l}} \int_{\mathcal{Z}_{\Gamma}} \frac{\mathbf{e}(\mathcal{V})}{\mathbf{e}\left(\mathcal{N} \mathcal{Z}_{\Gamma}\right)} \prod_{s=1}^{s=N}\left(\frac{\mathrm{ev}_{s}^{*} \phi_{i_{s}}}{\hbar_{s}-\psi_{s}}\right) \\
& =\sum_{\mathbf{b} \in\left(\overline{\mathbb{Z}}^{+}\right)^{\overline{\mathrm{E}}_{v}(\Gamma)}}\left\{\left(\begin{array}{c}
\left|\overline{\mathrm{E}}_{v}(\Gamma)\right|-3 \\
\mathbf{b}
\end{array}\right) \prod_{s \in \eta^{-1}(v)}\left(\hbar_{s}^{-b_{s}-1} \prod_{k \neq i_{s}}\left(\alpha_{\mu(v)}-\alpha_{k}\right)\right)\right. \\
& \left.\times \prod_{e \in \mathrm{E}_{v}(\Gamma)}\left(\left(\frac{\alpha_{\mu(v)}-\alpha_{\mu_{v}(e)}}{\mathfrak{d}(e)}\right)^{-b_{e}-1} \int_{\mathcal{Z}_{\Gamma_{e}}} \frac{\mathbf{e}(\mathcal{V}) \mathrm{ev}_{e}^{*} \phi_{\mu(v)}}{\langle\mathbf{a}\rangle \alpha_{\mu(v)}^{l} \mathbf{e}\left(\mathcal{N} \mathcal{Z}_{\Gamma_{e}}\right)} \prod_{s \in S_{e}}\left(\frac{\mathrm{ev}_{s}^{*} \phi_{i_{s}}}{\hbar_{s}-\psi_{s}}\right)\right)\right\} .
\end{aligned}
$$


The equality holds after dividing the right-hand side by the order of the appropriate group of symmetries; see [15, Section 27.3]. This group is taken into account in the next paragraph.

We now sum up (4.26) over all possibilities for $\Gamma$. If $e \in \mathrm{E}_{v}^{\prime}(\Gamma)$,

$$
\frac{\mathbf{e}(\mathcal{V})}{\langle\mathbf{a}\rangle \mathbf{x}^{l}}=\mathbf{e}\left(\mathcal{V}^{\prime}\right)
$$

with $\mathcal{V}^{\prime}=\mathcal{V}_{\left|\Gamma_{e}\right|}^{\prime}$ as in (44.7). Thus, in this case, by [27, Section 2.2]

$$
\begin{aligned}
\sum_{\Gamma_{e}} Q^{\left|\Gamma_{e}\right|}\left(\frac{\alpha_{\mu_{v}(e)}-\alpha_{\mu(v)}}{\mathfrak{d}(e)}\right)^{-b_{e}-1} \int_{\mathcal{Z}_{\Gamma_{e}}} \frac{\mathbf{e}(\mathcal{V}) \mathrm{ev}_{e}^{*} \phi_{\mu(v)}}{\langle\mathbf{a}\rangle \alpha_{\mu(v)}^{l} \mathbf{e}\left(\mathcal{N} \mathcal{Z}_{\Gamma_{e}}\right)} \prod_{s \in S_{e}}\left(\frac{\mathrm{ev}_{s}^{*} \phi_{i_{s}}}{\hbar_{s}-\psi_{s}}\right) \\
\quad=\sum_{\Gamma_{e}} Q^{\left|\Gamma_{e}\right|}\left(\frac{\alpha_{\mu_{v}(e)}-\alpha_{\mu(v)}}{\mathfrak{d}(e)}\right)^{-b_{e}-1} \int_{\mathcal{Z}_{\Gamma_{e}}} \frac{\mathbf{e}\left(\mathcal{V}^{\prime}\right) \mathrm{ev}_{e}^{*} \phi_{\mu(v)}}{\mathbf{e}\left(\mathcal{N} \mathcal{Z}_{\Gamma_{e}}\right)}=-\underset{\hbar_{e}=0}{\Re}\left\{\hbar_{e}^{-b_{e}} \widetilde{\mathcal{Z}}^{*}\left(\hbar_{e}, \alpha_{\mu(v)}, Q\right)\right\},
\end{aligned}
$$

where the sum is taken over all possibilities for the strand $\Gamma_{e}$, leaving the vertex $v$, with $\mu(v)$ fixed. By a similar reasoning, if $e \in \mathrm{E}_{v}^{*}(\Gamma)$,

$$
\begin{aligned}
& \sum_{\Gamma_{e}} Q^{\left|\Gamma_{e}\right|}\left(\frac{\alpha_{\mu_{v}(e)}-\alpha_{\mu(v)}}{\mathfrak{d}(e)}\right)^{-b_{e}-1} \int_{\mathcal{Z}_{\Gamma_{e}}} \frac{\mathbf{e}(\mathcal{V}) \mathrm{ev}_{e}^{*} \phi_{\mu(v)}}{\langle\mathbf{a}\rangle \alpha_{\mu(v)}^{l} \mathbf{e}\left(\mathcal{N} \mathcal{Z}_{\Gamma_{e}}\right)} \prod_{s \in S_{e}}\left(\frac{\mathrm{ev}_{s}^{*} \phi_{i_{s}}}{\hbar_{s}-\psi_{s}}\right)
\end{aligned}
$$

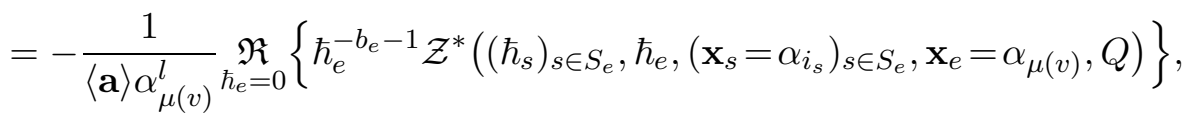

where the sum is taken over all possibilities for the strand $\Gamma_{e}$, leaving the vertex $v$, with $\mu(v)$ fixed, $\left|\Gamma_{e}\right|>0$, and carrying the marked points $S_{e} \subset[N]$, and $\mathcal{Z}^{*}$ is the positive-degree part of the power series (4.4) with $[N]$ replaced by $S_{e} \sqcup\{e\}$ if $\left|S_{e}\right| \geq 2$ (for $\left|S_{e}\right|=1, \mathcal{Z}^{*}$ is defined in (4.10) 13 Finally, if $s \in \eta^{-1}(v)$,

$$
\hbar_{s}^{-b_{s}-1} \prod_{k \neq i_{s}}\left(\alpha_{\mu(v)}-\alpha_{k}\right)=\frac{(-1)^{b_{s}}}{\langle\mathbf{a}\rangle \alpha_{\mu(v)}^{l}} \underset{\hbar_{e}=0}{\Re}\left\{\hbar_{e}^{-b_{s}-1} \llbracket \mathcal{Z}\left(\hbar_{s}, \hbar_{e}, \alpha_{i_{s}}, \alpha_{\mu(v)}, Q\right) \rrbracket_{Q ; 0}\right\} .
$$

This corresponds to the strand $\Gamma_{e}$ in (4.28) with $\left|\Gamma_{e}\right|=0$ whenever $S_{e}=\{s\}$ is a single-element set. On the other hand, if $\left|S_{e}\right| \geq 2$,

$$
\underset{\hbar_{e}=0}{\mathfrak{R}}\left\{\hbar_{e}^{-b_{e}-1} \llbracket \mathcal{Z}\left(\left(\hbar_{s}\right)_{s \in S_{e}}, \hbar_{e},\left(\mathbf{x}_{s}=\alpha_{i_{s}}\right)_{s \in S_{e}}, \mathbf{x}_{e}=\alpha_{\mu(v)}, Q\right) \rrbracket_{Q ; 0}\right\}=0
$$

Putting this all together, taking into account the group of symmetries (permutations of the onemarked strands), and summing over all possibilities for $m^{\prime} \equiv\left|\mathrm{E}_{v}^{\prime}(\Gamma)\right|$, while keeping

$$
m \equiv\left|\mathrm{E}_{v}^{*}(\Gamma)\right| \geq 3, \quad\left\{S_{i}\right\}_{i \in[m]} \equiv\left\{S_{e}\right\}_{e \in \mathrm{E}_{v}^{*}(\Gamma)} \in \mathcal{P}_{m}([N]), \quad \text { and } \quad j \equiv \eta(v) \in[n]
$$

\footnotetext{
${ }^{13}$ By the proof of [15. Chapter 30, (3.21)], LHS of (4.27) summed over $\Gamma_{e}$ with $\mathfrak{d}(e)=d$ and $\mu_{v}(e)=i$ fixed is the residue of $\hbar^{-b} \widetilde{\mathcal{Z}}^{*}\left(\hbar, \alpha_{\mu(v)}, Q\right)$ at $\hbar=\left(\alpha_{i}-\alpha_{\mu(v)}\right) / d$; see also [27, Section 2.2]. Since $\widetilde{\mathcal{Z}}^{*}\left(\hbar, \alpha_{\mu(v)}, Q\right)$ vanishes to second order at $\hbar=\infty, \hbar^{-b} \widetilde{\mathcal{Z}}^{*}\left(\hbar, \alpha_{\mu(v)}, Q\right) \mathrm{d} \hbar$ has no residue at $\hbar=\infty$ for all $b \in \overline{\mathbb{Z}}^{+}$. Since $\widetilde{\mathcal{Z}}^{*}\left(\hbar, \alpha_{\mu(v)}, Q\right) \mathrm{d} \hbar$ has poles only at $\hbar=\left(\alpha_{i}-\alpha_{\mu(v)}\right) / d$ with $i \in[n]-\mu(v)$ and $d \in \mathbb{Z}^{+}$, and at $\hbar=0$, 4.27) follows from the Residue Theorem on $S^{2}$. By (4.22), the same reasoning applies to $\hbar^{-1} \mathcal{Z}^{*}\left(\hbar_{s}, \hbar, \alpha_{i_{s}}, \alpha_{\mu(v)}, Q\right)$, giving the $\left|S_{e}\right|=1$ case of (4.28). Since $\left|\mathrm{E}_{v}^{*}(\Gamma)\right| \geq 3$, $\left|S_{e} \sqcup\{e\}\right|<N$; by Theorem $\mathrm{B}$ and induction on $N$, the same reasoning is applicable to (4.28) for $\left|S_{e}\right| \geq 2$ as well.
} 
fixed, we find that

$$
\begin{aligned}
& \frac{\prod_{k \neq j}\left(\alpha_{j}-\alpha_{k}\right)}{\langle\mathbf{a}\rangle \alpha_{j}^{l}} \sum_{\Gamma} Q^{|\Gamma|} \int_{\mathcal{Z}_{\Gamma}} \frac{\mathbf{e}(\mathcal{V})}{\mathbf{e}\left(\mathcal{N} \mathcal{Z}_{\Gamma}\right)} \prod_{s=1}^{s=N}\left(\frac{\mathrm{ev}_{s}^{*} \phi_{i_{s}}}{\hbar_{s}-\psi_{s}}\right)=\sum_{\mathbf{b} \in\left(\overline{\mathbb{Z}}^{+}\right)^{m}}\left\{\widetilde{\mathcal{Z}}_{m-3,\|\mathbf{b}\|}\left(\alpha_{j}, Q\right)\right. \\
& \left.\times \prod_{i=1}^{i=m}\left(\frac{1}{\langle\mathbf{a}\rangle \alpha_{j}^{l}} \frac{(-1)^{b_{i}}}{b_{i} !} \underset{\hbar_{i}^{\prime}=0}{\mathfrak{R}}\left\{\hbar_{i}^{\prime-b_{i}-1} \mathcal{Z}\left(\left(\hbar_{s}\right)_{s \in S_{i}}, \hbar_{i}^{\prime},\left(\alpha_{i_{s}}\right)_{s \in S_{i}}, \alpha_{j}, Q\right)\right\}\right)\right\} .
\end{aligned}
$$

By (4.17) and the first statement of Lemma B.1, the right-hand side of this expression reduces to

$$
\begin{aligned}
& \sum_{\mathbf{b} \in\left(\overline{\mathbb{Z}}^{+}\right)^{m}} \sum_{\substack{\mathbf{b}^{\prime \prime} \in\left(\overline{\mathbb{Z}}^{+}\right)^{m} \\
\mathbf{c} \in\left(\overline{\mathbb{Z}}^{+}\right)^{\infty} \\
\mathbf{b}^{\prime \prime} \mid+\|\mathbf{c}\|=m-3}}\left\{\Psi_{m-3, \mathbf{c}}\left(\alpha_{j}, Q\right)\right. \\
& \left.\times \prod_{i=1}^{i=m}\left(\frac{1}{\langle\mathbf{a}\rangle \alpha_{j}^{l}} \frac{1}{b_{i} !}\left(\begin{array}{c}
b_{i} \\
b_{i}^{\prime \prime}
\end{array}\right) \underset{\hbar_{i}^{\prime}=0}{\Re}\left\{\hbar_{i}^{\prime-b_{i}^{\prime \prime}-1}\left(-\frac{\zeta\left(\alpha_{j}, Q\right)}{\hbar_{i}^{\prime}}\right)^{b_{i}-b_{i}^{\prime \prime}} \mathcal{Z}\left(\left(\hbar_{s}\right)_{s \in S_{i}}, \hbar_{i}^{\prime},\left(\alpha_{i_{s}}\right)_{s \in S_{i}}, \alpha_{j}, Q\right)\right\}\right)\right\} \\
& =\sum_{\substack{\mathbf{b}^{\prime \prime} \in\left(\overline{\mathbb{Z}}^{+}\right)^{m} \\
\mathbf{c} \in\left(\overline{\mathbb{Z}}^{+}\right)^{\infty} \\
\left|\mathbf{b}^{\prime \prime}\right|+\|\mathbf{c}\|=m-3}}\left\{\Psi_{m-3, \mathbf{c}}\left(\alpha_{j}, Q\right) \prod_{i=1}^{i=m}\left(\frac{1}{\langle\mathbf{a}\rangle \alpha_{j}^{l}} \frac{1}{b_{i}^{\prime \prime !} !} \underset{\hbar=0}{\Re}\left\{\frac{e^{-\frac{\zeta\left(\alpha_{j}, Q\right)}{\hbar}}}{\hbar^{b_{i}^{\prime \prime}+1}} \mathcal{Z}\left(\left(\hbar_{s}\right)_{s \in S_{i}}, \hbar,\left(\alpha_{i_{s}}\right)_{s \in S_{i}}, \alpha_{j}, Q\right)\right\}\right)\right\} .
\end{aligned}
$$

Since $m \geq 3,\left|S_{i}\right| \leq N-2$ for every $i \in[m]$. Thus, each of the power series $\mathcal{Z}^{*}$ appearing in the last expression above is described either by (4.22) or Theorem $\mathrm{B}$ with $N$ replaced by $\left|S_{i}\right|+1<N$ (which we can assume to hold by induction). By the last expression for the left-hand side of (4.30), Lemma 4.4, (3.5) with $N$ replaced by $\left|S_{i}\right|+1<N$ whenever $\left|S_{i}\right| \geq 2$, and (4.12), the sum on the left hand-side side of (4.30) equals

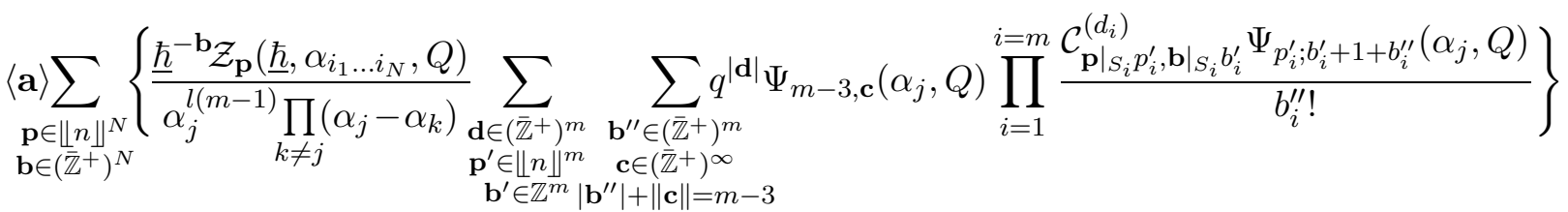

with $\Psi_{p ; b} \equiv 0$ if $b<0$. In the two-pointed case (for $\left|S_{i}\right|=1$ ), the above structure constants are given by

$$
\sum_{d=0}^{\infty} q^{d} \mathcal{C}_{p p^{\prime}, b b^{\prime}}^{(d)}=\delta_{b+b^{\prime},-1}(-1)^{b} \mathcal{C}_{p p^{\prime}}(Q)
$$

with $\mathcal{C}_{p p^{\prime}}$ as in (4.23). Summing over all

$$
j \in[n], \quad \mathbf{S} \equiv\left\{S_{i}\right\}_{\in[m]} \in \mathcal{P}_{m}([N]), \quad \text { and } \quad m \geq 3
$$


and using the Residue Theorem on $S^{2}$, we obtain a recursion for the coefficients $\mathcal{C}_{\mathbf{p}, \mathbf{b}}^{(d)}$ in Theorem B:

$$
\begin{aligned}
& \mathcal{C}_{\mathbf{p}, \mathbf{b}}^{(d)}=-\sum_{\substack{m, d^{\prime} \in \overline{\mathbb{Z}}^{+} \\
m \geq 3}} \sum_{\substack{\mathbf{S} \in \mathcal{P}_{m}([N]) \\
\mathbf{d} \in \mathcal{P}_{m}\left(d-d^{\prime}\right) \\
\left(\mathbf{p}^{\prime}, \mathbf{b}^{\prime}\right) \in\left\lfloor n \rrbracket^{m} \times \mathbb{Z}^{m}\right.}} \sum_{\begin{array}{r}
\mathbf{b}^{\prime \prime} \in\left(\overline{\mathbb{Z}}^{+}\right)^{m} \\
\mathbf{c} \in\left(\overline{\mathbb{Z}}^{+}\right)^{\prime} \\
\left|\mathbf{b}^{\prime \prime}\right|+\|\mathbf{c}\|=m-3
\end{array}} \\
& \underset{\mathbf{x}=0, \infty}{\mathfrak{R}} \llbracket \frac{\Psi_{m-3, \mathbf{c}}(\mathbf{x}, Q)}{\mathbf{x}^{l(m-1)} \prod_{k=1}^{k=n}\left(\mathbf{x}-\alpha_{k}\right)} \prod_{i=1}^{i=m} \frac{\mathcal{C}_{\mathbf{p}\left|S_{i} p_{i}^{\prime}, \mathbf{b}\right|_{S_{i}} b_{i}^{\prime}}^{\left(d_{i}\right)} \Psi_{p_{i}^{\prime} ; b_{i}^{\prime}+1+b_{i}^{\prime \prime}}(\mathbf{x}, Q)}{b_{i}^{\prime \prime} !} \|_{q ; d^{\prime}} .
\end{aligned}
$$

By (3.5) and (3.4) , if $\mathbf{b} \in\left(\overline{\mathbb{Z}}^{+}\right)^{N}$ and $d \in \overline{\mathbb{Z}}^{+}$, the coefficient of

$$
q^{d} \prod_{s=1}^{s=N}\left(\left(\hbar_{s}^{-1}\right)^{b_{s}+1}\right)
$$

in the power series $\mathcal{Z}(\underline{\hbar}, \underline{\mathbf{x}}, Q)$ is

$$
\begin{aligned}
\llbracket \mathcal{Z}(\underline{\hbar}, \underline{\mathbf{x}}, Q) \rrbracket_{\underline{\hbar}^{-1}, q ; \mathbf{b}+\mathbf{1}, d}= & \sum_{\mathbf{p} \in \llbracket n \rrbracket^{N}} \mathcal{C}_{\mathbf{p}, \mathbf{b}}^{(d)} \underline{\mathbf{x}}_{s}^{\mathbf{p}} \\
& +\sum_{\substack{d^{\prime} \in\left\lfloor d \rrbracket \\
\mathbf{d} \in \mathcal{P}_{N}\left(d-d^{\prime}\right)\right.}} \sum_{\mathbf{p} \in \llbracket n \rrbracket^{N}} \sum_{\substack{\mathbf{b}^{\prime} \in\left(\overline{\mathbb{Z}}^{+}\right)^{N} \\
b_{s}^{\prime} \leq b_{s}}} \mathcal{C}_{\mathbf{p}, \mathbf{b}^{\prime}}^{\left(d^{\prime}\right)} \prod_{s=1}^{s=N} \llbracket \mathcal{Z}_{\left(p_{s}\right)}\left(\hbar_{s}, \mathbf{x}_{s}, Q\right) \rrbracket_{\hbar_{s}^{-1}, q ; b_{s}-b_{s}^{\prime}, d_{s}},
\end{aligned}
$$

where $\llbracket \mathcal{Z}_{(p)}(\hbar, \mathbf{x}, Q) \rrbracket_{\hbar^{-1}, q ; b, d^{\prime}}$ is the coefficient of $q^{d^{\prime}}\left(\hbar^{-1}\right)^{b}$ in

$$
\mathcal{Z}_{(p)}(\hbar, \mathbf{x}, Q) \equiv \frac{\mathcal{Z}_{p}(\hbar, \mathbf{x}, Q)}{\prod_{r=p_{s}-l+1}^{n-l-1} I_{r}\left(q_{s}\right)} \in H_{\mathbb{T}}^{*}\left(\mathbb{P}^{n-1}\right)\left[\left[\hbar^{-1}, Q\right]\right]=H_{\mathbb{T}}^{*}\left(\mathbb{P}^{n-1}\right)\left[\left[\hbar^{-1}, Q\right]\right] .
$$

Since $H_{\mathbb{T}}^{*}\left(\mathbb{P}^{n-1}\right)$ and $H_{\mathbb{T}}^{*}\left(\mathbb{P}_{N}^{n-1}\right)$ are free modules over $\mathbb{Q}[\alpha]$ with bases $\left\{\mathbf{x}^{p}\right\}_{p \in\lfloor n \rrbracket}$ and $\left\{\underline{\mathbf{x}}^{\mathbf{p}}\right\}_{\mathbf{p} \in \llbracket n \rrbracket^{N}}$, respectively, and

$$
\llbracket \mathcal{Z}_{(p)}(\hbar, \mathbf{x}, Q) \rrbracket_{\hbar^{-1}, q ; b, d^{\prime}} \in H_{\mathbb{T}}^{*}\left(\mathbb{P}^{n-1}\right), \quad \llbracket \mathcal{Z}(\underline{\hbar}, \underline{\mathbf{x}}, Q) \rrbracket_{\underline{\hbar}^{-1}, q ; \mathbf{b}+\mathbf{1}, d} \in H_{\mathbb{T}}^{*}\left(\mathbb{P}_{N}^{n-1}\right)
$$

by (3.4) and (3.3), (4.33) and induction on $d$ imply that $\mathcal{C}_{\mathbf{p}, \mathbf{b}}^{(d)} \in \mathbb{Q}[\alpha]$ as claimed in Theorem $\mathrm{B}$,

We now confirm (3.6) by induction on $N$. For $N=2$, (3.6) holds by (4.31), (4.21), and (2.31). On the other hand, by (4.32), (4.18), the second statement in (4.13), the inductive assumption (3.6), and the last two statements in Lemma 4.1.

$$
\begin{aligned}
& \mathcal{C}_{\mathbf{p}, \mathbf{b}}^{(d)} \sim-\sum_{\substack{m, d^{\prime} \in \overline{\mathbb{Z}}^{+} \\
m \geq 3}} \sum_{\substack{\mathbf{S} \in \mathcal{P}_{m}([N]) \\
\mathbf{d} \in \mathcal{P}_{m}\left(d-d^{\prime}\right) \\
\mathbf{t} \in\left(\overline{\mathbb{Z}}^{+}\right)^{m} \\
\left(\mathbf{p}^{\prime}, \mathbf{b}^{\prime}\right) \in\left\lfloor n \rrbracket^{m} \times \mathbb{Z}^{m}\right.}} \sum_{\substack{\mathbf{b}^{\prime \prime} \in\left(\overline{\mathbf{Z}}^{+}\right)^{\prime \prime} \in\left(+\|\mathbf{C}\|=m-3 \\
\mathbf{c}\left(\mathbb{Z}^{+}\right)\right.}} \hat{\sigma}_{n}^{|\mathbf{t}|} \\
& \underset{\mathbf{x}=0, \infty}{\mathfrak{R}}\left\|\frac{\mathbf{x}^{\left|\mathbf{p}^{\prime}\right|-\left|\mathbf{b}^{\prime}\right|-(l+2)(m-1)+1}}{\prod_{k=1}^{k=n}\left(\mathbf{x}-\alpha_{k}\right)} \Phi_{m-3, \mathbf{c}}(\mathbf{q}) \prod_{i=1}^{i=m}\left(\mathbf{c}_{\mathbf{p}\left|{ }_{S_{i}} p_{i}^{\prime}, \mathbf{b}\right|_{S_{i}} b_{i}^{\prime}} \frac{I_{0}(\mathbf{q})^{2} \Phi_{p_{i}^{\prime} ; b_{i}^{\prime}+1+b_{i}^{\prime \prime}}(\mathbf{q})}{b_{i}^{\prime \prime} ! L(\mathbf{q})^{\delta_{0 \nu_{\mathbf{a}}} n} \Phi_{0}(\mathbf{q})}\right)\right\|_{q ; d^{\prime}}
\end{aligned}
$$


Since $\mathbf{q}=q / \mathbf{x}^{\nu_{\mathbf{a}}}$, by the last statement of Lemma 4.1 the negative of the expression on the last line is equivalent to

$$
\llbracket \Phi_{m-3, \mathbf{c}}(q) \prod_{i=1}^{i=m}\left(\mathrm{c}_{\left.\mathbf{p}\right|_{S_{i}} p_{i}^{\prime},\left.\mathbf{b}\right|_{S_{i}} b_{i}^{\prime}} \frac{I_{0}(q)^{2} \Phi_{p_{i}^{\prime} ; b_{i}^{\prime}+1+b_{i}^{\prime \prime}}(q)}{b_{i}^{\prime \prime} ! L(q)^{\delta_{0 \nu_{\mathbf{a}}} n} \Phi_{0}(q)}\right) \|_{q ; d^{\prime}} \hat{\sigma}_{n}^{t^{\prime}}
$$

with $t^{\prime} \in \mathbb{Z}$ defined by

$$
\left|\mathbf{p}^{\prime}\right|-\left|\mathbf{b}^{\prime}\right|-(l+2)(m-1)+1-\nu_{\mathbf{a}} d^{\prime}=n-1+n t^{\prime} \quad \Longleftrightarrow \quad\left(\mathbf{p}^{\prime}, \mathbf{b}^{\prime}\right) \in \mathcal{S}_{m}\left(d^{\prime}, t^{\prime}\right) ;
$$

if such an integer $t^{\prime}$ does not exist, the above residue is equivalent to 0 . Since $\mathcal{C}_{\mathbf{p}, \mathbf{b}}^{(d)} \in \mathbb{Q}[\alpha]$ by the previous paragraph, we conclude that

$$
\begin{aligned}
& \mathcal{C}_{\mathbf{p}, \mathbf{b}}^{(d)} \sim \sum_{t=0}^{\infty} \hat{\sigma}_{n}^{t} \sum_{\substack{m, d^{\prime}, t^{\prime} \in \mathbb{Z} \\
m \geq 3}} \sum_{\substack{\mathbf{S} \in \mathcal{P}_{m}([N]) \\
\mathbf{d} \in \mathcal{P}_{m}\left(d-d^{\prime}\right) \\
\mathbf{t} \in \mathcal{P}_{m}\left(t-t^{\prime}\right) \\
\left(\mathbf{p}^{\prime}, \mathbf{b}^{\prime}\right) \in \mathcal{S}_{m}\left(d^{\prime}, t^{\prime}\right)}} \sum_{\substack{\left|\mathbf{b}^{\prime \prime}\right|+\|\mathbf{c}\|=m-3 \\
\mathbf{c} \in\left(\mathbb{Z}^{+}\right)^{\infty}}}\left(\left(\prod_{i=1}^{i=m} \mathrm{c}_{\left.\mathbf{p}\right|_{S_{i}} p_{i}^{\prime},\left.\mathbf{b}\right|_{S_{i}} b_{i}^{\prime}}^{\left(d_{i}, t_{i}\right)}\right)\right. \\
& \left.\times \llbracket \Phi_{m-3, \mathbf{c}}(q) \prod_{i=1}^{i=m} \frac{I_{0}(q)^{2} \Phi_{p_{i}^{\prime} ; b_{i}^{\prime}+1+b_{i}^{\prime \prime}}(q)}{b_{i}^{\prime \prime} ! L(q)^{\delta_{0 \nu \mathbf{a}}} \Phi_{0}(q)} \|_{q ; d^{\prime}}\right) .
\end{aligned}
$$

Comparing this expression with (2.32), we conclude that (3.6) holds 14

We next show that (3.6) holds with the coefficients $\mathrm{c}_{\mathbf{p}, \mathbf{b}}^{(d, t)}$ as defined in (2.47). Let $\Gamma$ be an $N$-marked decorated tree and $\bar{\Gamma}$ its core as before, with a partial ordering $\prec$ as in Section 2.4. This time, we break $\Gamma$ and $\overline{\mathcal{Z}}_{\Gamma}$ at all vertices $\overline{\operatorname{Ver}} \subset$ Ver of $\bar{\Gamma}$, adding a marked point to each of the strands; see Figure 3, There are now three types of strands, (S1)-(S3), described in Section 4.1. Each strand of type (S3) carries one of the original marked points $s \in[N]$ and an added marked point $s^{\prime}$, which we associate with the element of $\mathrm{E}_{v}(\Gamma)$ that leaves $v$ in the direction of $\eta(s)$. These strands are thus naturally indexed by the complement of the subset $\eta^{-1}(\overline{\mathrm{Ver}}) \subset[N]$ of the marked points attached to a vertex of the core in $\Gamma$. Each strand of type (S2) runs between vertices in $\overline{\operatorname{Ver}} \subset$ Ver in $\Gamma$ that are joined by an edge $e=\left\{v_{e}^{-}, v_{e}^{+}\right\}$in $\bar{\Gamma}$, with $v_{e}^{-} \prec v_{e}^{+}$. It carries two added marked points, which we label $e^{-}$and $e^{+}$, attached to the vertices $v_{e}^{-}$and $v_{e}^{+}$, respectively, in the strand $\Gamma_{e}$. We associate the marked point $e^{-}\left(\operatorname{resp} . e^{+}\right)$with the element of $\mathrm{E}_{v_{e}^{-}}(\Gamma)\left(\operatorname{resp} . \mathrm{E}_{v_{e}^{+}}(\Gamma)\right)$ that leaves $v_{e}^{-}$ (resp. $v_{e}^{+}$) in the directions of $v_{e}^{+}$(resp. $v_{e}^{-}$). Similarly to the first approach, for each $v \in \overline{\text { Ver}}$, denote by $\mathrm{E}_{v}^{\prime}(\Gamma) \subset \mathrm{E}_{v}(\Gamma)$ the set of one-marked edges at $v$ and set

$$
\overline{\mathrm{E}}_{v}(\Gamma)=\mathrm{E}_{v}^{\prime}(\Gamma) \cup \eta^{-1}(v) \cup \mathrm{E}_{v}(\bar{\Gamma}) \subset \mathrm{E}_{v}(\Gamma) \sqcup[N], \quad \overline{\mathrm{E}}(\Gamma)=\bigsqcup_{v \in \overline{\mathrm{Ver}}} \overline{\mathrm{E}}_{v}(\Gamma) .
$$

As before, this set indexes the marked points on the contracted component.

\footnotetext{
${ }^{14}$ As can be seen by induction on $n, \mathcal{I} \mathbb{Q}_{\alpha}^{\prime} \cap \mathbb{Q}[\alpha]=\mathcal{I}$. Since $\mathcal{C}_{\mathbf{p}, \mathbf{b}}^{(d)}$ is a symmetric function in $\alpha_{1}, \ldots, \alpha_{n}$, it is even sufficient to check that the symmetric polynomials in $\mathcal{I} \mathbb{Q}_{\alpha}^{\prime} \cap \mathbb{Q}[\alpha]$ are contained in $\mathcal{I}$; this is immediate from the algebraic independence of the elementary symmetric functions.
} 
The analogues of the decompositions (4.24) in this case are

$$
\begin{gathered}
\mathcal{Z}_{\Gamma}=\prod_{v \in \overline{\operatorname{Ver}}}\left(\overline{\mathcal{M}}_{0, \overline{\mathrm{E}}_{v}(\Gamma)} \times \prod_{e \in \mathrm{E}_{v}^{\prime}(\Gamma)} \mathcal{Z}_{\Gamma_{e}}\right) \times \prod_{e \in \overline{\mathrm{Edg}}} \mathcal{Z}_{\Gamma_{e}}, \\
\frac{\mathbf{e}(\mathcal{V})}{\prod_{v \in \overline{\operatorname{Ver}}} \mathbf{e}\left(\mathcal{L}_{\bar{\mu}(v)}\right)}=\prod_{v \in \overline{\operatorname{Ver}}} \prod_{e \in \mathrm{E}_{v}^{\prime}(\Gamma)} \frac{\pi_{e}^{*} \mathbf{e}(\mathcal{V})}{\mathbf{e}\left(\mathcal{L}_{\bar{\mu}(v)}\right)} \times \prod_{e \in \overline{\operatorname{Edg}}} \frac{\pi_{e}^{*} \mathbf{e}(\mathcal{V})}{\mathbf{e}\left(\mathcal{L}_{\bar{\mu}\left(v_{e}^{-}\right)}\right) \mathbf{e}\left(\mathcal{L}_{\bar{\mu}\left(v_{e}^{+}\right)}\right)}, \\
\frac{\prod_{v \in \overline{\operatorname{Ver}}} \mathbf{e}\left(T_{\bar{\mu}(v)} \mathbb{P}^{n-1}\right)}{\mathbf{e}\left(\mathcal{N Z}_{\Gamma}\right)}=\prod_{v \in \overline{\operatorname{Ver}}}\left(\prod_{e \in \mathrm{E}_{v}(\Gamma)} \frac{\mathbf{e}\left(T_{\bar{\mu}(v)} \mathbb{P}^{n-1}\right)}{\hbar_{e}^{\prime}-\pi_{e}^{*} \psi_{e}} \times \prod_{e \in \mathrm{E}_{v}^{\prime}(\Gamma)} \frac{1}{\mathbf{e}\left(\mathcal{N} \mathcal{Z}_{\Gamma_{e}}\right)}\right) \times \prod_{e \in \overline{\operatorname{Edg}}} \frac{1}{\mathbf{e}\left(\mathcal{N}_{\mathcal{Z}_{e}}\right)},
\end{gathered}
$$

For each $v \in \overline{\mathrm{Ver}}$, (4.25) still applies. The analogue of (4.26), but weighted by the automorphism group, is then

$$
\begin{aligned}
& \left(\prod_{v \in \overline{\mathrm{Ver}}} \frac{\prod_{k \neq \bar{\mu}(v)}\left(\alpha_{\bar{\mu}(v)}-\alpha_{k}\right)}{\langle\mathbf{a}\rangle \alpha_{\bar{\mu}(v)}^{l}}\right) \int_{\mathcal{Z}_{\Gamma}} \frac{\mathbf{e}(\mathcal{V})}{\mathbf{e}\left(\mathcal{N} \mathcal{Z}_{\Gamma}\right)} \prod_{s=1}^{s=N}\left(\frac{\mathrm{ev}_{s}^{*} \phi_{i_{s}}}{\hbar_{s}-\psi_{s}}\right) \\
& =\sum_{\mathbf{b} \in\left(\overline{\mathbb{Z}}^{+}\right)_{\overline{\mathrm{E}}(\Gamma)}}\left\{\prod _ { v \in \overline { \operatorname { V e r } } } \left(\frac{\left(\left|\overline{\mathrm{E}}_{v}(\Gamma)\right|-3\right) !}{\left|\mathrm{E}_{v}^{\prime}(\Gamma)\right| !} \prod_{e \in \mathrm{E}_{v}^{\prime}(\Gamma)}\left(\frac{1}{b_{e} !}\left(\frac{\alpha_{\bar{\mu}(v)}-\alpha_{\mu_{v}(e)}}{\mathfrak{d}(e)}\right)^{-b_{e}-1} \int_{\mathcal{Z}_{\Gamma_{e}}} \frac{\mathbf{e}(\mathcal{V}) \mathrm{ev}_{e}^{*} \phi_{\bar{\mu}(v)}}{\langle\mathbf{a}\rangle \alpha_{\bar{\mu}(v)}^{l} \mathbf{e}\left(\mathcal{N} \mathcal{Z}_{\Gamma_{e}}\right)}\right)\right.\right. \\
& |\mathbf{b}|_{\overline{\mathrm{E}}_{v}(\Gamma)}=\left|\overline{\mathrm{E}}_{v}(\Gamma)\right|-3 \\
& \left.\times \prod_{s \in \bar{\eta}^{-1}(v)}\left(\frac{1}{b_{s^{\prime}} !}\left(\frac{\alpha_{\bar{\mu}(v)}-\alpha_{\mu_{v}\left(s^{\prime}\right)}}{\mathfrak{d}\left(s^{\prime}\right)}\right)^{-b_{s^{\prime}}-1} \int_{\mathcal{Z}_{\Gamma_{s^{\prime}}}} \frac{\mathbf{e}(\mathcal{V}) \mathrm{ev}_{s^{\prime}}^{*} \phi_{\bar{\mu}(v)} \mathrm{ev}_{s}^{*} \phi_{i_{s}}}{\langle\mathbf{a}\rangle \alpha_{\bar{\mu}(v)}^{l} \mathbf{e}\left(\mathcal{N} \mathcal{Z}_{\Gamma_{s^{\prime}}}\right)\left(\hbar_{s}-\psi_{s}\right)}\right)\right) \\
& \left.\times \prod_{e \in \overline{\mathrm{Edg}}}\left(\frac{1}{b_{e^{-}} ! b_{e^{+}} !} \prod_{*=-,+}\left(\frac{\alpha_{\bar{\mu}\left(v_{e}^{*}\right)}-\alpha_{\mu_{v_{e}^{*}}\left(e^{*}\right)}}{\mathfrak{d}\left(e^{*}\right)}\right)^{-b_{e^{*-}}} \times \int_{\mathcal{Z}_{\Gamma_{e}}} \frac{\left.\mathbf{e}(\mathcal{V}) \mathrm{ev}_{e^{-}}^{*} \phi_{\bar{\mu}\left(v_{e}^{-}\right)} \mathrm{ev}_{e^{+}}^{*} \phi_{\bar{\mu}\left(v_{e}^{+}\right)}\right)}{\left\langle\mathbf{a} \alpha_{\bar{\mu}\left(v_{e}^{-}\right)}^{l} \alpha_{\bar{\mu}\left(v_{e}^{+}\right)}^{l} \mathbf{e}\left(\mathcal{N} \mathcal{Z}_{\Gamma_{e}}\right)\right.}\right)\right\}
\end{aligned}
$$

where

$$
\frac{1}{b_{s^{\prime}} !}\left(\frac{\alpha_{\bar{\mu}(v)}-\alpha_{\mu_{v}\left(s^{\prime}\right)}}{\mathfrak{d}\left(s^{\prime}\right)}\right)^{-b_{s^{\prime}}-1} \int_{\mathcal{Z}_{\Gamma_{s^{\prime}}}} \frac{\mathbf{e}(\mathcal{V}) \mathrm{ev}_{s^{\prime}}^{*} \phi_{\bar{\mu}(v)} \mathrm{ev}_{s}^{*} \phi_{i_{s}}}{\langle\mathbf{a}\rangle \alpha_{\bar{\mu}(v)}^{l} \mathbf{e}\left(\mathcal{N} \mathcal{Z}_{\Gamma_{s^{\prime}}}\right)\left(\hbar_{s}-\psi_{s}\right)} \equiv \frac{1}{b_{s} !}\left(\hbar_{s}^{-b_{s}-1} \prod_{k \neq i_{s}}\left(\alpha_{\bar{\mu}(v)}-\alpha_{k}\right)\right)
$$

if $s \in \eta^{-1}(v)$.

For each $v \in \overline{\mathrm{Ver}}$, (4.27) still reduces the summation of the factor on the second line in (4.34) over all possibilities for $\Gamma_{e}$ with $e \in \mathrm{E}_{v}^{\prime}(\Gamma)$ and for $m_{v}^{\prime} \equiv\left|\mathrm{E}_{v}^{\prime}(\Gamma)\right|$ to $\widetilde{\mathcal{Z}}_{m_{v},\left\|\mathbf{b}_{v}\right\|}\left(\alpha_{j_{v}}, Q\right)$, where

$$
m_{v} \equiv m_{v}(\bar{\Gamma})=\left|\bar{\eta}^{-1}(v)\right|+\left|\mathrm{E}_{v}(\bar{\Gamma})\right|-3, \quad \mathbf{b}_{v}=\left.\mathbf{b}\right|_{\bar{\eta}^{-1}(v) \cup \mathrm{E}_{v}(\bar{\Gamma})}, \quad j_{v}=\bar{\mu}(v) .
$$

For each $s \in \bar{\eta}^{-1}(v)$, (4.28) and (4.29) with $v=\bar{\eta}(s)$ and $S_{e}=\{s\}$ still compute the sum of the factors on the third line in (4.34) over all possibilities for $\Gamma_{s^{\prime}}$ of positive and zero degree, respectively. By a similar reasoning (see Footnote [13), for each $e \in \overline{\mathrm{Edg}}$

$$
\begin{aligned}
\sum_{\Gamma_{e}}\left(\left(\frac{\alpha_{\mu_{v_{e}^{-}}\left(e^{-}\right)}-\alpha_{j_{v_{e}^{-}}}}{\mathfrak{d}\left(e^{-}\right)}\right)^{-b_{e}^{-}-1}\left(\frac{\alpha_{\mu_{v_{e}^{+}}\left(e^{+}\right)}-\alpha_{j_{e}^{+}}}{\mathfrak{d}\left(e^{+}\right)}\right)^{-b_{e}^{+}-1} \int_{\mathcal{Z}_{\Gamma_{e}}} \frac{\mathbf{e}(\mathcal{V}) \mathrm{ev}_{e^{-}}^{*} \phi_{j_{v_{e}^{-}}} \mathrm{ev}_{e^{+}}^{*} \phi_{j_{v_{e}^{+}}}}{\mathbf{e}\left(\mathcal{N} \mathcal{Z}_{\Gamma_{e}}\right)}\right) \\
=\underset{\hbar_{-}=0}{\mathfrak{R}}\left\{\underset{\hbar_{+}=0}{\mathfrak{R}}\left\{\hbar_{-}^{-b_{e}^{-}-1} \hbar_{+}^{-b_{e}^{+}-1} \mathcal{Z}^{*}\left(\hbar_{-}, \hbar_{+}, \alpha_{j_{v_{e}^{-}}}, \alpha_{j_{v_{e}^{+}}}, Q\right)\right\}\right\},
\end{aligned}
$$


where the sum is taken over all possibilities for the strand $\Gamma_{e}$ between the vertices $v_{e^{-}}$and $v_{e^{+}}$in $\Gamma$ with $\mu\left(v_{e}^{-}\right)=j_{v_{e}^{-}}$and $\mu\left(v_{e}^{+}\right)=j_{v_{e}^{+}}$fixed. Since

$$
\underset{\hbar_{-}=0}{\Re}\left\{\underset{\hbar_{+}=0}{\mathfrak{R}}\left\{\hbar_{-}^{-b_{e}^{-}-1} \hbar_{+}^{-b_{e}^{+}-1} \frac{\langle\mathbf{a}\rangle \alpha_{j_{e}^{-}}^{l}}{\hbar_{-}+\hbar_{+}} \sum_{\begin{array}{c}
p_{-}+p_{+}+r=n-1 \\
p_{-}, p_{+}, r \geq 0
\end{array}} \hat{\sigma}_{r} \alpha_{v_{e}^{-}}^{p_{-}} \alpha_{j_{v_{e}^{+}}}^{p_{+}}\right\}\right\}=0 \quad \forall b_{e}^{-}, b_{e}^{+} \in \overline{\mathbb{Z}}^{+},
$$

we can replace $\mathcal{Z}^{*}$ in the previous expression by $\mathcal{Z}$.

Putting this all together, we obtain a replacement for (4.30), involving products over $v \in \overline{\text { Ver }}$ and $e \in \overline{\mathrm{Edg}}$, which (4.17) and the first statement of Lemma B.1, reduce to

$$
\begin{aligned}
& \left(\prod_{v \in \overline{\operatorname{Ver}}} \frac{\prod_{k \neq j_{v}}\left(\alpha_{j_{v}}-\alpha_{k}\right)}{\langle\mathbf{a}\rangle \alpha_{j_{v}}^{l}}\right) \sum_{\Gamma} Q^{|\Gamma|} \int_{\mathcal{Z}_{\Gamma}} \frac{\mathbf{e}(\mathcal{V})}{\mathbf{e}\left(\mathcal{N} \mathcal{Z}_{\Gamma}\right)} \prod_{s=1}^{s=N}\left(\frac{\mathrm{ev}_{s}^{*} \phi_{i_{s}}}{\hbar_{s}-\psi_{s}}\right) \\
& =\quad \sum_{\mathbf{b}^{\prime \prime} \in\left(\overline{\mathbb{Z}}^{+}\right)^{N}}\left\{\prod_{v \in \overline{\mathrm{Ver}}} \Psi_{m_{v}, \mathbf{c}_{v}}\left(\alpha_{j_{v}}, Q\right) \times \prod_{s=1}^{s=N}\left(\frac{1}{\langle\mathbf{a}\rangle \alpha_{j_{s}}^{l}} \frac{1}{b_{s}^{\prime \prime} !} \underset{\hbar=0}{\mathfrak{R}}\left\{\frac{e^{-\frac{\zeta\left(\alpha_{j_{s}}, Q\right)}{\hbar}}}{\hbar^{b_{s}^{\prime \prime}+1}} \mathcal{Z}\left(\hbar_{s}, \hbar, \alpha_{i_{s}}, \alpha_{j_{s}}, Q\right)\right\}\right)\right. \\
& \mathbf{b}^{-}, \mathbf{b}^{+} \in\left(\overline{\mathbb{Z}}^{+}\right)^{\overline{\mathrm{Edg}}} \\
& \left(\mathbf{c}_{v}\right)_{v \in \overline{\mathrm{Ver}}} \in\left(\left(\overline{\mathbb{Z}}^{+}\right)^{\infty}\right)^{\overline{\mathrm{Ver}}} \\
& \left|\mathbf{b}^{\prime \prime}\right|_{\eta^{-1}(v)}+\left|\mathbf{b}^{-}\right|_{\mathrm{E}_{\bar{\Gamma}}^{-}(v)}+b_{e_{v}}^{+}+\left\|\mathbf{c}_{v}\right\|=m_{v} \\
& \left.\times \prod_{e \in \overline{\operatorname{Edg}}}\left(\frac{1}{\langle\mathbf{a}\rangle^{2} \alpha_{j_{v_{e}^{-}}^{l}}^{l} \alpha_{j_{v_{e}^{+}}^{l}}^{l}} \frac{1}{b_{e}^{-} ! b_{e}^{+} !} \underset{\hbar_{-}}{\Re}\left\{\underset{\hbar_{+}=0}{\Re}\left\{\frac{e^{-\frac{\zeta\left(\alpha_{v_{e}}, Q\right)}{\hbar_{-}}-\frac{\zeta\left(\alpha_{j} v_{e}^{+}\right.}{\hbar_{+}}}}{\hbar_{-}^{b_{e}^{-}+1} \hbar_{+}^{b_{e}^{+}+1}} \mathcal{Z}\left(\hbar_{-}, \hbar_{+}, \alpha_{j_{v_{e}^{-}}}, \alpha_{j_{v_{e}^{+}}}, Q\right)\right\}\right\}\right)\right\},
\end{aligned}
$$

where $b_{e_{v_{0}}} \equiv 0$ for the minimal element $v_{0} \in \overline{\mathrm{Ver}}, j_{s}=j_{\bar{\mu}(\bar{\eta}(s))}$, and the sum is taken over all possibilities for $\Gamma$ with the core $\bar{\Gamma}=(\overline{\mathrm{Ver}}, \overline{\mathrm{Edg}} ; \bar{\mu}, \bar{\eta})$ fixed. Using Lemma 4.4 and (4.12) to compute the residues, we find that the sum on the left-hand side of the above expression equals

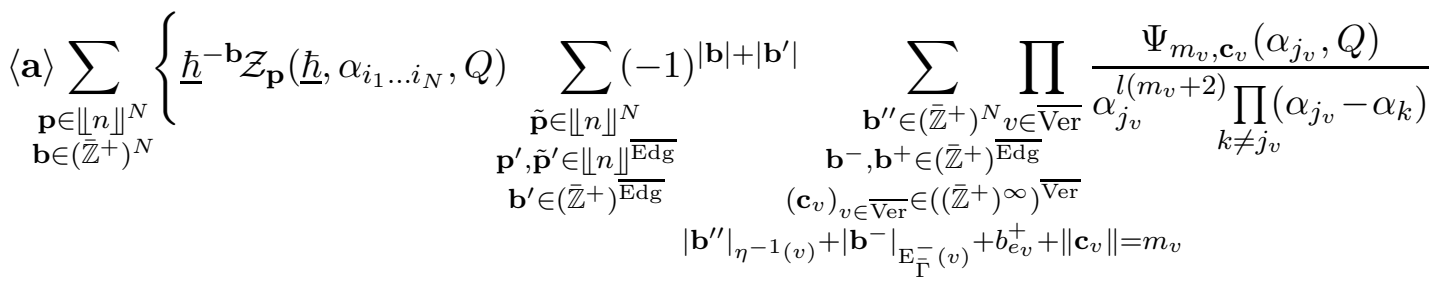

$$
\begin{aligned}
& \left.\times \prod_{s=1}^{s=N} \frac{\mathcal{C}_{p_{s} \tilde{p}_{s}}(Q) \Psi_{\tilde{p}_{s} ; b_{s}^{\prime \prime}-b_{s}}\left(\alpha_{j_{s}}, Q\right)}{b_{s}^{\prime \prime} !} \times \prod_{e \in \overline{\mathrm{Edg}}} \frac{\mathcal{C}_{p_{e}^{\prime} \tilde{p}_{e}}(Q) \Psi_{p_{e}^{\prime} ; b_{e}^{+}-b_{e}^{\prime}}\left(\alpha_{j_{v_{e}^{+}}}, Q\right) \Psi_{\tilde{p}_{e}^{\prime} ; b_{e}^{-}+1+b_{e}^{\prime}}\left(\alpha_{j_{v_{e}^{-}}}, Q\right)}{b_{e}^{-} ! b_{e}^{+} !}\right\}
\end{aligned}
$$

For each $v \in \overline{\mathrm{Ver}}$, we now sum up the product of the corresponding factors above over all possibilities for $j_{v} \in[n]$ (which also determines $j_{s}$ and $j_{v_{e}^{ \pm}}$whenever $\eta(s)=v$ and $v_{e}^{ \pm}=v$ ). Using the Residue 
Theorem on $S^{2}$, we now obtain an explicit formula for the coefficients $\mathcal{C}_{\mathbf{p}, \mathbf{b}}^{(d)}$ in Theorem $\mathbb{B}$;

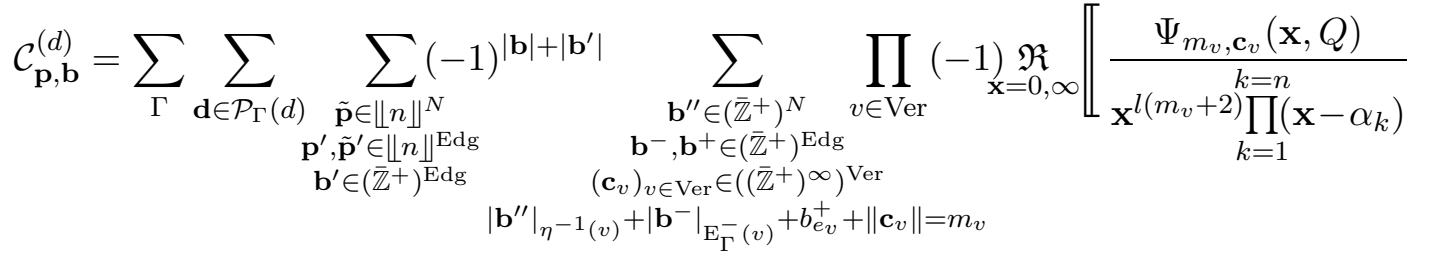

$$
\begin{aligned}
& \times \prod_{s \in \eta^{-1}(v)} \frac{\mathcal{C}_{p_{s} \tilde{p}_{s}}(Q) \Psi_{\tilde{p}_{s} ; b_{s}^{\prime \prime}-b_{s}}(\mathbf{x}, Q)}{b_{s}^{\prime \prime} !} \times \prod_{e \in \mathrm{E}_{\Gamma}^{-}(v)} \frac{\mathcal{C}_{p_{e}^{\prime} \tilde{p}_{e}^{\prime}}(Q) \Psi_{\tilde{p}_{e}^{\prime} ; b_{e}^{-}+1+b_{e}^{\prime}}(\mathbf{x}, Q)}{b_{e}^{-} !} \times \frac{\Psi_{p_{e_{v}}^{\prime} ; b_{e v}^{+}-b_{e_{v}}^{\prime}}(\mathbf{x}, Q)}{b_{e_{v}}^{+} !} \|_{q ; d_{v}}
\end{aligned}
$$

where the outer sum is taken over all $N$-marked trivalent trees $\Gamma \equiv($ Ver, Edg; $\eta)$ and

$$
\frac{\Psi_{p_{e_{v_{0}}}^{\prime} ; b_{e_{v_{0}}}^{+}-b_{e_{v_{0}}}^{\prime}}(\mathbf{x}, Q)}{b_{e_{v_{0}}}^{+} !} \equiv 1
$$

for the minimal element $v_{0} \in$ Ver. Using (4.18), (4.21), the second statement in (4.13), and the last two statements in Lemma 4.1 as before, we conclude that

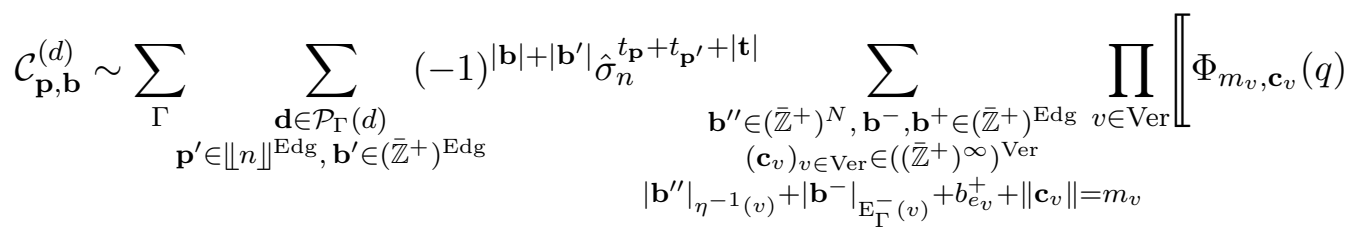

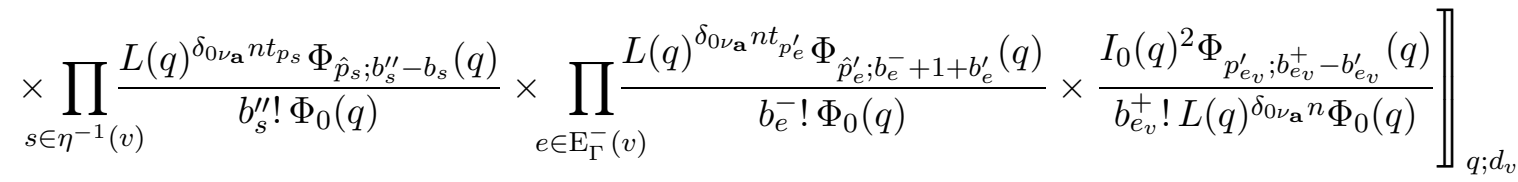

with the last fraction above set to 1 for $v=v_{0}$ and $\mathbf{t} \in\left(\overline{\mathbb{Z}}^{+}\right)^{\text {Ver }}$ defined by (2.46); if an integer $t_{v}$ satisfying (2.46) does not exist for some $v \in$ Ver, the corresponding summand above is defined to be 0 . This confirms (3.6) with $\mathbf{c}_{\mathbf{p}, \mathbf{b}}^{(d, 0)}$ as defined in Section 2.4 (and describes $\mathbf{c}_{\mathbf{p}, \mathbf{b}}^{(d, t)}$ with $t \in \mathbb{Z}^{+}$ as well).

Remark 4.5. The recursion (4.32) and separately the closed formula (4.35) compute the coefficients $\mathcal{C}_{\mathbf{p}, \mathbf{b}}^{(d)}$ in (3.5) and thus provide a straightforward algorithm for computing the equivariant $N$-pointed generating function (3.3). Following the proof of the first statement in Lemma 4.1, the power series $\Psi_{m, \mathbf{c}}(\mathbf{x}, Q)$ and $\Psi_{p ; b}(\mathbf{x}, Q)$ can be computed directly from the power series $\Phi_{b}(\mathbf{x}, q)$ appearing in (4.15). The latter can be computed similarly to the power series $\Phi_{b}(q)$ appearing in Proposition 2.1, see Appendix A. For example, we first find that the power series $\xi$ appearing in (4.15) is described by

$$
\xi \in \mathbf{x} q \cdot \mathbb{Q}\left[\alpha, \mathbf{x}, \sigma_{n-1}(\mathbf{x})^{-1}\right][[q]], \quad \mathbf{x}+\xi^{\prime}(\mathbf{x}, q)=L(\mathbf{x}, q),
$$

where ' denotes $q \frac{\mathrm{d}}{\mathrm{d} q}$ as before $L(\mathbf{x}, q)$ is defined by

$$
L(\mathbf{x}, q) \in \mathbf{x}+\mathbf{x}^{|\mathbf{a}|} q \cdot \mathbb{Q}\left[\alpha, \mathbf{x}, \sigma_{n-1}(\mathbf{x})^{-1}\right]\left[\left[\mathbf{x}^{|\mathbf{a}|-1} q\right]\right], \quad \sigma_{n}(L(\mathbf{x}, q))-q \mathbf{a}^{\mathbf{a}} L(\mathbf{x}, q)^{|\mathbf{a}|}=\sigma_{n}(\mathbf{x}),
$$


with $\sigma_{n}(\cdot)$ defined analogously to (4.8); setting $\alpha=0$ and $\mathbf{x}=1$ above gives (2.2). We then find that

$$
\Phi_{0}(\mathbf{x}, q)=\left(\frac{\mathbf{x} \cdot \sigma_{n-1}(\mathbf{x})}{L(\mathbf{x}, q) \sigma_{n-1}(L(\mathbf{x}, q))-|\mathbf{a}|\left(\sigma_{n}(L(\mathbf{x}, q))-\sigma_{n}(\mathbf{x})\right)}\right)^{1 / 2}\left(\frac{L(\mathbf{x}, q)}{\mathbf{x}}\right)^{(l+1) / 2} ;
$$

setting $\alpha=0$ and $\mathbf{x}=1$ above gives (2.11). This suffices for the $N=3$ case of (3.5).

\section{Proof of Theorem 1}

In this section we prove the bound of Theorem 1 for $d \in \mathbb{Z}^{+}$by considering four separate cases: $|\mathbf{a}|>n$ and $|\mathbf{a}| \leq n$ with $N=1,2,3+$. The first case is fairly straightforward, since there are only finitely many nonzero GW-invariants modulo the string, dilaton, and divisor relations [15, p527]. In the $|\mathbf{a}| \leq n$ cases, we use explicit mirror formulas. For $N=1,2,(2.13)$ and (2.19) reduce Theorem 1 to extracting the coefficients of $w^{b} q^{d}$ from the power series $F(w, q)$ and $F_{p}(w, q)$ defined in (1.4) and (2.18); Corollary 5.3 below presents them in a convenient form. For $N \geq 3$, the coefficients $\mathbf{c}_{\mathbf{p}, \mathbf{b}}^{(d, 0)}$ in Theorem $\mathrm{A}$ must also be suitable bounded. This is done by Proposition 5.4, its proof constitutes most of this section.

We begin by considering the $|\mathbf{a}|>n$ case. Let

$$
d_{\max }=\{d \in \mathbb{Z}:(|\mathbf{a}|-n) d \leq n-4-l\} .
$$

If $d>d_{\max }$, the virtual dimension of $\overline{\mathfrak{M}}_{0,0}\left(X_{\mathbf{a}}, d\right)$ is negative, and so all genus 0 degree $d$ GWinvariants vanish. Thus, we can assume that $d_{\max } \in \mathbb{Z}^{+}$. Let $C \in \mathbb{R}^{+}$be such that

$$
\left|\left\langle b_{1} ! \tau_{b_{1}} H^{c_{1}}, \ldots, b_{N} ! \tau_{b_{N}} H^{c_{N}}\right\rangle_{0, d}^{X_{\mathbf{a}}}\right| \leq C
$$

whenever $b_{s}+c_{s} \geq 2$ for all $s$ or $N \leq d_{\max }$; the number of nonzero invariants of this form is finite. Let $b_{\max }$ be the largest of the sums $b_{1}+\ldots+b_{N}$ for nonzero invariants of this form. It then follows by induction via the dilaton, string, and divisor relations that

$$
\begin{aligned}
\left|\langle b_{1} ! \tau_{b_{1}} H^{c_{1}}, \ldots, b_{N} ! \tau_{b_{N}} H^{c_{N}}, \underbrace{\tau_{0} H^{1}, \ldots, \tau_{0} H^{1}}_{k_{1}}, \underbrace{\tau_{0} H^{0}, \ldots, \tau_{0} H^{0}}_{k_{2}}, \underbrace{\tau_{1} H^{0}, \ldots, \tau_{1} H^{0}}_{k_{3}}\rangle_{0, d}^{X_{\mathbf{a}}}\right| \\
\leq C\left(b_{\max }+d_{\max }\right)^{k_{1}} \cdot \frac{\left(b_{\max }+k_{2}\right) !}{b_{\max } !} \cdot \frac{\left(N+k_{1}+k_{2}+k_{3}\right) !}{\left(N+k_{1}+k_{2}\right) !} \\
\leq C \cdot C^{k_{1}} \cdot 2^{b_{\max }+k_{2}} \cdot\left(N+k_{1}+k_{2}+k_{3}\right) !
\end{aligned}
$$

This implies the bound in Theorem 1 .

In the remainder of this section, we treat the $|\mathbf{a}|<n$ cases.

\subsection{Outline of proof}

By (1.3) and (2.1), the GW-invariant in Theorem 1 is the coefficient of

$$
Q^{d} \mathbf{H}^{\mathbf{p}} \underline{\hbar}^{-\mathbf{b}-\mathbf{1}} \equiv Q^{d} \prod_{s=1}^{s=N} H_{s}^{p_{s}} \hbar_{s}^{-b_{s}-1}, \quad \text { where } \quad p_{s}=n-1-c_{s},
$$


of the right-hand side of the identity in (2.13) if $N=1$, in (2.19) if $N=2$, and in (2.35) if $N \geq 3$. In particular, we need to bound the growth of the coefficients of

$$
e^{-J(q) H / \hbar} H^{p} \frac{F_{p}(H / \hbar, q)}{I_{p-l}(q)} \in \mathbb{Q}[H]\left[\left[\hbar^{-1}, Q\right]\right], \quad \text { where } \quad q e^{\delta_{0 \nu_{\mathbf{a}}} J(q)}=Q / H^{\nu_{\mathbf{a}}} .
$$

By (1.4), (2.14) $-(2.18)$, for every $p \in \mathbb{Z}^{+}$there exists $\hat{F}_{p} \in \mathbb{Q}(w)[[q]]$ such that

$$
e^{-J(q) H / \hbar} H^{p} \frac{F_{p}(H / \hbar, q)}{I_{p-l}(q)}=\hbar^{p} \hat{F}_{p}\left(H / \hbar, Q / \hbar^{\nu \mathbf{a}}\right),
$$

and the coefficient of each power of $q$ is holomorphic at $w=0$.

If $b_{1}+c_{1}=\nu_{\mathbf{a}} d+n-3-l,(1.3),(2.1),(2.13)$, and (5.1) give

$$
\left\langle\tau_{b_{1}} H^{c_{1}}\right\rangle_{0, d}^{X_{\mathbf{a}}}=\llbracket \llbracket \llbracket Z\left(\hbar_{1}, H_{1}, Q\right) \rrbracket_{Q ; d} \rrbracket_{\hbar_{1}^{-1} ; b_{1}+1} \rrbracket_{H_{1} ; p_{1}}=\langle\mathbf{a}\rangle \llbracket \llbracket \hat{F}_{l}(w, q) \rrbracket_{q ; d} \rrbracket_{w ; p_{1}},
$$

where $p_{1}=n-1-c_{1}$ as before. Thus, by Corollary 5.3 below,

$$
\begin{aligned}
\left|\left\langle b_{1} ! \tau_{b_{1}} H^{c_{1}}\right\rangle_{0, d}^{X_{\mathbf{a}}}\right| \leq\langle\mathbf{a}\rangle C_{\mathbf{a}}^{d} \frac{b_{1} !}{\left(\nu_{\mathbf{a}} d\right) !} & \leq\langle\mathbf{a}\rangle C_{\mathbf{a}}^{d}(n-3-l) !\left(\begin{array}{c}
\nu_{\mathbf{a}} d+n-3-l \\
\nu_{\mathbf{a}} d
\end{array}\right) \\
& \leq(n-3-l) !\langle\mathbf{a}\rangle C_{\mathbf{a}}^{d} \cdot 2^{\nu_{\mathbf{a}} d+n-3-l}
\end{aligned}
$$

this confirms the statement of Theorem 1 for $N=1$.

If $b_{1}+c_{1}+b_{2}+c_{2}=\nu_{\mathbf{a}} d+n-3-l,(1.3),(2.1),(2.19)$, and (5.1) give

$$
\begin{gathered}
\sum_{\substack{\delta_{1}+\delta_{2}=1 \\
\delta_{1}, \delta_{2} \geq 0}}\left\langle\tau_{b_{1}+\delta_{1}} H^{c_{1}}, \tau_{b_{2}+\delta_{2}} H^{c_{2}}\right\rangle_{0, d}^{X_{\mathbf{a}}}=\sum_{\substack{\delta_{1}+\delta_{2}=1 \\
\delta_{1}, \delta_{2} \geq 0}} \llbracket \llbracket \llbracket Z(\underline{\hbar}, \mathbf{H}, Q) \rrbracket_{Q ; d}\left\|_{\underline{\hbar}^{-1} ;\left(b_{1}+1+\delta_{1}, b_{2}+1+\delta_{2}\right)}\right\|_{\mathbf{H} ;\left(p_{1}, p_{2}\right)} \\
=\langle\mathbf{a}\rangle \sum_{\substack{d_{1}+d_{2}=d \\
d_{1}, d_{2} \geq 0 \\
\nu_{\mathbf{a}} d_{s} \geq l+1+b_{s}-p_{s}}} \prod_{s=1}^{s=2} \llbracket \llbracket \hat{F}_{\nu_{\mathbf{a}} d_{s}+p_{s}-b_{s}-1}(w, q)\left\|_{q ; d_{s}}\right\|_{w ; p_{s}},
\end{gathered}
$$

with $p_{s}=n-1-c_{s}, \underline{\hbar}=\left(\hbar_{1}, \hbar_{2}\right)$, and $\mathbf{H}=\left(H_{1}, H_{2}\right)$. This gives

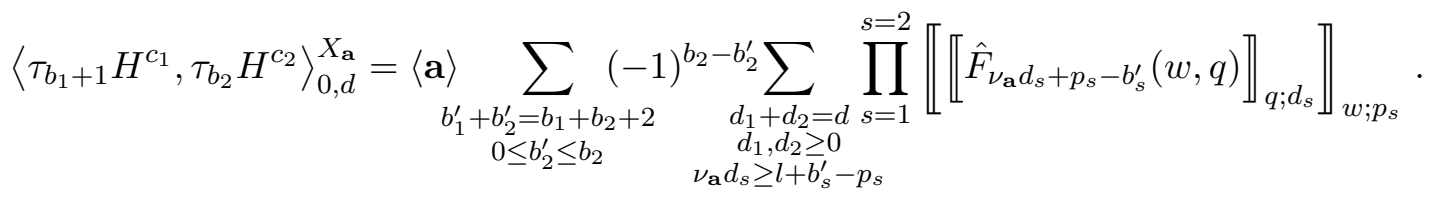

Thus, by Corollary 5.3 below,

$$
\begin{aligned}
\left|\left\langle\left(b_{1}+1\right) ! \tau_{b_{1}+1} H^{c_{1}}, b_{2} ! \tau_{b_{2}} H^{c_{2}}\right\rangle_{0, d}^{X_{\mathbf{a}}}\right| & \leq\langle\mathbf{a}\rangle\left(b_{2}+1\right) C_{\mathbf{a}}^{d} \frac{\left(b_{1}+1\right) ! b_{2} !}{\left(\nu_{\mathbf{a}} d\right) !} \sum_{d_{1}=0}^{d_{1}=d}\left(\begin{array}{c}
\nu_{\mathbf{a}} d \\
\nu_{\mathbf{a}} d_{1}
\end{array}\right) \\
& \leq\langle\mathbf{a}\rangle C_{\mathbf{a}}^{d} \cdot(n-1-l) !\left(\begin{array}{c}
\nu_{\mathbf{a}} d+n-1-l \\
\nu_{\mathbf{a}} d
\end{array}\right) \cdot 2^{\nu_{\mathbf{a}} d} \\
& \leq(n-1-l) !\langle\mathbf{a}\rangle C_{\mathbf{a}}^{d} \cdot 2^{2 \nu_{\mathbf{a}} d+n-1-l} ;
\end{aligned}
$$


this confirms the statement of Theorem 1 for $N=2$.

Finally, we consider the $N \geq 3$ case. For each $p \in \llbracket n \rrbracket_{l}$, let

$$
\hat{F}_{(p)}(w, q)=\frac{\hat{F}_{p}(w, q)}{\prod_{r=p-l+1}^{n-l-1} I_{r}(q)} .
$$

It is sufficient to assume that the tuples $\mathbf{b} \equiv\left(b_{s}\right)_{s \in[N]}$ and $\mathbf{c} \equiv\left(c_{s}\right)_{s \in[N]}$ in the statement of Theorem 1 satisfy

$$
|\mathbf{b}|+|\mathbf{c}|=\nu_{\mathbf{a}} d+n-4-l+N, \quad b_{s}, c_{s} \geq 0, \quad c_{s} \leq n-1-l .
$$

Let $p_{s}=n-1-c_{s}$. If $\mathbf{d}, \mathbf{b}^{\prime} \in\left(\overline{\mathbb{Z}}^{+}\right)^{N}$, define

$$
\mathbf{p}^{\prime}\left(\mathbf{d}, \mathbf{b}^{\prime}\right) \in\left(\overline{\mathbb{Z}}^{+}\right)^{N} \quad \text { by } \quad p_{s}^{\prime}\left(\mathbf{d}, \mathbf{b}^{\prime}\right)=\nu_{\mathbf{a}} d_{s}+p_{s}-b_{s}+b_{s}^{\prime} .
$$

By (1.3), (2.1), (2.35), (2.20), and (5.1),

$$
\left\langle\tau_{b_{1}} H^{c_{1}}, \ldots, \tau_{b_{N}} H^{c_{N}}\right\rangle_{0, d}^{X_{\mathbf{a}}}=\langle\mathbf{a}\rangle \sum_{\substack{0 \leq d^{\prime} \leq d \\ \mathbf{d} \in \mathcal{P}_{N}\left(\bar{d}-d^{\prime}\right) \\ \mathbf{b}^{\prime} \in\left(\overline{\mathbb{Z}}^{+}\right)^{N}}} \mathrm{c}_{\mathbf{p}^{\prime}\left(d^{\prime}, 0\right)}^{\left(\mathbf{d}, \mathbf{b}^{\prime}\right), \mathbf{b}^{\prime}} \prod_{s=1}^{s=N} \llbracket \llbracket \hat{F}_{\left(p_{s}^{\prime}\left(\mathbf{d}, \mathbf{b}^{\prime}\right)\right)}(w, q) \rrbracket_{q ; d_{s}} \rrbracket_{w ; p_{s}} ;
$$

the above summand vanishes unless $l \leq p_{s}^{\prime}\left(\mathbf{d}, \mathbf{b}^{\prime}\right) \leq n-1$ for all $s \in[N]$. Since $\mathrm{c}_{\mathbf{p}^{\prime}, \mathbf{b}^{\prime}}^{\left(d^{\prime}, 0\right)}=0$ unless $\left|\mathbf{b}^{\prime}\right| \leq N-3$, Corollary 5.3 and Proposition 5.4 thus give

$$
\begin{aligned}
& \mid\left\langle b_{1} ! \tau_{b_{1}} H^{c_{1}}, \ldots, b_{N} ! \tau_{b_{N}} H^{c_{N}}\right\rangle_{0, d}^{X_{\mathbf{a}}} \leq\langle\mathbf{a}\rangle N ! C_{\mathbf{a}}^{N+d} \sum_{\substack{0 \leq d^{\prime} \leq d \\
\mathbf{d} \in \mathcal{P}_{N}\left(\bar{d}-d^{\prime}\right)}} \sum_{\substack{\mathbf{b}^{\prime} \in\left(\overline{\mathbb{Z}}^{+}\right)^{N} \\
\left|\mathbf{b}^{\prime}\right| \leq N-3 \\
b_{s}^{\prime} \geq b_{s}-\nu_{\mathbf{a}} d_{s}-p_{s}}} \prod_{s=1}^{s=N}\left(p_{s} ! \frac{b_{s} !}{b_{s}^{\prime} !\left(\nu_{\mathbf{a}} d_{s}\right) ! p_{s} !}\right) \\
& \leq\langle\mathbf{a}\rangle N ! C_{\mathbf{a}}^{N+d} \sum_{\substack{0 \leq d^{\prime} \leq d \\
\mathbf{d} \in \mathcal{P}_{N}\left(d-d^{\prime}\right)}} \sum_{\substack{\mathbf{b}^{\prime} \in\left(\overline{\mathbb{Z}}^{+}\right)^{N} \\
\left|\mathbf{b}^{\prime}\right| \leq N-3 \\
b_{s}^{\prime} \geq b_{s}-\nu_{\mathbf{a}} d_{s}-p_{s}}} \prod_{s=1}^{s=N}\left(n ! 3^{b_{s}}\right) \\
& \leq\langle\mathbf{a}\rangle N ! C_{\mathbf{a}}^{N+d} \cdot(n !)^{N} 3^{\nu_{\mathbf{a}} d+n+N} \cdot\left(\begin{array}{c}
d+N \\
N
\end{array}\right)\left(\begin{array}{c}
N-3+N \\
N
\end{array}\right) \leq N ! C_{\mathbf{a}}^{\prime N+d} \cdot 2^{d+N} \cdot 2^{2 N-3} .
\end{aligned}
$$

This confirms the statement of Theorem 1 for $N \geq 3$.

Remark 5.1. For any non-vanishing summand on the right-hand side of (5.3), $p_{s}^{\prime}\left(\mathbf{d}, \mathbf{b}^{\prime}\right) \leq n-1$ and so $b_{s}+c_{s} \geq \nu_{\mathbf{a}} d_{s}$. Thus, $d_{s}=0$ if $b_{s}+c_{s}<\nu_{\mathbf{a}}$. Since the coefficient of $q^{0}$ in $\hat{F}_{(p)}(w, q)$ is $w^{p}$, it follows that $p_{s}^{\prime}\left(\mathbf{d}, \mathbf{b}^{\prime}\right)=p_{s}$ and $b_{s}^{\prime}=b_{s}$ in such a case. Since $\left|\mathbf{b}^{\prime}\right| \leq N-3$, this implies Theorem 2 .

\subsection{Bounds on the coefficients of generating functions}

In this section, we obtain the bounds on the coefficients of the power series $F_{p}, \hat{F}_{p} \in \mathbb{Q}(w)[[q]]$ defined in (5.1) and (5.2) that are used in the proof of Theorem 1 above. 
Lemma 5.2. There exists $C_{\mathbf{a}} \in \mathbb{R}^{+}$such that

$$
\left|\llbracket \llbracket F_{p^{\prime}}(w, q) \rrbracket_{q ; d} \rrbracket_{w ; \nu_{\mathbf{a}} d-p^{\prime}+p}\right| \leq \frac{C_{\mathbf{a}}^{d}}{\left(\nu_{\mathbf{a}} d\right) !}
$$

for all $p, p^{\prime}=0,1, \ldots, n-1$ and $d \in \overline{\mathbb{Z}}^{+}$.

Proof. By (1.4), (2.14)-(2.16), and (2.18) , it is sufficient to show that there exists $C \in \mathbb{R}^{+}$such that

$$
\left|\llbracket \llbracket F_{0}(w, q) \rrbracket_{q ; d} \rrbracket_{w ; \nu_{\mathbf{a}} d+p}\right|,\left|\llbracket \llbracket F(w, q) \rrbracket_{q ; d} \rrbracket_{w ; \nu_{\mathbf{a}} d-l+p}\right| \leq \frac{C^{d}}{\left(\nu_{\mathbf{a}} d\right) !}
$$

for all $p=0,1, \ldots, n-1$ and $d \in \overline{\mathbb{Z}}^{+}$. Both numbers on the left-hand side vanish for $p<l$ (unless $d, p=0$ in the case of the first number). If $l \leq p<n$,

$$
\begin{aligned}
& \left|\llbracket \llbracket F(w, q) \rrbracket_{q ; d} \rrbracket_{w ; \nu_{\mathbf{a}} d-l+p}\right|=\frac{\prod_{k=1}^{l}\left(a_{k} d\right) !}{(d !)^{n}}||\left|\frac{\prod_{k=1}^{l} \prod_{r=1}^{a_{k} d}\left(1+\left(a_{k} / r\right) w\right)}{\prod_{r=1}^{d}(1+w / r)^{n}} \|_{w ; p-l}\right| \\
& \leq n^{n d} \frac{(|\mathbf{a}| d) !}{(n d) !} \cdot \llbracket \frac{(1+|\mathbf{a}| w)^{(|\mathbf{a}|-l) d}}{(1-w)^{(n-l) d}} \|_{w ; p-l} \\
& \leq \frac{n^{n d}}{\left(\nu_{\mathbf{a}} d\right) !} \sum_{\substack{r+s=p-l \\
r, s \geq 0}}\left(\begin{array}{c}
(n-l) d+r-1 \\
r
\end{array}\right)\left(\begin{array}{c}
(|\mathbf{a}|-l) d \\
s
\end{array}\right)|\mathbf{a}|^{s} \leq \frac{n^{n d}}{\left(\nu_{\mathbf{a}} d\right) !} 2^{(n-l) d+p-l}(|\mathbf{a}|+1)^{(|\mathbf{a}|-l) d} .
\end{aligned}
$$

The first inequality above follows from Stirling's formula [1, Section 15.22],

$$
1<\frac{e^{d}}{\sqrt{2 \pi} d^{d+\frac{1}{2}}} d !<e^{\frac{1}{8 d}} \quad \forall d \in \mathbb{Z}^{+} ;
$$

the following statement uses the Binomial Theorem. The desired bound for $F_{0}(w, q)$ is obtained similarly.

Corollary 5.3. There exists $C_{\mathbf{a}} \in \mathbb{R}^{+}$such that

$$
\left|\llbracket \llbracket \hat{F}_{p^{\prime}}(w, q) \rrbracket_{q ; d} \rrbracket_{w ; p}\right|,\left|\llbracket \llbracket \hat{F}_{\left(p^{\prime}\right)}(w, q) \rrbracket_{q ; d} \rrbracket_{w ; p}\right| \leq \frac{C_{\mathbf{a}}^{d}}{\left(\nu_{\mathbf{a}} d\right) !}
$$

for all $p, p^{\prime}=0,1, \ldots, n-1$ and $d \in \overline{\mathbb{Z}}^{+}$.

Proof. If $\nu_{\mathbf{a}} \geq 2$,

$$
\llbracket \llbracket \hat{F}_{p^{\prime}}(w, q) \rrbracket_{q ; d} \rrbracket_{w ; p}=\llbracket \llbracket F_{p^{\prime}}(w, q) \rrbracket_{q ; d} \rrbracket_{w ; \nu_{\mathbf{a}} d-p^{\prime}+p},
$$

and the claim follows immediately from Lemma 5.2 . If $\nu_{\mathbf{a}}=1$, by (1.5)

$$
\begin{gathered}
\llbracket \llbracket \hat{F}_{p^{\prime}}(w, q) \rrbracket_{q ; d} \rrbracket_{w ; p}=\sum_{\substack{d_{1}+d_{2}=d \\
d_{1}, d_{2} \geq 0}} \frac{(-\mathbf{a} !)^{d_{1}}}{d_{1} !} \llbracket \llbracket F_{p^{\prime}}(w, q) \rrbracket_{q ; d_{2}} \rrbracket_{w ; d_{2}-p^{\prime}+p} \\
\Longrightarrow \quad\left|\llbracket \llbracket \hat{F}_{p^{\prime}}(w, q) \rrbracket_{q ; d} \rrbracket_{w ; p}\right| \leq \frac{\left(\mathbf{a} !+C_{\mathbf{a}}\right)^{d}}{d !},
\end{gathered}
$$


where $C_{\mathbf{a}}$ is as in Lemma 5.2. Finally, suppose $\nu_{\mathbf{a}}=0$. Define

$$
\tilde{J} \in Q \cdot \mathbb{Q}[[Q]] \quad \text { by } \quad q=Q e^{\tilde{J}(Q)} .
$$

By Lemma 5.2 ,

$$
\left|\llbracket I_{0}(q) \rrbracket_{q ; d}\right|,\left|\llbracket I_{1}(q) \rrbracket_{q ; d}\right|, \ldots,\left|\llbracket I_{n-1}(q) \rrbracket_{q ; d}\right|,\left|\llbracket J(q) \rrbracket_{q ; d}\right| \leq C^{d} \Longrightarrow\left|\llbracket \tilde{J}(q) \rrbracket_{q ; d}\right| \leq C^{\prime d} ;
$$

the last implication follows from the Inverse Function Theorem. Since

$$
\llbracket \hat{F}_{p^{\prime}}(w, Q) \rrbracket_{w ; p}=\sum_{\substack{p_{1}+p_{2}=p-p^{\prime} \\ p_{1}, p_{2} \geq 0}} \frac{\tilde{J}(Q)^{p_{1}}}{p_{1} !} \frac{\llbracket F_{p^{\prime}}(w, q) \rrbracket_{w ; p_{2}}}{\prod_{r=p-l}^{n-l-1} I_{r}(q)}
$$

the claim again follows from Lemma 5.2 .

\subsection{Bounds on the structure constants in Theorem $\mathrm{A}$}

In this section, we obtain an upper bound for the coefficients $\mathrm{c}_{(\mathbf{p}, \mathbf{b})}^{(d, 0)}$ in Theorem $\mathrm{A}$. This is one the two key ingredients in the proof of Theorem 1

Proposition 5.4. If $n, N \in \mathbb{Z}^{+}$with $N \geq 3$ and $\mathbf{a} \in\left(\mathbb{Z}^{+}\right)^{l}$ with $|\mathbf{a}| \leq n$, there exists $C_{\mathbf{a}} \in \mathbb{R}^{+}$such that

$$
\left|\mathbf{c}_{\mathbf{p}, \mathbf{b}}^{(d, 0)}\right| \leq \frac{N !}{\mathbf{b} !} C_{\mathbf{a}}^{N+d} \quad \forall d \in \overline{\mathbb{Z}}^{+}, \mathbf{p} \in \llbracket n \rrbracket^{N}, \mathbf{b} \in\left(\overline{\mathbb{Z}}^{+}\right)^{N} .
$$

Lemma 5.5. If $n \in \mathbb{Z}^{+}, a \in(0, n)$, and $L \in 1+q \mathbb{Q}[[q]]$ is defined by

$$
L(q)^{n}-q L(q)^{a}=1
$$

then there exists $C_{a} \in \mathbb{R}^{+}$such that

$$
\left|\llbracket \frac{L(q)^{1-n+k}}{(1-q)^{\delta}\left(a+(n-a) L(q)^{n}\right)^{k^{\prime}}} \rrbracket_{q ; d}\right| \leq C_{a} \quad \forall k, k^{\prime} \in \overline{\mathbb{Z}}^{+}, k \leq 2 n^{2}, k^{\prime} \leq 2 n+1, \delta=0,1 .
$$

Proof. Let $\nu=n-a$. We show that (5.5) defines a holomorphic map $q \longrightarrow L(q)$ on a neighborhood of the closed unit disk $\bar{D} \subset \mathbb{C}$ such that

$$
L(q), a+\nu L(q) \neq 0 \quad \forall q \in \bar{D} .
$$

Thus, the radius of convergence of the Cauchy series around $q=0$ for the holomorphic function

$$
q \longrightarrow \frac{L(q)^{k}}{\left(a+\nu L(q)^{n}\right)^{k^{\prime}}}
$$

is greater than 1 . Let

$$
S=\left\{(q, z) \in \mathbb{C}^{2}: z^{n}-q z^{a}=1\right\}
$$


Since the differential of the defining equation is surjective for $z \neq 0, S$ is a smooth curve in $\mathbb{C}^{2}$. The projection map $\pi_{1}: S \longrightarrow \mathbb{C}$ to the first coordinate is an $n$-fold cover branched at the points $(q, z) \in S$ such that

$$
\begin{aligned}
n z^{n-1}-q a z^{a-1}=0 \quad \Longrightarrow \quad q=\frac{n}{a} z^{\nu} & \Longrightarrow z^{n}=-\frac{a}{\nu} \\
& \Longrightarrow|q|=\frac{n}{a} \cdot\left(\frac{a}{\nu}\right)^{\nu / n}>\left(\frac{n}{a}\right)^{a / n}>1 .
\end{aligned}
$$

Thus, $\pi_{1}$ is an unramified cover of an open neighborhood $U$ of $\bar{D}$, and its restriction to the component of $\pi^{-1}(0)$ containing $(0,1)$ induces a holomorphic map

$$
U \longrightarrow \mathbb{C}, \quad q \longrightarrow L(q)
$$

solving (5.5). It is immediate from (5.5) that $L(q) \neq 0$ for all $q$, if $a>0$. On the other hand,

$$
\begin{aligned}
1+\frac{\nu}{n} q L(q)^{a}=0 \quad \Longrightarrow \quad q=-\frac{n}{\nu} L(q)^{-a} & \Longrightarrow L(q)^{n}=-\frac{a}{\nu} \\
& \Longrightarrow|q|=\frac{n}{\nu} \cdot\left(\frac{\nu}{a}\right)^{a / n}>\left(\frac{n}{\nu}\right)^{\nu / n}>1,
\end{aligned}
$$

as claimed.

Lemma 5.6. Let $\Phi_{0}, \Phi_{1}, \ldots \in \mathbb{Q}[[q]]$ be as in Proposition [2.1. There exists $C_{\mathbf{a}} \in \mathbb{R}^{+}$such that

$$
\left|\llbracket \frac{\Phi_{b}(q)}{\Phi_{0}(q)} \rrbracket_{q ; d}\right| \leq b ! C_{\mathbf{a}}^{b} \llbracket\left(1-C_{\mathbf{a}} q\right)^{-b} \rrbracket_{q ; d} \quad \forall b, d \in \overline{\mathbb{Z}}^{+} .
$$

Proof. For $k=1,2, \ldots, n$, define

$$
\widetilde{\mathfrak{L}}_{k}: \mathbb{Q}[[q]] \longrightarrow \mathbb{Q}[[q]] \quad \text { by } \quad \widetilde{\mathfrak{L}}_{k}(\Phi)=\frac{1}{L(q)^{k-1} \Phi_{0}(q)\left(|\mathbf{a}|+\nu_{\mathbf{a}} L(q)^{n}\right)} \mathfrak{L}_{k}\left(\Phi_{0} \Phi\right),
$$

with $\mathfrak{L}_{k}$ and $\Phi_{0}$ given by (2.7) and (2.11), respectively. These differential operators are of the form

$$
\widetilde{\mathfrak{L}}_{k}=\sum_{i=0}^{i=k} \tilde{h}_{k, k-i}(q) D^{i} \quad \text { with } \quad \tilde{h}_{k, i} \in \mathbb{Q}[[q]] .
$$

Note that by (2.2) and

$$
\frac{L^{\prime}}{L}=\frac{L^{n}-1}{|\mathbf{a}|+\nu_{\mathbf{a}} L^{n}}=\frac{\mathbf{a}^{\mathbf{a}} q L(q)^{|\mathbf{a}|}}{|\mathbf{a}|+\nu_{\mathbf{a}} L^{n}} .
$$

We now consider three separate cases.

(1) Suppose $0<|\mathbf{a}|<n$. We show that there exists $C_{\mathbf{a}} \in \mathbb{R}^{+}$such that

$$
\left|\llbracket \frac{\Phi_{b}(q)}{\Phi_{0}(q)} \rrbracket_{q ; d}\right| \leq b ! C_{\mathbf{a}}^{b} \llbracket\left(1-\mathbf{a}^{\mathbf{a}} q\right)^{-b} \rrbracket_{q ; d} \quad \forall b, d \in \overline{\mathbb{Z}}^{+} .
$$

By (2.11) and (5.7), for each $j \in \mathbb{Z}^{+}$there exists $p_{j} \in \mathbb{Q}[u]$ such that

$$
\frac{D^{j} \Phi_{0}}{\Phi_{0}}=\frac{\left(L^{n}-1\right) p_{j}\left(L^{n}\right)}{\left(|\mathbf{a}|+\nu_{\mathbf{a}} L^{n}\right)^{2 j}}=\frac{\mathbf{a}^{\mathbf{a}} q L(q)^{|\mathbf{a}|} p_{j}\left(L^{n}\right)}{\left(|\mathbf{a}|+\nu_{\mathbf{a}} L^{n}\right)^{2 j}}, \quad \operatorname{deg} p_{j} \leq 2 j-1 .
$$


By (2.5), for each $j \in \mathbb{Z}^{+}$there exist $p_{m, j} \in \mathbb{Q}[u]$ such that

$$
\mathcal{H}_{m, j}(u)=\frac{(u-1) p_{m, j}(u)}{\left(|\mathbf{a}|+\nu_{\mathbf{a}} u\right)^{2 j-1}}, \quad \operatorname{deg} p_{m, j} \leq 2 j-2,
$$

where $\mathcal{H}_{m, j} \in Q(u)$ is the function defined in Section 2.1. Thus, by (2.7) and (5.9), there exist $\tilde{p}_{k, i} \in \mathbb{Q}[u]$ such that

$$
\tilde{h}_{k, i}=\frac{1}{L^{k-1}} \cdot \frac{\left(q L^{|\mathbf{a}|}\right)^{\delta_{i, k}} \tilde{p}_{k, i}\left(L^{n}\right)}{\left(|\mathbf{a}|+\nu_{\mathbf{a}} L^{n}\right)^{2 i+1}}, \quad \operatorname{deg} \tilde{p}_{k, i} \leq 2 i+1-\delta_{i, k}
$$

Let $C \geq 1$ be the maximum of the absolute values of the coefficients of the polynomials $(2 i+1) \tilde{p}_{k, i}$, with $i=0,1, \ldots, k$ and $k=2,3, \ldots, n$. Thus,

$$
\left|\llbracket\left(1-\mathbf{a}^{\mathbf{a}} q\right)^{-b} \tilde{h}_{k, i}(q) \rrbracket_{q ; d}\right| \leq C C_{|\mathbf{a}|} \llbracket q^{\delta_{i, k}}\left(1-\mathbf{a}^{\mathbf{a}} q\right)^{-b} \rrbracket_{q ; d} \quad \forall k=2,3, \ldots, n, b \in \mathbb{Z}^{+},
$$

where $C_{|\mathbf{a}|}$ is as in Lemma 5.5. We show that (5.8) holds with

$$
C_{\mathbf{a}}=n^{2} C C_{|\mathbf{a}|} \mathbf{a}^{\mathbf{a}}
$$

This is indeed the case for $b=0$. Suppose $b^{*} \geq 1$ and the bound holds for all $b<b^{*}$. By (2.10), (5.6), (5.10), and the inductive assumption,

$$
\begin{aligned}
\left|\llbracket D\left(\frac{\Phi_{b^{*}}(q)}{\Phi_{0}(q)}\right) \rrbracket_{q ; d}\right| & \leq \sum_{k=2}^{k=n}\left|\llbracket \widetilde{\mathfrak{L}}_{k}\left(\frac{\Phi_{b^{*}-k+1}(q)}{\Phi_{0}(q)}\right) \rrbracket_{q ; d}\right| \\
& \leq n^{2} C C_{|\mathbf{a}|} \cdot C_{\mathbf{a}}^{b^{*}-1} b^{*} ! b^{*}\left(\mathbf{a}^{\mathbf{a}}\right)^{2} \llbracket q\left(1-\mathbf{a}^{\mathbf{a}} q\right)^{-b^{*}-1} \rrbracket_{q ; d} .
\end{aligned}
$$

Integrating this inequality, we find that (5.8) holds for $b=b^{*}$ as well.

(2) Suppose next that $|\mathbf{a}|=n$. We show that (5.8) still holds. Since $n D L / L=\left(L^{n}-1\right)$ in this case, for each $j \in \mathbb{Z}^{+}$there exists $p_{j} \in \mathbb{Q}[u]$ such that

$$
\frac{D^{j} \Phi_{0}}{\Phi_{0}}=\left(L^{n}-1\right) p_{j}\left(L^{n}\right)=\mathbf{a}^{\mathbf{a}} q L(q)^{n} p_{j}\left(L^{n}\right), \quad \operatorname{deg} p_{j} \leq j-1
$$

On the other hand, by (2.5) for each $j \in \mathbb{Z}^{+}$there exist $p_{m, j} \in \mathbb{Q}[u]$ such that

$$
\mathcal{H}_{m, j}(u)=(u-1) p_{m, j}(u), \quad \operatorname{deg} p_{m, j} \leq j-1 .
$$

It follows that there exists $\tilde{p}_{k, i} \in \mathbb{Q}[u]$ such that

$$
\tilde{h}_{k, i}=\frac{1}{L^{k-1}}\left(q L(q)^{n}\right)^{\delta_{i, k}} \tilde{p}_{k, i}\left(L^{n}\right), \quad \operatorname{deg} \tilde{p}_{k, i} \leq i-\delta_{i, k} .
$$

Let $C \geq 1$ be the maximum of the absolute values of the coefficients of the polynomials $(i+1) \tilde{p}_{k, i}$, with $i=0,1, \ldots, k$ and $k=2,3, \ldots, n$. Thus,

$$
\left|\llbracket\left(1-\mathbf{a}^{\mathbf{a}} q\right)^{-b} \tilde{h}_{k, i}(q) \rrbracket_{q ; d}\right| \leq C \llbracket q^{\delta_{i, k}}\left(1-\mathbf{a}^{\mathbf{a}} q\right)^{-b-k} \rrbracket_{q ; d} \quad \forall k=2,3, \ldots, n, b \in \mathbb{Z}^{+} ;
$$


see (2.3). The same inductive argument as at the end of (1) now shows that (5.8) holds with $C_{\mathbf{a}}=n^{2} C \mathbf{a}^{\mathbf{a}}$.

(3) Finally, suppose $|\mathbf{a}|=0$, i.e. $\mathbf{a}=()$. We show that there exist $C_{\emptyset}, C_{b, r} \in \mathbb{Q}$ for $b, r \in \overline{\mathbb{Z}}^{+}$such that

$$
\frac{\Phi_{b}}{\Phi_{0}}=\sum_{r=0}^{(n+1) b} C_{b, r} L^{-r}, \quad \sum_{r=0}^{(n+1) b}\left|C_{b, r}\right| \leq b ! C_{\emptyset}^{b} \quad \forall b \in \mathbb{Z}^{+} .
$$

This implies the claim, since

$$
\left|\llbracket L(q)^{-r} \rrbracket_{q ; d}\right| \leq\left|\llbracket L(q)^{-2 n b} \rrbracket_{q ; d}\right|=\left|\left(\begin{array}{c}
-2 b \\
d
\end{array}\right)\right|=\left(\begin{array}{c}
2 b+d-1 \\
d
\end{array}\right) \leq 2^{2 b+d} \leq 2^{2 b} \llbracket(1-2 q)^{b} \rrbracket_{q ; d}
$$

for all $r \leq 2 n b$ and $b \in \mathbb{Z}^{+}$.

Since $n D L / L=\left(1-L^{-n}\right)$ in this case, there exist $C_{r ; i}^{(j)} \in \mathbb{Q}$ such that

$$
D^{i} L^{-r}=L^{-r} \frac{D L}{L} \sum_{j=0}^{i-1}(r+n j) C_{r ; i}^{(j)} L^{-n j}, \quad \sum_{j=0}^{i-1}\left|C_{r ; i}^{(j)}\right| \leq 2^{i-1} \prod_{j=0}^{i-2} \frac{r+n j}{n} \quad \forall r \in \overline{\mathbb{R}}^{+}, i \in \mathbb{Z}^{+}
$$

On the other hand, by (2.5) for each $j \in \mathbb{Z}^{+}$there exist $p_{m, j} \in u \cdot \mathbb{Q}[u]$

$$
\mathcal{H}_{m, j}(u)=(u-1) p_{m, j}(1 / u), \quad \operatorname{deg} p_{m, j} \leq j .
$$

It follows that there exist $\tilde{p}_{k, i} \in \mathbb{Q}[u]$ such that

$$
\tilde{h}_{k, i}=\frac{1}{L^{k-1}} \tilde{p}_{k, i}\left(L^{-n}\right)\left(\frac{D L}{L}\right)^{\delta_{i, k}}, \quad \operatorname{deg} \tilde{p}_{k, i} \leq i-\delta_{i, k} \quad \forall i \in \overline{\mathbb{Z}}^{+} .
$$

Thus, there exist $\tilde{C}_{r ; k}^{(j)} \in \mathbb{Q}$ such that

$$
\tilde{\mathfrak{L}}_{k} L^{-r}=L^{-r-k} D L \sum_{j=0}^{k-1}(r+n j+1) \tilde{C}_{r ; k}^{(j)} L^{-n j}, \quad \sum_{j=0}^{k-1}\left|\tilde{C}_{r ; k}^{(j)}\right| \leq 2^{k} C \prod_{j=1}^{k-1} \frac{r+n j}{n}
$$

for all $r \in \overline{\mathbb{R}}^{+}$and $k \in \mathbb{Z}^{+}$, where $C \geq 1$ is the maximum of the absolute values of the coefficients of the polynomials $(k+1) \tilde{p}_{k, i}$ with $i=0,1, \ldots, k$ and $k=1,2, \ldots, n$. We show that (5.14) holds with

$$
C_{\emptyset}=4\left(\frac{2 n+2}{n}\right)^{n} C
$$

This is indeed the case for $b=0$. Suppose $b^{*} \geq 1$ and the claim holds for all $b<b^{*}$. By (2.10), the 
inductive assumption, and (5.16), there exist $C_{b^{*}, r}^{\prime} \in \mathbb{Q}$ such that

$$
\begin{aligned}
D\left(\frac{\Phi_{b^{*}}}{\Phi_{0}}\right) & =-\sum_{k=2}^{k=n} \widetilde{\mathfrak{L}}_{k}\left(\frac{\Phi_{b^{*}-k+1}}{\Phi_{0}}\right)=-\frac{D L}{L} \sum_{r=1}^{(n+1) b^{*}} r C_{b^{*}, r} L^{-r} \\
\sum_{r=1}^{(n+1) b^{*}}\left|C_{b^{*}, r}\right| & \leq C \sum_{k=2}^{n} \sum_{r=0}^{(n+1)} \sum_{j=0}^{\left(b^{*}-1+k\right)}\left|\tilde{C}_{r ; k}^{(j)}\right|\left|C_{b^{*}-k+1, r}\right| \\
& \leq C \sum_{k=2}^{n}\left(2^{k} \prod_{j=1}^{k-1}\left(\frac{(n+1)\left(b^{*}-k+1\right)+n j}{n}\right) \cdot\left(b^{*}-k+1\right) ! C_{\emptyset}^{b^{*}-k+1}\right) \\
& \leq 2 C C_{\emptyset}^{b^{*}-1} \sum_{k=2}^{n}\left(\left(2 \frac{(n+1)}{n}\right)^{k-1} \prod_{j=1}^{k-1}\left(b^{*}-k+1+j\right) \cdot\left(b^{*}-k+1\right) !\right) \leq \frac{C_{\emptyset}^{b^{*}}}{2} b^{*} !
\end{aligned}
$$

Thus, integrating (5.17) and using $\Phi_{b^{*}} \in q \cdot \mathbb{Q}[[q]]$, we find that (5.14) holds for $b=b^{*}$ as well.

Remark 5.7. The above arguments are based on the fact that all coefficients of $(1-q)^{-\alpha}$ are nonnegative (actually positive) if $\alpha>0$, non-decreasing with $\alpha$, are at least as large in the absolute values as the coefficients of $(1 \pm q)^{\alpha}$, and non-decreasing with $d$ if $\alpha \geq 1$.

Corollary 5.8. Let $\Phi_{p ; b}, \Phi_{m, \mathbf{c}}(q) \in \mathbb{Q}[[q]]$ be as in (2.21) and (2.30). There exists $C_{\mathbf{a}} \in \mathbb{R}^{+}$such that

$$
\begin{aligned}
& \left|\llbracket \frac{\Phi_{p ; b}(q)}{\Phi_{0}(q)} \rrbracket_{q ; d}\right| \leq b ! C_{\mathbf{a}}^{b} \llbracket\left(1-C_{\mathbf{a}} q\right)^{-b-1} \rrbracket_{q ; d} \quad \forall b, d \in \overline{\mathbb{Z}}^{+}, p \in \llbracket n \Downarrow ; \\
& \left|\llbracket \Phi_{m, \mathbf{c}}(q) \rrbracket_{q ; d}\right| \leq \frac{(m+|\mathbf{c}|) !}{|\mathbf{c}| !}\left(\begin{array}{c}
|\mathbf{c}| \\
\mathbf{c}
\end{array}\right) \prod_{r=1}^{\infty}\left(\frac{1}{r+1}\right)^{c_{r}} C_{\mathbf{a}}^{\|\mathbf{c}\|} \llbracket\left(1-C_{\mathbf{a}} q\right)^{-\|\mathbf{c}\|-1} \rrbracket_{q ; d} \quad \forall m, d \in \overline{\mathbb{Z}}^{+}, \mathbf{c} \in\left(\overline{\mathbb{Z}}^{+}\right)^{\infty} .
\end{aligned}
$$

Proof. It is sufficient to obtain the first bound for the power series $\hat{\Phi}_{p ; b} \in \mathbb{Q}[[q]],-l \leq p \leq n-1-l$, defined in (2.22). If $0<|\mathbf{a}|<n$, it follows by induction on $b \in \overline{\mathbb{Z}}^{+}$and $p$ (from 0 up to $n-1-l$ and down to $-l$ ) from Lemma 5.6, the $j=1$ case of (5.9), and Lemma 5.5. For $|\mathbf{a}|=n$, Lemma 5.2 implies that there exists $C \in \mathbb{R}^{+}$such that

$$
\left|\llbracket I_{0}(q)^{k_{0}} I_{1}(q)^{k_{1}} \ldots I_{n-l}(q)^{k_{n-l}} \rrbracket_{q ; d}\right| \leq C^{d} \quad \forall d \in \overline{\mathbb{Z}}^{+}, k_{0}, k_{1}, \ldots, k_{n-l} \in\{0, \pm 1\} .
$$

By induction on $b$ and $|p|$ (with the base case being Lemma 5.6) along with (2.3) and the $j=1$ case of (5.11), this implies that

$$
\left|\llbracket \frac{\hat{\Phi}_{l+p ; b}(q)}{\Phi_{0}(q)} \rrbracket_{q ; d}\right| \leq C_{\mathbf{a} ; p}^{b} b ! \llbracket\left(1-C_{\mathbf{a} ; p} q\right)^{-b-|p| / n} \rrbracket_{q ; d} \quad \forall b, d \in \overline{\mathbb{Z}}^{+},
$$

for some $C_{\mathbf{a} ; p} \in \mathbb{R}^{+}$. The same estimate holds if $|\mathbf{a}|=0$, by Lemma 5.6 and (2.3). The second bound follows directly from Lemma 5.6 and (2.11), along with Lemma 5.5 if $0<|\mathbf{a}|<n$ and (5.18) if $|\mathbf{a}|=n$.

Proof of Proposition 5.4. By Corollary [5.8, the absolute value of each nonzero factor $\llbracket \cdot \rrbracket$ in (2.47) is bounded above by

$$
\begin{gathered}
\frac{\left(m_{v}+\left|\mathbf{c}_{v}\right|\right) !}{\left|\mathbf{c}_{v}\right| !}\left(\begin{array}{c}
\left|\mathbf{c}_{v}\right| \\
\mathbf{c}_{v}
\end{array}\right) \prod_{r=1}^{\infty}\left(\frac{1}{r+1}\right)^{c_{v ; r}} \prod_{s \in \eta^{-1}(v)} \frac{1}{b_{s} !} \cdot \prod_{e \in \mathrm{E}_{\Gamma}^{-}(v)} \frac{\left(b_{e}^{-}+1+b_{e}^{\prime}\right) !}{b_{e}^{-} !} \cdot \frac{\left(b_{e_{v}}^{+}-b_{e_{v}}^{\prime}\right) !}{b_{e_{v}}^{+} !} C_{\mathbf{a}}^{\Delta_{v}\left(\mathbf{b}^{\prime}\right)} \llbracket\left(1-C_{\mathbf{a}} q\right)^{-\Delta_{v}\left(\mathbf{b}^{\prime}\right)} \rrbracket_{q ; d_{v}} \\
\text { where } \quad \Delta_{v}\left(\mathbf{b}^{\prime}\right)=4 m_{v}+8-|\mathbf{b}|_{\eta^{-1}(v)}+\left|\mathbf{b}^{\prime}\right|_{\mathrm{E}_{\Gamma}^{-}(v)}-b_{e_{v}}^{\prime}
\end{gathered}
$$


Thus, by (2.45), the absolute value of each nonzero summand (product of factors over $v \in$ Ver) in (2.47) is bounded above by

$$
\frac{C_{\mathbf{a}}^{8 N} \llbracket\left(1-C_{\mathbf{a}} q\right)^{-8 N} \rrbracket_{q ; d}}{\mathbf{b} !} \prod_{v \in \operatorname{Ver}}\left(\frac{\left(m_{v}+\left|\mathbf{c}_{v}\right|\right) !}{\left|\mathbf{c}_{v}\right| !}\left(\begin{array}{c}
\left|\mathbf{c}_{v}\right| \\
\mathbf{c}_{v}
\end{array}\right) \prod_{r=1}^{\infty}\left(\frac{1}{r+1}\right)^{c_{v ; r}}\right) \cdot \prod_{e \in \operatorname{Edg}} \frac{\left(b_{e}^{-}+1+b_{e}^{\prime}\right) !\left(b_{e}^{+}-b_{e}^{\prime}\right) !}{b_{e}^{-} ! b_{e}^{+} !} .
$$

Note that

$$
\begin{aligned}
\sum_{b_{e}^{-}+b_{e}^{+}=b_{e}^{ \pm}} \frac{\left(b_{e}^{-}+1+b_{e}^{\prime}\right) !\left(b_{e}^{+}-b_{e}^{\prime}\right) !}{b_{e}^{-} ! b_{e}^{+} !} \leq \sum_{b_{e}^{-}+b_{e}^{+}=b_{e}^{ \pm}} \frac{\left(b_{e}^{-}+1+b_{e}^{+}\right) !}{b_{e}^{-} ! b_{e}^{+} !} & =\left(b_{e}^{ \pm}+1\right) \sum_{b_{e}^{-}+b_{e}^{+}=b_{e}^{ \pm}}\left(\begin{array}{c}
b_{e}^{-}+b_{e}^{+} \\
b_{e}^{-}
\end{array}\right) \\
& =\left(b_{e}^{ \pm}+1\right) 2^{b_{e}^{ \pm}} \leq 4^{b_{e}^{ \pm}} .
\end{aligned}
$$

Since each tuple $\mathbf{b}^{\prime \prime}$ is a partition of $N-3-|\operatorname{Edg}|-\left|\mathbf{b}^{-}\right|-\left|\mathbf{b}^{+}\right|-\|\mathbf{c}\|$ into $N$ ordered parts, where

$$
\|\mathbf{c}\|=\sum_{v \in \operatorname{Ver}}\left\|\mathbf{c}_{v}\right\|
$$

the number of such tuples with $\left|\mathbf{b}^{-}\right|+\left|\mathbf{b}^{+}\right|$and $\|\mathbf{c}\|$ fixed is at most

$$
\left(\begin{array}{c}
N-3-|\operatorname{Edg}|-\left|\mathbf{b}^{-}\right|-\left|\mathbf{b}^{+}\right|-\|\mathbf{c}\|+N-1 \\
N-1
\end{array}\right) \leq 2^{2(N-2)-\left|\mathbf{b}^{-}\right|-\left|\mathbf{b}^{+}\right|-\|\mathbf{c}\|} .
$$

Thus, the absolute value of the sum in (2.47) with $\Gamma,\left(\mathbf{p}^{\prime}, \mathbf{b}^{\prime}, \mathbf{t}\right)$, and $\mathbf{c}$ fixed is bounded above by

$$
\frac{C_{\mathbf{a}}^{\prime 8 N} \llbracket\left(1-C_{\mathbf{a}} q\right)^{-8 N} \rrbracket_{q ; d}}{\mathbf{b} !} 2^{-\|\mathbf{c}\|} \prod_{v \in \mathrm{Ver}}\left(\frac{\left(m_{v}+\left|\mathbf{c}_{v}\right|\right) !}{\left|\mathbf{c}_{v}\right| !}\left(\begin{array}{c}
\left|\mathbf{c}_{v}\right| \\
\mathbf{c}_{v}
\end{array}\right) \prod_{r=1}^{\infty}\left(\frac{1}{r+1}\right)^{c_{v ; r}}\right) .
$$

Since $|1-2 \ln 2|<1$, by the Binomial Theorem

$$
\begin{aligned}
& \sum_{(\mathbf{c})_{v \in \mathrm{Ver}} \in\left((\overline{\mathbb{Z}}+)^{\infty}\right) \mathrm{Ver}} 2^{-\|\mathbf{c}\|} \prod_{v \in \mathrm{Ver}}\left(\frac{\left(m_{v}+\left|\mathbf{c}_{v}\right|\right) !}{m_{v} !}\left(\begin{array}{c}
\left|\mathbf{c}_{v}\right| \\
\mathbf{c}_{v}
\end{array}\right) \prod_{r=1}^{\infty}\left(\frac{1}{r+1}\right)^{c_{v} ; r}\right) \\
& =\left.\prod_{v \in \mathrm{Ver}}\left(1-\sum_{r=1}^{\infty} \frac{w^{r}}{r+1}\right)^{-m_{v}-1}\right|_{w=1 / 2}=\left.\left(2+\frac{\ln (1-w)}{w}\right)^{-(N-2)}\right|_{w=1 / 2}=(2(1-\ln 2))^{N-2}
\end{aligned}
$$

Since $b_{e}^{\prime} \leq b_{e}^{+}$for $e \in$ Edg and nonzero summands in (2.47), $\left|\mathbf{b}^{\prime}\right| \leq N-3-|\operatorname{Edg}|$. The number of such tuples is

$$
\left(\begin{array}{c}
N-3-|\operatorname{Edg}|+|\operatorname{Edg}| \\
|\mathrm{Edg}|
\end{array}\right) \leq 2^{N-3}
$$

Thus, the absolute value of the contribution of each trivalent $N$-marked tree $\Gamma$ to $\mathrm{c}_{\mathbf{p}, \mathbf{b}}^{(d, 0)}$ is bounded above by

$$
\frac{\tilde{C}_{\mathbf{a}}^{N}}{\mathbf{b} !}\left(\begin{array}{c}
-8 N \\
d
\end{array}\right) C_{\mathbf{a}}^{d} \cdot \prod_{v \in \mathrm{Ver}} m_{v} !=\frac{\tilde{C}_{\mathbf{a}}^{N}}{\mathbf{b} !}\left(\begin{array}{c}
8 N+d-1 \\
d
\end{array}\right) C_{\mathbf{a}}^{d} \cdot \prod_{v \in \mathrm{Ver}} m_{v} ! \leq \frac{\tilde{C}_{\mathbf{a}}^{N}}{\mathbf{b} !} 2^{8 N+d} C_{\mathbf{a}}^{d} \cdot \prod_{v \in \mathrm{Ver}} m_{v} ! .
$$

Combining this with Lemma 5.10 below, we obtain the claimed bound for $\mathbf{c}_{\mathbf{p}, \mathbf{b}}^{(d, 0)}$. 
Remark 5.9. In the $|\mathbf{a}|=0$ case (projective space), a bound of the form $(N ! / \mathbf{b} !) C^{N-3-|\mathbf{b}|}$ can be obtained using the last description of $c_{\mathbf{p}, \mathbf{b}}^{(d, 0)}$ in Section 2.4 and (5.14).

Lemma 5.10. There exist $C \in \mathbb{R}^{+}$such that

$$
a_{N-1} \equiv \sum_{\Gamma} \prod_{v \in \text { Ver }} m_{v} ! \leq C^{N} N ! \quad \forall N \geq 3
$$

where the sum is taken over all trivalent $N$-marked trees.

Proof. Let $a_{1}=1$ and

$$
f(q)=\sum_{N=1}^{\infty} \frac{a_{N}}{N !} q^{N} \in \mathbb{Q}[[q]] .
$$

Considering the vertex of an $(N+1)$-marked tree $\Gamma$ to which the last marked point is attached, we observe that

$$
\begin{aligned}
a_{N} & =\sum_{k=2}^{k=N} \frac{1}{k !} \sum_{\left(N_{1}, \ldots, N_{k}\right) \in \mathcal{P}_{k}(N)}\left(\begin{array}{c}
N \\
N_{1}, \ldots, N_{k}
\end{array}\right)(k-2) ! a_{N_{1}} \ldots a_{N_{k}} \\
& =N ! \sum_{k=2}^{k=N}\left(\frac{1}{k-1}-\frac{1}{k}\right) \sum_{\left(N_{1}, \ldots, N_{k}\right) \in \mathcal{P}_{k}(N)} \frac{a_{N_{1}}}{N_{1} !} \ldots \frac{a_{N_{k}}}{N_{k} !} .
\end{aligned}
$$

This recursion is equivalent to the condition that

$$
f(q)=q+f(q)+(f(q)-1) \sum_{k=1}^{\infty} \frac{f(q)^{k}}{k} \Longleftrightarrow \quad(1-f(q)) \ln (1-f(q))=-q .
$$

By the Inverse Function Theorem, $f(q)$ is an analytic function on a neighborhood of $q=0$ and so $a_{N} / N ! \leq C^{N}$ for some $C \in \mathbb{R}^{+}$.

Remark 5.11. As noticed by the author for small values of $N$ and confirmed in general by P. Johnson on Math Overflow, $a_{N-1}=(N-2)^{N-2}$. By (5.19),

$$
f(q)=1-e^{W(-q)}
$$

where $W \in \mathbb{Q}[[q]]$ is the Lambert $W$ function, i.e. the analytic function on a neighborhood of $0 \in \mathbb{C}$ defined by

$$
W(q) e^{W(q)}=q, \quad W(0)=0 .
$$

As can be seen from the Lagrange inversion formula,

$$
e^{W(q)}=1+q-\sum_{N=2}^{\infty} \frac{(N-1)^{(N-1)}}{N !}(-q)^{N}
$$

Along with (5.20), this implies the claim. 


\section{A Existence of Asymptotic Expansions}

In this appendix, we show that power series

$$
\mathcal{Y}_{0}(\hbar, \mathbf{x}, q) \equiv \sum_{d=0}^{\infty} q^{d} \frac{\prod_{k=1}^{k=l} \prod_{r=0}^{a_{k} d-1}\left(a_{k} \mathbf{x}+r \hbar\right)}{\prod_{r=1}^{r=d}\left(\prod_{k=1}^{k=n}\left(\mathbf{x}-\alpha_{k}+r \hbar\right)-\prod_{k=1}^{k=n}\left(\mathbf{x}-\alpha_{k}\right)\right)} \in \mathbb{Q}_{\alpha}(\mathbf{x}, \hbar)[[q]]
$$

admits an expansion of the form (4.15) and then prove Proposition 2.1. The arguments here are motivated by [26, Section 2].

Lemma A.1. The power series $\mathcal{Y}_{0}(\hbar, \mathbf{x}, q)$ admits an expansion of the form

$$
\mathcal{Y}_{0}(\hbar, \mathbf{x}, q)=e^{\xi(\mathbf{x}, q) / \hbar} \sum_{b=0}^{\infty} \Phi_{0 ; b}(\mathbf{x}, q) \hbar^{b}
$$

with $\xi, \Phi_{0 ; 1}, \Phi_{0 ; 2}, \ldots \in q \mathbb{Q}_{\alpha}(\mathbf{x})[[q]]$ and $\Phi_{0 ; 0} \in 1+q \mathbb{Q}_{\alpha}(\mathbf{x})[[q]]$.

Proof. Since $\mathcal{Y}_{0} \in 1+q \mathbb{Q}_{\alpha}(\hbar, \mathbf{x})[[q]]$, there is an expansion

$$
\ln \mathcal{Y}_{0}(\hbar, \mathbf{x}, q)=\sum_{d=1}^{\infty} \sum_{b=b_{\min }(d)}^{\infty} C_{d, b}(\mathbf{x}) \hbar^{b} q^{d}
$$

around $\hbar=0$, with $C_{d, b}(\mathbf{x}) \in \mathbb{Q}_{\alpha}(\mathbf{x})$; we can assume that $C_{d, b_{\min }(d)} \neq 0$ if $b_{\min }(d)<0$. The claim of Lemma A.1 is equivalent to the statement $b_{\min }(d) \geq-1$ for all $d \in \mathbb{Z}^{+}$; in such a case

$$
\xi(\mathbf{x}, q)=\sum_{d=1}^{\infty} C_{d,-1}(\mathbf{x}) q^{d}
$$

Suppose instead $b_{\min }(d)<-1$ for some $d \in \mathbb{Z}^{+}$. Let

$$
d^{*}=\min \left\{d \in \mathbb{Z}^{+}: b_{\min }(d)<-1\right\} \geq 1, \quad b^{*}=b_{\min }\left(d^{*}\right) \leq-2 .
$$

The power series $\mathcal{Y}_{0}$ satisfies the differential equation

$$
\left\{\prod_{k=1}^{k=n}\left(\mathbf{x}-\alpha_{k}+\hbar D\right)-q \prod_{k=1}^{l=1} \prod_{r=0}^{a_{k}-1}\left(a_{k} \mathbf{x}+a_{k} \hbar D+r \hbar\right)\right\} \mathcal{Y}_{0}(\hbar, \mathbf{x}, q)=\prod_{k=1}^{k=n}\left(\mathbf{x}-\alpha_{k}\right) \cdot \mathcal{Y}_{0}(\hbar, \mathbf{x}, q)
$$

where $D=q \frac{\mathrm{d}}{\mathrm{d} q}$. By (A.3), A.4 , and induction on the number of derivatives taken,

$$
\begin{gathered}
\frac{\left\{\prod_{k=1}^{k=n}\left(\mathbf{x}-\alpha_{k}+\hbar D\right)\right\} \mathcal{Y}_{0}(\hbar, \mathbf{x}, q)}{\prod_{k=1}^{k=n}\left(\mathbf{x}-\alpha_{k}\right) \cdot \mathcal{Y}_{0}(\hbar, \mathbf{x}, q)}=1+\sum_{k=1}^{k=n} \frac{d^{*} C_{d^{*}, b^{*}}}{\mathbf{x}-\alpha_{k}} \hbar^{b^{*}+1} q^{d^{*}}+A(\hbar, \mathbf{x}, q), \\
q \frac{\left\{\prod_{k=1}^{l=1} \prod_{r=0}^{a_{k}-1}\left(a_{k} \mathbf{x}+a_{k} \hbar D+r \hbar\right)\right\} \mathcal{Y}_{0}(\hbar, \mathbf{x}, q)}{\prod_{k=1}^{l=1} \prod_{r=0}^{a_{k}-1}\left(a_{k} \mathbf{x}+r \hbar\right) \cdot \mathcal{Y}_{0}(\hbar, \mathbf{x}, q)}=B(\hbar, \mathbf{x}, q),
\end{gathered}
$$


for some

$$
A, B \in q \mathbb{Q}_{\alpha}(\hbar, \mathbf{x})_{0}[[q]]+q^{d^{*}} \hbar^{b^{*}+2} \mathbb{Q}_{\alpha}(\hbar, \mathbf{x})_{0}[[q]]+q^{d^{*}+1} \mathbb{Q}_{\alpha}(\hbar, \mathbf{x})[[q]],
$$

where $\mathbb{Q}_{\alpha}(\hbar, \mathbf{x})_{0} \subset \mathbb{Q}_{\alpha}(\hbar, \mathbf{x})$ is the subring of rational functions in $\alpha$, $\hbar$, and $\mathbf{x}$ that are regular at $\hbar=0$. Combining (A.5) and (A.6), we conclude that $C_{d^{*}, b^{*}}=0$, contrary to the assumption.

Corollary A.2. The power series $F_{0} \in \mathbb{Q}(w)[[q]]$ defined by (2.15) admits an asymptotic expansion of the form (2.9).

Proof. The existence of an asymptotic expansion (2.9) is equivalent to the existence of an expansion of the form (4.15) for

$$
\left.F_{0}\left(\hbar^{-1}, q\right) \equiv \mathcal{Y}_{0}(\hbar, 1, q)\right|_{\alpha=0} .
$$

Thus, Corollary A.2 follows from Lemma A.1.

Remark A.3. It is possible to give a somewhat different proof of Corollary A.2, without using Lemma A.1, which is more in line with [26]. By [26, Lemma 1.3], an element $H \in \mathcal{P}$ admits an asymptotic expansion (2.9) if $\mathbf{M}^{k} H=H$ for some $k \in \mathbb{Z}^{+}$. By [23, Lemma 4.1], $\mathbf{M}^{n} F=F$ if $|\mathbf{a}|=n$. In the $\nu_{\mathbf{a}}>0$ case, the coefficients $\tilde{\mathrm{c}}_{p, s}^{(d)}$ in (2.18) with $d \geq 1$ and $\nu_{\mathbf{a}} d \leq p-s$ are determined by the requirement that the resulting function $F_{p}(w, q)$ is holomorphic at $w=0$ with value $1 \in Q[[q]]$; see (2.17). On the other hand, $F_{n}=F_{0}$ if these coefficients are given by

$$
\sum_{s=0}^{|\mathbf{a}|-l} \tilde{\mathrm{c}}_{n, l+s}^{(1)} w^{s}=-\langle\mathbf{a}\rangle \prod_{k=1}^{k=l} \prod_{r=1}^{a_{k}-1}\left(a_{k} w+r\right), \quad \tilde{\mathrm{c}}_{n, s}^{(d)}=0 \quad \forall d \geq 2 .
$$

Since $F_{0}$ is holomorphic at $w=0$ with value $1 \in Q[[q]]$, it follows that indeed $F_{n}=F_{0}$. The proof of [26. Lemma 1.3] can be adjusted to show that this in turn implies that $F_{0}$ admits an asymptotic expansion of the form (2.9).

In the remainder of this appendix, we prove Proposition 2.1 , Since $F=\mathbf{D}^{l} F_{0}$ and $F_{0}$ admits an asymptotic expansion of the form (2.9), so does $F$. The function $F(w, q)$ defined by (1.4) satisfies the ODE

$$
\left\{D_{w}^{n}-w^{n}-q w^{\nu_{\mathbf{a}}} \prod_{k=1}^{k=l} \prod_{r=1}^{r=a_{k}}\left(a_{k} D_{w}+r\right)\right\} F=0,
$$

where $D_{w}=w+q \frac{\mathrm{d}}{\mathrm{d} q}$. Thus, the power series $\xi, \Phi_{0}, \Phi_{1}, \ldots$ introduced in Proposition 2.1 satisfy

$$
\left\{\tilde{D}_{w}^{n}-w^{n}-q w^{\nu_{\mathbf{a}}} \prod_{k=1}^{k=l} \prod_{r=1}^{r=a_{k}}\left(a_{k} \tilde{D}_{w}+r\right)\right\} \sum_{b=0}^{\infty} \Phi_{b}(q) w^{-b}=0,
$$

where $\tilde{D}_{w}=\left(1+\xi^{\prime}(q)\right) w+q \frac{\mathrm{d}}{\mathrm{d} q}$. The resulting equation for the coefficient of $w^{n}$ gives

$$
\left\{\left(1+\xi^{\prime}(q)\right)^{n}-1-\mathbf{a}^{\mathbf{a}} q\left(1+\xi^{\prime}(q)\right)^{|\mathbf{a}|}\right\} \Phi_{0}(q)=0 .
$$

Since $\Phi_{0}(0)=1$, combining (A.8) with the condition $\xi^{\prime}(0)=0$ and comparing with (2.2), we obtain the first equation in (2.10).

By the above, $\tilde{D}_{w}=L(q) w+q \frac{\mathrm{d}}{\mathrm{d} q}$. Proceeding as in [26, Section 2.4], but using (5.7), we find that

$$
\tilde{D}_{w}^{s}=\sum_{k=0}^{k=s} \sum_{i=0}^{i=k}\left(\begin{array}{l}
s \\
i
\end{array}\right) \mathcal{H}_{s-i, k-i}\left(L^{n}\right)(L w)^{s-k} D^{i},
$$


where $\mathcal{H}_{m, j}$ are the rational functions defined by (2.5). Thus,

$$
L(q)^{n}\left\{\tilde{D}_{w}^{n}-w^{n}-q w^{\nu_{\mathbf{a}}} \prod_{k=1}^{k=l} \prod_{r=1}^{r=a_{k}}\left(a_{k} \tilde{D}_{w}+r\right)\right\}=\sum_{k=1}^{n}(L w)^{n-k} \mathfrak{L}_{k}
$$

where $\mathfrak{L}_{k}$ is the differential operator of order $k$ given by (2.7). It follows that the second equation in (2.10) is the coefficient of $(L w)^{n-1-b}$ in (A.7) multiplied by $L(q)^{n}$.

\section{B Some Combinatorics}

Lemma B.1. The following identities hold:

$$
\begin{array}{rlr}
\sum_{\mathbf{b}^{\prime} \in \mathcal{P}_{m}\left(b^{\prime}\right)} \prod_{i=1}^{i=m}\left(\begin{array}{c}
b_{i} \\
b_{i}^{\prime}
\end{array}\right)=\left(\begin{array}{c}
b_{1}+\ldots+b_{m} \\
b^{\prime}
\end{array}\right) & \forall m \in \mathbb{Z}^{+}, b_{1}, \ldots, b_{m}, b^{\prime} \in \overline{\mathbb{Z}}^{+}, \\
\sum_{b=0}^{\infty}(-1)^{b}\left(\begin{array}{c}
p \\
b
\end{array}\right) \prod_{t=B-s+1}^{t=B}(t+b)=(-1)^{p} s !\left(\begin{array}{c}
B \\
s-p
\end{array}\right) & \forall B, p, s \in \overline{\mathbb{Z}}^{+}, \\
\sum_{p=0}^{\infty}(-1)^{p}\left(\begin{array}{c}
m+p \\
p
\end{array}\right) \Psi^{p} & =\frac{1}{(1+\Psi)^{m+1}} & \forall m \in \overline{\mathbb{Z}}^{+} .
\end{array}
$$

The first two statements of this lemma are proved in [27, Appendix A]. The last statement is a special case of the Binomial Theorem; here is a direct argument:

$$
\begin{aligned}
\sum_{p=0}^{\infty}(-1)^{p}\left(\begin{array}{c}
m+p \\
p
\end{array}\right) \Psi^{p} & =\frac{1}{m !}\left\{\frac{\mathrm{d}}{\mathrm{d} \Psi}\right\}^{m} \sum_{p=0}^{\infty}(-1)^{p} \Psi^{m+p}=\frac{(-1)^{m}}{m !}\left\{\frac{\mathrm{d}}{\mathrm{d} \Psi}\right\}^{m} \sum_{p=0}^{\infty}(-1)^{p} \Psi^{p} \\
& =\frac{(-1)^{m}}{m !}\left\{\frac{\mathrm{d}}{\mathrm{d} \Psi}\right\}^{m} \frac{1}{1+\Psi}=\frac{1}{(1+\Psi)^{m+1}} .
\end{aligned}
$$

Lemma B.2. If $\zeta, \Psi_{0}, \Psi_{1}, \ldots \in Q \mathbb{Q}_{\alpha}(\hbar)[[Q]]$ and

$$
1+\mathcal{Z}^{*}(\hbar, Q)=e^{\zeta(Q) / \hbar}\left(1+\sum_{b=0}^{\infty} \Psi_{b}(Q) \hbar^{b}\right)
$$

then

$$
\begin{aligned}
& \sum_{m^{\prime}=0}^{\infty} \frac{\left(m^{\prime}+m\right) !}{m^{\prime} !} \sum_{\mathbf{b} \in \mathcal{P}_{m^{\prime}}\left(m-B+m^{\prime}\right)}\left(\prod_{k=1}^{k=m^{\prime}} \frac{(-1)^{b_{k}}}{b_{k} !} \Re \hbar_{\hbar=0}^{\Re}\left\{\hbar^{-b_{k}} \mathcal{Z}^{*}(\hbar, Q)\right\}\right) \\
& \quad=\sum_{\mathbf{c} \in\left(\overline{\mathbb{Z}}^{+}\right) \infty}\left((-1)^{|\mathbf{c}|+\|\mathbf{c}\|}(m+|\mathbf{c}|) ! \frac{\zeta(Q)^{B-m+\|\mathbf{c}\|}}{\left(1+\Psi_{0}(Q)\right)^{m+1}}\left(\begin{array}{c}
B \\
m-\|\mathbf{c}\|
\end{array}\right) \prod_{r=1}^{\infty} \frac{1}{c_{r} !}\left(\frac{\Psi_{r}(Q)}{(r+1) !\left(1+\Psi_{0}(Q)\right)}\right)^{c_{r}}\right)
\end{aligned}
$$

for all $m, B \in \overline{\mathbb{Z}}^{+}$.

Proof. If $\mathbf{c} \in\left(\overline{\mathbb{Z}}^{+}\right)^{\infty}$, let

$$
\Psi^{\mathbf{c}}=\prod_{r=1}^{\infty} \Psi_{r}^{c_{r}}, \quad \omega(\mathbf{c})=\prod_{r=1}^{\infty}((r+1) !)^{c_{r}}
$$


We show that each $\Psi_{0}^{c_{0}} \Psi^{\mathbf{c}}$, with $c_{0} \in \overline{\mathbb{Z}}^{+}$, enters with the same coefficient on the two sides of (B.2).

For $c_{0} \in \overline{\mathbb{Z}}^{+}$and $\mathbf{c} \in\left(\overline{\mathbb{Z}}^{+}\right)^{\infty}$, let

$$
S\left(c_{0}, \mathbf{c}\right)=\left\{(r, j) \in \overline{\mathbb{Z}}^{+} \times \mathbb{Z}^{+}:(r, j) \in\{r\} \times\left[c_{r}\right] \forall r \in \overline{\mathbb{Z}}^{+}\right\} .
$$

This is a finite set of cardinality $c_{0}+|\mathbf{c}|$. By (B.1), for all $b \in \overline{\mathbb{Z}}^{+}$

$$
\underset{\hbar=0}{\Re}\left\{\hbar^{-b} \mathcal{Z}^{*}(\hbar, Q)\right\}=\sum_{r=\max (b-1,0)}^{\infty} \frac{\zeta(Q)^{r+1-b}}{(r+1-b) !} \Psi_{r}(Q)+ \begin{cases}\zeta(Q), & \text { if } b=0 ; \\ 0, & \text { if } b>0 .\end{cases}
$$

Thus, for each $\mathbf{b} \in\left(\overline{\mathbb{Z}}^{+}\right)^{S\left(c_{0}, \mathbf{c}\right)}$ and every choice of disjoint subsets $S_{0}, S_{1}, \ldots$ of $\left[m^{\prime}\right]$, where

$$
m^{\prime}=B-m+|\mathbf{b}|,
$$

of cardinalities $c_{0}, c_{1}, \ldots$, the term $\Psi_{0}^{c_{0}} \Psi^{\mathbf{c}}$ appears in the $m^{\prime}$-th summand on the left-hand side of (B.2) with the coefficient

$$
\begin{aligned}
\frac{\left(m^{\prime}+m\right) !}{m^{\prime} !} \zeta^{m^{\prime}-c_{0}-|\mathbf{c}|} & \prod_{(r, j) \in S\left(c_{0}, \mathbf{c}\right)}\left(\frac{(-1)^{b_{r, j}}}{b_{r, j} !} \cdot \frac{\zeta^{r+1-b_{r, j}}}{\left(r+1-b_{r, j}\right) !}\right) \\
& =\frac{\zeta^{B-m+\|\mathbf{c}\|}}{\omega(\mathbf{c})}(-1)^{|\mathbf{b}|} \frac{\left(m^{\prime}+m\right) !}{m^{\prime} !} \prod_{(r, j) \in S\left(c_{0}, \mathbf{c}\right)}\left(\begin{array}{c}
r+1 \\
b_{r, j}
\end{array}\right) .15
\end{aligned}
$$

Since the number of above choices is

$$
\left(\begin{array}{c}
m^{\prime} \\
c_{0}, \mathbf{c}, m^{\prime}-c_{0}-|\mathbf{c}|
\end{array}\right) \equiv \frac{m^{\prime} !}{c_{0} ! \mathbf{c} !\left(m^{\prime}-c_{0}-|\mathbf{c}|\right) !},
$$

it follows that the coefficient of $\Psi_{0}^{c_{0}} \Psi^{\mathbf{c}}$ on the left-hand side of (B.2) is

$$
\frac{\zeta^{B-m+\|\mathbf{c}\|}}{\omega(\mathbf{c}) c_{0} ! \mathbf{c} !} \sum_{\mathbf{b} \in\left(\overline{\mathbb{Z}}^{+}\right)^{S\left(c_{0}, \mathbf{c}\right)}}\left((-1)^{|\mathbf{b}|} \prod_{t=B-m-c_{0}-|\mathbf{c}|+1}^{t=B}(t+|\mathbf{b}|) \prod_{(r, j) \in S\left(c_{0}, \mathbf{c}\right)}\left(\begin{array}{c}
r+1 \\
b_{r, j}
\end{array}\right)\right) .
$$

If $\left(c_{0}, \mathbf{c}\right)=(0, \mathbf{0})$ and thus $\left(\overline{\mathbb{Z}}^{+}\right)^{S\left(c_{0}, \mathbf{c}\right)} \equiv\{\mathbf{0}\}$, this expression reduces to $m !\left(\begin{array}{l}B \\ m\end{array}\right) \zeta^{B-m}$. Otherwise, (B.4) becomes

$$
\begin{aligned}
& \frac{\zeta^{B-m+\|\mathbf{c}\|}}{c_{0} ! \mathbf{c} ! \omega(\mathbf{c})} \sum_{b=0}^{\infty}\left((-1)^{b}\left(\begin{array}{c}
c_{0}+|\mathbf{c}|+\|\mathbf{c}\| \\
b
\end{array}\right) \prod_{t=B-m-c_{0}-|\mathbf{c}|+1}^{t=B}(t+b)\right. \\
& =\frac{\zeta^{B-m+\|\mathbf{c}\|}}{c_{0} ! \mathbf{c} ! \omega(\mathbf{c})}(-1)^{c_{0}+|\mathbf{c}|+\|\mathbf{c}\|}\left(m+c_{0}+|\mathbf{c}|\right) !\left(\begin{array}{c}
B \\
m-\|\mathbf{c}\|
\end{array}\right),
\end{aligned}
$$

by the first two statements of Lemma B.1. Lemma B.2 now follows from the last statement of Lemma B.1.

\footnotetext{
${ }^{15}$ The factors in the $m^{\prime}$-fold product in $(\underline{\mathrm{B} .2})$ that contribute $\Psi_{r}$ are indexed by the elements of $S_{r}$; the $j$-th such factor arises from $\underset{\hbar=0}{\mathfrak{R}}\left\{\hbar^{-b_{r, j}} \mathcal{Z}^{*}(\hbar, Q)\right\}$ with $r \geq b_{r, j}-1$. This leaves $m^{\prime}-c_{0}-|\mathbf{c}|$ factors that contribute $\zeta(Q)$ from $\underset{\hbar=0}{\mathfrak{R}}\left\{\mathcal{Z}^{*}(\hbar, Q)\right\}$. The first expression in (B.3) is defined to be 0 if $b_{r, j}>r+1$ for some $(r, j) \in S\left(c_{0}, \mathbf{c}\right)$.
} 
For any $d \in \overline{\mathbb{Z}}^{+}$and $t \in \mathbb{Z}$, let

$$
\left(\begin{array}{l}
t \\
d
\end{array}\right)=\frac{\prod_{r=0}^{d-1}(t-r)}{d !}
$$

For $r \in \overline{\mathbb{Z}}^{+}$and $\mathbf{p} \in\left(\overline{\mathbb{Z}}^{+}\right)^{n}$, define $w_{r} \in \mathbb{Q}[\alpha]$ and $C_{r ; \mathbf{p}} \in \mathbb{Q}$ by

$$
w_{r} \equiv \sum_{i=1}^{i=n} \alpha_{i}^{r} \equiv \sum_{\mathbf{p} \in\left(\overline{\mathbb{Z}}^{+}\right)^{n}} C_{r ; \mathbf{p}} \hat{\sigma}_{1}^{p_{1}} \hat{\sigma}_{2}^{p_{2}} \ldots \hat{\sigma}_{n}^{p_{n}}
$$

If $r_{1}, r_{2} \in[n]$ with $r_{1} \neq r_{2}$ and $b_{1}, b_{2} \in \overline{\mathbb{Z}}^{+}$, let

$$
\mathbf{p}=\left(p_{1}, \ldots, p_{n}\right), \quad p_{r}=\left\{\begin{array}{ll}
b_{i}, & \text { if } r=r_{i} ; \\
0, & \text { otherwise; }
\end{array} \quad C_{r_{1}, r_{2}}^{\left(b_{1}, b_{2}\right)}=C_{b_{1} r_{1}+b_{2} r_{2} ; \mathbf{p}}\right.
$$

Thus, $C_{r_{1}, r_{2}}^{\left(b_{1}, b_{2}\right)}$ is the coefficient of $\hat{\sigma}_{r_{1}}^{b_{1}} \hat{\sigma}_{r_{2}}^{b_{2}}$ in the expansion of $w_{b_{1} r_{1}+b_{2} r_{2}}$ in terms of products of the modified (by sign) elementary symmetric polynomials $\hat{\sigma}_{r}$. If $b_{1}<0$ or $b_{2}<0$, set $C_{r_{1}, r_{2}}^{\left(b_{1}, b_{2}\right)}=0$.

Lemma B.3. If $r_{1}, r_{2} \in[n]$ with $r_{1} \neq r_{2}$ and $b_{1}, b_{2} \in \overline{\mathbb{Z}}^{+}$with $b_{1}+b_{2} \neq 0$,

$$
C_{r_{1}, r_{2}}^{\left(b_{1}, b_{2}\right)}=\left(\begin{array}{c}
b_{1}+b_{2}-1 \\
b_{2}
\end{array}\right) r_{1}+\left(\begin{array}{c}
b_{1}+b_{2}-1 \\
b_{1}
\end{array}\right) r_{2}
$$

Proof. If $b_{1} \in \mathbb{Z}^{+}$and $\alpha_{1}, \ldots, \alpha_{n}$ are the $n$ roots of the polynomial $\alpha^{n}-\alpha^{n-r_{1}}=\alpha^{n-r_{1}}\left(\alpha^{r_{1}}-1\right)$,

$$
C_{r_{1}, r_{2}}^{\left(b_{1}, 0\right)}=\sum_{i=1}^{i=n} \alpha_{i}^{b_{1} r_{1}}=r_{1} \cdot 1^{b_{1}}+\left(n-r_{1}\right) \cdot 0^{b_{1} r_{1}}=r_{1}
$$

thus, the claim holds when either $b_{1}=0$ or $b_{2}=0$. If $b_{1}, b_{2} \in \mathbb{Z}^{+}$,

$$
w_{b_{1} r_{1}+b_{2} r_{2}}=\sum_{r=1}^{b_{1} r_{1}+b_{2} r_{2}-1} \hat{\sigma}_{r} w_{b_{1} r_{1}+b_{2} r_{2}-r}+\left(b_{1} r_{1}+b_{2} r_{2}\right) \hat{\sigma}_{b_{1} r_{1}+b_{2} r_{2}}
$$

by Newton's identity [2, p577]. This gives

$$
C_{r_{1}, r_{2}}^{\left(b_{1}, b_{2}\right)}=C_{r_{1}, r_{2}}^{\left(b_{1}-1, b_{2}\right)}+C_{r_{1}, r_{2}}^{\left(b_{1}, b_{2}-1\right)} \quad \forall b_{1}, b_{2} \in \mathbb{Z}^{+} .
$$

Along with the $b_{1}=0$ or $b_{2}=0$ case, this implies the claim by induction.

Lemma B.4. The power series $L \in 1+q \mathbb{Q}[[q]]$ defined by (2.2) satisfies

$$
\llbracket \frac{n L(q)^{\nu_{\mathbf{a}} d+n t}}{|\mathbf{a}|+\nu_{\mathbf{a}} L(q)^{n}} \rrbracket_{q ; d}=\left(\mathbf{a}^{\mathbf{a}}\right)^{d}\left(\begin{array}{c}
d+t-1 \\
d
\end{array}\right)
$$

for all $d \in \overline{\mathbb{Z}}^{+}$and $t \in \mathbb{Z}$. 
Proof. In the two extremal cases, by (2.3)

$$
\frac{n L(q)^{\nu_{\mathbf{a}} d+n t}}{|\mathbf{a}|+\nu_{\mathbf{a}} L(q)^{n}}= \begin{cases}(1+q)^{d+t-1}, & \text { if }|\mathbf{a}|=0 \\ \left(1-\mathbf{a}^{\mathbf{a}} q\right)^{-t}, & \text { if }|\mathbf{a}|=n .\end{cases}
$$

Thus, the claim in these two cases follows from the binomial theorem; so, we can assume that $0<|\mathbf{a}|<n$. Replacing $\mathbf{a}^{\mathbf{a}} q$ by $q$ in (2.2), we observe that it is sufficient to prove (B.4) with $L$ defined by (2.2) with $\mathbf{a}^{\mathbf{a}}$ replaced by 1 and $|\mathbf{a}|$ by some $a \in \mathbb{Z}^{+}$with $a<n$; thus, $\nu_{\mathbf{a}}=\nu \equiv n-a$.

With these reductions, for each $n$-th root of unity $\zeta \in \mathbb{C}$ let

$$
L_{\zeta}(q)=\zeta L\left(\zeta^{a} q\right) \in \mathbb{Q}[[q]]
$$

Then,

$$
\begin{aligned}
L_{\zeta}(q)^{n}-q L_{\zeta}(q)^{a}=1 & \Longrightarrow \frac{1}{a+\nu L_{\zeta}(q)^{n}}=\frac{L_{\zeta}^{\prime}(q)}{q L_{\zeta}(q)^{a+1}} \\
\zeta^{\nu d+n t} \cdot\left(\zeta^{a} q\right)^{d}=q^{d} & \Longrightarrow \llbracket \frac{n L(q)^{\nu d+n t}}{a+\nu L(q)^{n}} \rrbracket_{q ; d}=\sum_{\zeta^{n}=1} \llbracket \frac{L_{\zeta}(q)^{\nu d+n t}}{a+\nu L_{\zeta}(q)^{n}} \rrbracket_{q ; d},
\end{aligned}
$$

where' denotes $q \frac{\mathrm{d}}{\mathrm{d} q}$ as before. Combining these two conclusions, we find that

$$
\llbracket \frac{n L(q)^{\nu d+n t}}{a+\nu L(q)^{n}} \rrbracket_{q ; d}=\sum_{\zeta^{n}=1} \llbracket L_{\zeta}(q)^{\nu(d+1)+n(t-1)} \frac{L_{\zeta}^{\prime}(q)}{L_{\zeta}(q)} \rrbracket_{q ; d+1} .
$$

If $\nu(d+1)+n(t-1)=0$, this gives

$$
\begin{aligned}
\llbracket \frac{n L(q)^{\nu d+n t}}{a+\nu L(q)^{n}} \rrbracket_{q ; d}=(d+1) \sum_{\zeta^{n}=1} \llbracket \ln L_{\zeta}(q) \rrbracket_{q ; d+1} & =(d+1) \llbracket \ln \left(\prod_{\zeta^{n}=1} L_{\zeta}(q)\right) \rrbracket_{q ; d+1} \\
& =(d+1) \llbracket \ln (-1)^{n-1} \rrbracket_{q ; d+1}=0,
\end{aligned}
$$

since $\left\{L_{\zeta}\right\}_{\zeta^{n}=1}$ is the set of the roots of $\ell^{n}-q \ell^{a}-1=0$. Since $\nu<n$, our assumption on $(d, t)$ implies that $0 \leq d+t-1<d$, and so the right-hand side of (B.7) also vanishes. If $\nu(d+1)+n(t-1)>0$, (B.8) and Lemma B.3 give

$$
\begin{aligned}
\llbracket \frac{n L(q)^{\nu d+n t}}{a+\nu L(q)^{n}} \rrbracket_{q ; d} & =\frac{d+1}{\nu(d+1)+n(t-1)} \sum_{\zeta^{n}=1} \llbracket L_{\zeta}(q)^{\nu(d+1)+n(t-1)} \rrbracket_{q ; d+1} \\
& =\frac{d+1}{\nu(d+1)+n(t-1)} C_{\nu, n}^{(d+1, t-1)}=\left(\begin{array}{c}
d+t-1 \\
d
\end{array}\right),
\end{aligned}
$$

as claimed (the last equality holds even if $t \leq 0)$. If $\nu(d+1)+n(t-1)<0,(\underline{\mathrm{B} .8})$ and Lemma B.3 give

$$
\begin{aligned}
\llbracket \frac{n L(q)^{\nu d+n t}}{a+\nu L(q)^{n}} \rrbracket_{q ; d} & =\frac{d+1}{\nu(d+1)+n(t-1)} \sum_{\zeta^{n}=1} \llbracket\left(\frac{1}{L_{\zeta}(q)}\right)^{a(d+1)-n(d+t)} \rrbracket_{q ; d+1} \\
& =\frac{d+1}{\nu(d+1)+n(t-1)} C_{a, n}^{(d+1,-(d+t))}(-1)^{d+1}=(-1)^{d}\left(\begin{array}{c}
-t \\
d
\end{array}\right)
\end{aligned}
$$


since $\left\{1 / L_{\zeta}\right\}_{\zeta^{n}=1}$ is the set of the roots of $\ell^{n}+q \ell^{\nu}-1=0$; the last equality holds even if $d+t>0$. Since

$$
(-1)^{d}\left(\begin{array}{c}
-t \\
d
\end{array}\right)=\left(\begin{array}{c}
d+t-1 \\
d
\end{array}\right)
$$

(B.7) holds in this last case as well.

Corollary B.5. The power series $L \in 1+q \mathbb{Q}[[q]]$ defined by (2.2) satisfies

$$
\llbracket \frac{n L(q)^{\nu_{\mathbf{a}} d+n t}}{\left(|\mathbf{a}|+\nu_{\mathbf{a}} L(q)^{n}\right)^{k}} \cdot \frac{L^{\prime}(q)}{L(q)} \rrbracket_{q ; d}=\frac{\left(\mathbf{a}^{\mathbf{a}}\right)^{d}}{n^{k}} \sum_{r=0}^{d-1}\left(\begin{array}{c}
k-1+r \\
r
\end{array}\right)\left(\begin{array}{c}
d-1+t \\
d-1-r
\end{array}\right)\left(-\frac{\nu_{\mathbf{a}}}{n}\right)^{r}
$$

for all $d \in \overline{\mathbb{Z}}^{+}$and $k, t \in \mathbb{Z}$.

Proof. For $d=0$, both sides of (B.9) vanish. By (5.7), the $k=0$ case of (B.9) reduces to Lemma B.4. For $k \neq 0$, by (5.7) and the Binomial Theorem

$$
\begin{aligned}
\llbracket \frac{n L(q)^{\nu_{\mathbf{a}} d+n t}}{\left(|\mathbf{a}|+\nu_{\mathbf{a}} L(q)^{n}\right)^{k}} \cdot \frac{L^{\prime}(q)}{L(q)} \rrbracket_{q ; d} & =\mathbf{a}^{\mathbf{a}} \llbracket \frac{n L(q)^{\nu_{\mathbf{a}}(d-1)+n(t+1)}}{|\mathbf{a}|+\nu_{\mathbf{a}} L(q)^{n}} \cdot \frac{1}{\left(n+\nu_{\mathbf{a}} \mathbf{a}^{\mathbf{a}} q L(q)^{|\mathbf{a}|)^{k}}\right.} \rrbracket_{q ; d-1} \\
& =\frac{\mathbf{a}^{\mathbf{a}}}{n^{k}} \sum_{r=0}^{d-1}\left(\begin{array}{c}
-k \\
r
\end{array}\right) \llbracket \frac{n L(q)^{\nu_{\mathbf{a}}(d-1-r)+n(t+1+r)}}{|\mathbf{a}|+\nu_{\mathbf{a}} L(q)^{n}} \rrbracket_{q ; d-1-r}\left(\frac{\nu_{\mathbf{a}}}{n} \mathbf{a}^{\mathbf{a}}\right)^{r} .
\end{aligned}
$$

The claim now follows from Lemma B.4.

For $p, d \in \mathbb{Z}$, let $\llbracket p \rrbracket_{d}, \llbracket \hat{p} \rrbracket_{d}, \tau_{d}(p), t_{d}(p) \in \mathbb{Z}$ be as in (2.41). In particular,

$$
\begin{aligned}
& \left(\tau_{d-1}(p)-\tau_{d}(p), t_{d}(p)\right) \in\{(0,0),(1,0),(0,1)\}, \\
& 1-t_{1}(p)-\tau_{0}(p)+\tau_{1}(p)= \begin{cases}1, & \text { if } t_{1}(p)=0 \text { and } \tau_{0}(p)=\tau_{1}(p) ; \\
0, & \text { otherwise. }\end{cases}
\end{aligned}
$$

Let $A=\mathbf{a}^{\mathbf{a}}$ for the remainder this section.

Lemma B.6. For all $d \in \overline{\mathbb{Z}}^{+}, p \in \mathbb{Z}$, and $f: \mathbb{Z}^{2} \longrightarrow \mathbb{R}$,

$$
\begin{aligned}
& \sum_{\substack{d_{1}+d_{2}=d \\
d_{1}, d_{2} \geq 0}} \tilde{\mathrm{c}}_{\llbracket p \rrbracket_{d_{2}}, \llbracket p \rrbracket_{d_{2}}-\nu_{\mathbf{a}} d_{1} \tilde{\mathrm{c}}_{\llbracket \hat{p} \rrbracket_{d_{2}}, \llbracket \hat{p} \rrbracket_{d_{2}}-\nu_{\mathbf{a}} d_{2}}^{\left(d_{1}\right)}}^{\tilde{c}_{d_{2}}} f\left(\tau_{d_{2}}(p), t_{d_{2}}(p)\right) \\
& = \begin{cases}f\left(\tau_{0}(p), t_{0}(p)\right), & \text { if } d=0 ; \\
-A\left(1-\tau_{0}(p)+\tau_{1}(p)-t_{1}(p)\right) f\left(\tau_{1}(p), t_{1}(d)\right), & \text { if } d=1 ; \\
0, & \text { if } d \geq 2 .\end{cases}
\end{aligned}
$$

Proof. The $d=0$ case of $(\underline{\mathrm{B} .12})$ is immediate from $\tilde{\mathrm{c}}_{p, s}^{(0)}=\delta_{p, s}$. If $\tau_{0}(p)=\tau_{d}(p)$ and $t_{d}(p)=0$, (B.12) reduces to $[24,(2.9)]$. In general, let $d_{1}^{*}, \ldots, d_{k}^{*} \in \mathbb{Z}^{+}$be such that

$$
\tau_{0}(p)=\tau_{d_{1}^{*}-1}(p)>\tau_{d_{1}^{*}}(p)=\tau_{d_{2}^{*}-1}(p)>\tau_{d_{2}^{*}}(p)=\tau_{d_{3}^{*}-1}(p) \ldots>\tau_{d_{k}^{*}}(p)=\tau_{d}(p) ;
$$

if $\tau_{0}(p)=\tau_{d}(p), k \equiv 0$. Let $d_{0}^{*}=0$ and $d_{k+1}^{*}=d+1$. If $1 \leq i \leq k$, then $\llbracket p \rrbracket_{d_{i}^{*}-1}<\nu_{\mathbf{a}}, \llbracket \hat{p} \rrbracket_{d_{i}^{*}}<l+\nu_{\mathbf{a}}$, and so

$$
\begin{array}{lllll}
d_{i-1}^{*} \leq d_{2}<d_{i}^{*} & \Longrightarrow & \llbracket p \rrbracket_{d_{2}}-\nu_{\mathbf{a}}\left(d-d_{2}\right)<0 & \Longrightarrow & \tilde{\mathrm{c}}_{\llbracket p \rrbracket_{d_{2}}, \llbracket p \rrbracket_{d_{2}}-\nu_{\mathbf{a}}\left(d-d_{2}\right)}^{\left(d-d_{2}\right)}=0 ; \\
d_{i}^{*} \leq d_{2}<d_{i+1}^{*} & \Longrightarrow & \llbracket \hat{p} \rrbracket_{d_{2}}-\nu_{\mathbf{a}} d_{2}<l & \Longrightarrow & \tilde{\mathrm{c}}_{\llbracket \hat{p} \rrbracket_{d_{2}}, \llbracket \tilde{p} \rrbracket_{d_{2}}-\nu_{\mathbf{a}} d_{2}}^{\left(d_{2}\right.}=0 .
\end{array}
$$


Thus, all summands on the left-hand side of (B.12) vanish if $k \neq 0$. Finally, if $d>0$ and $k=0$, but $t_{d}(p)=1$, then $\llbracket p \rrbracket_{d}, \llbracket \hat{p} \rrbracket_{d}<l$, and so

$$
\tilde{\mathrm{c}}_{\llbracket \hat{p} \rrbracket_{d}, \llbracket \hat{p} \rrbracket_{d}-\nu_{\mathbf{a}} d}^{(d)}=0 ; \quad \llbracket p \rrbracket_{d_{2}}-\nu_{\mathbf{a}}\left(d-d_{2}\right)<l \quad \Longrightarrow \quad \tilde{\mathrm{c}}_{\llbracket p \rrbracket_{d_{2}}, \llbracket p \rrbracket_{d_{2}}-\nu_{\mathbf{a}}\left(d-d_{2}\right)}^{\left(d-d_{2}\right)}=0 \quad \forall d_{2}=0,1, \ldots, d-1 .
$$

Thus, all summands on the left-hand side of (B.12) vanish in this case as well. In light of (B.11), this confirms (B.12).

Lemma B.7. For all $d \in \overline{\mathbb{Z}}^{+}$and $p \in \mathbb{Z}$,

$$
\begin{aligned}
& \sum_{\mathbf{d} \in \mathcal{P}_{4}(d)}\left\{\tilde{\mathrm{c}}_{\llbracket p \rrbracket_{d_{2}+d_{3}}, \llbracket p \rrbracket_{d_{2}+d_{3}}-\nu_{\mathbf{a}} d_{1}}^{\left(d_{1}\right)} \tilde{\mathrm{c}}_{\llbracket \hat{p} \rrbracket_{d_{2}+d_{3}}, \llbracket \hat{\Perp} \rrbracket_{d_{2}+d_{3}}-\nu_{\mathbf{a}} d_{2}}^{\left(d_{2}\right)}\right. \\
& \left.A^{d_{3}}\left(\begin{array}{c}
d_{3}+\tau_{d_{2}+d_{3}}(p)-t_{d_{2}+d_{3}}(p) \\
d_{3}
\end{array}\right) \llbracket \frac{n L(q)^{\nu_{\mathbf{a}} d_{4}-n \tau_{d_{2}+d_{3}}(p)}}{|\mathbf{a}|+\nu_{\mathbf{a}} L(q)^{n}} \|_{q ; d_{4}}\right\}=\delta_{d, 0} .
\end{aligned}
$$

Proof. The $d=0$ case is clear; so we assume $d>0$. Using Lemma B.6 to sum over $d_{1}+d_{2}=d^{\prime}$ with $d^{\prime}$ fixed, we find that the left-hand side of (B.7) equals

$$
\begin{aligned}
& \llbracket \frac{n L(q)^{\nu_{\mathbf{a}} d-n \tau_{0}(p)}}{|\mathbf{a}|+\nu_{\mathbf{a}} L(q)^{n}} \|_{q ; d} \\
& +\sum_{\substack{d_{3}+d_{4}=d \\
1<d_{3}<d}} A^{d_{3}}\left(\begin{array}{c}
d_{3}-1+\tau_{d_{3}}(p)-t_{d_{3}}(p) \\
d_{3}-1
\end{array}\right) \frac{d_{3} \tau_{d_{3}-1}(p)+\left(d_{3}-1\right)\left(t_{d_{3}}(p)-\tau_{d_{3}}(p)\right)}{d_{3}} \llbracket \frac{n L(q)^{\nu_{\mathbf{a}} d_{4}-n \tau_{d_{3}}(p)}}{|\mathbf{a}|+\nu_{\mathbf{a}} L(q)^{n}} \|_{q ; d_{4}} .
\end{aligned}
$$

By (B.10),

$$
\left(\begin{array}{c}
d_{3}-1+\tau_{d_{3}}(p)-t_{d_{3}}(p) \\
d_{3}-1
\end{array}\right) \frac{d_{3} \tau_{d_{3}-1}(p)+\left(d_{3}-1\right)\left(t_{d_{3}}(p)-\tau_{d_{3}}(p)\right)}{d_{3}}=\left(\begin{array}{c}
d_{3}-1+\tau_{d_{3}-1}(p) \\
d_{3}
\end{array}\right) .
$$

It follows that the left-hand side of (B.13) equals

$$
A^{d}\left(\begin{array}{c}
d-1-\tau_{0}(p) \\
d
\end{array}\right)+A^{d} \sum_{\substack{d_{3}+d_{4}=d \\
1 \leq d_{3} \leq d}}\left(\begin{array}{c}
d_{3}-1+\tau_{d_{3}-1}(p) \\
d_{3}
\end{array}\right)\left(\begin{array}{c}
d_{4}-1-\tau_{d_{3}}(p) \\
d_{4}
\end{array}\right)
$$

see also Lemma B.4. By induction on $s=0,1, \ldots, d-1$,

$$
\sum_{\substack{d_{3}+d_{4}=d \\
d-s \leq d_{3} \leq d}}\left(\begin{array}{c}
d_{3}-1+\tau_{d_{3}-1}(p) \\
d_{3}
\end{array}\right)\left(\begin{array}{c}
d_{4}-1-\tau_{d_{3}}(p) \\
d_{4}
\end{array}\right)=(-1)^{s}\left(\begin{array}{c}
d-1 \\
s
\end{array}\right)\left(\begin{array}{c}
d-1-s+\tau_{d-1-s}(p) \\
d
\end{array}\right) .
$$

Setting $s=d-1$ in the last identity, we conclude that the sum in (B.14) vanishes.

Corollary B.8. For all $d \in \overline{\mathbb{Z}}^{+}, p, t \in \mathbb{Z}$, and $f \in \mathbb{R}[[q]]$,

$$
\begin{aligned}
\sum_{\mathbf{d} \in \mathcal{P}_{4}(d)}\left\{\tilde{\mathrm{c}}_{\llbracket p \rrbracket_{d_{2}+d_{3}}, \llbracket p \rrbracket_{d_{2}+d_{3}}-\nu_{\mathbf{a}} d_{1}}^{\left(d_{1}\right)} \tilde{\mathrm{c}}_{\llbracket \hat{p} \rrbracket_{d_{2}+d_{3}}, \llbracket \hat{p} \rrbracket_{d_{2}+d_{3}}-\nu_{\mathbf{a}} d_{2}}^{\left(d_{2}\right)}\left(\mathbf{a}^{\mathbf{a}}\right)^{d_{3}}\left(\begin{array}{c}
d_{3}+\tau_{d_{2}+d_{3}}(p)-t_{d_{2}+d_{3}}(p)-t \\
d_{3}
\end{array}\right)\right. \\
\left.\times \llbracket \frac{n L(q)^{\nu_{\mathbf{a}} d_{4}+n\left(t-\tau_{d_{2}+d_{3}}(p)\right)}}{|\mathbf{a}|+\nu_{\mathbf{a}} L(q)^{n}} f(q) \rrbracket_{q ; d_{4}}\right\}=\llbracket \frac{n L(q)^{\nu_{\mathbf{a}} d} f(q)}{|\mathbf{a}|+\nu_{\mathbf{a}} L(q)^{n}} \rrbracket_{q ; d} .
\end{aligned}
$$


Proof. Replacing $p$ with $p-n t$, we can assume that $t=0$. The $d=0$ case is clear; so we assume that $d \geq 1$ and that the above identity holds with $d$ replaced by any nonnegative integer $d^{\prime}<d$. The left-hand side of this identity is given by

$$
\begin{aligned}
& \mathrm{LHS}_{d}=\sum_{\begin{array}{c}
\prime \\
d^{\prime}+d^{\prime \prime}=d \\
d^{\prime}, d^{\prime \prime} \geq 0
\end{array}} C_{d^{\prime}, d^{\prime \prime}} \llbracket f(q) \rrbracket_{q ; d^{\prime \prime}}, \quad \text { where }
\end{aligned}
$$

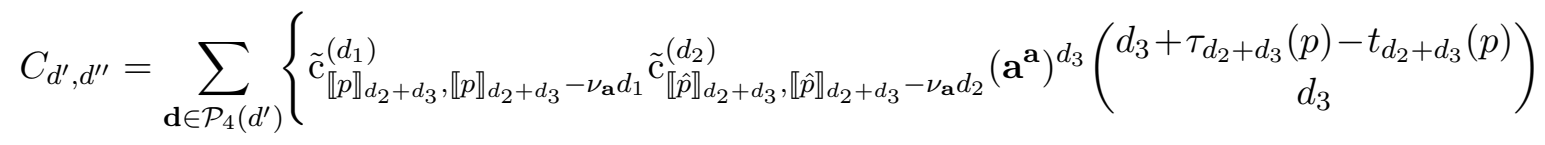

$$
\begin{aligned}
& \left.\times \llbracket \frac{n L(q)^{\nu_{\mathbf{a}}\left(d_{4}+d^{\prime \prime}\right)-n \tau_{d_{2}+d_{3}}(p)}}{|\mathbf{a}|+\nu_{\mathbf{a}} L(q)^{n}} \rrbracket_{q ; d_{4}}\right\} .
\end{aligned}
$$

So, it is sufficient to show that

$$
C_{d^{\prime}, d^{\prime \prime}}=\llbracket \frac{n L(q)^{\nu_{\mathbf{a}} d}}{|\mathbf{a}|+\nu_{\mathbf{a}} L(q)^{n}} \rrbracket_{q ; d^{\prime}}
$$

for $d^{\prime}=0,1, \ldots, d$. For $d^{\prime}<d$, this is the case by the inductive assumption applied with $f=L^{\nu_{\mathbf{a}} d^{\prime \prime}}$. For $d^{\prime}=d^{\prime \prime}$, this is the case by Lemmas B.7 and B.4.

Department of Mathematics, SUNY Stony Brook, Stony Brook, NY 11794-3651

azinger@math.sunysb.edu

\section{References}

[1] T. Apostol, Calculus, Vol. II, Wiley 1969.

[2] M. Artin, Algebra, Prentice Hall, 1991.

[3] M. Atiyah and R. Bott, The moment map and equivariant cohomology, Topology 23 (1984), $1-28$.

[4] S. Barannikov, Generalized periods and mirror symmetry in dimensions $n>3$, math/9903124.

[5] A. Bertram, Another way to enumerate rational curves with torus actions, Invent. Math. 142 (2000), no. 3, 487-512.

[6] P. Candelas, X. de la Ossa, P. Green, and L. Parkes, A pair of Calabi-Yau manifolds as an exactly soluble superconformal theory, Nuclear Phys. B359 (1991), 21-74.

[7] L. Cherveny, Genus-zero mirror principle with two marked points, math/1001.0242.

[8] G. Ellingsrud and S. Stromme, Bott's formula and enumerative geometry, JAMS 9 (1996), 175-193.

[9] A. Gathmann, Absolute and relative Gromov-Witten invariants of very ample hypersurfaces, Duke Math. J. 115 (2002), no. 2, 171-203.

[10] A. Givental, Equivariant Gromov-Witten invariants, IMRN no. 13 (1996), 613-663. 
[11] A. Givental, The mirror formula for quintic threefolds, Amer. Math. Soc. Transl. Ser. 2, 196 (1999), 49-62.

[12] A. Givental, Semisimple Frobenius structures at higher genus, IMRN 2001, no. 23, 1265-1286.

[13] B. Greene, D. Morrison, and M. Plesser, Mirror manifolds in higher dimension, Mirror Symmetry, II, 745-791, AMS/IP Stud. Adv. Math. 1, 1997.

[14] P. Griffiths and J. Harris, Principles of Algebraic Geometry, John Willey \& Sons, 1994.

[15] K. Hori, S. Katz, A. Klemm, R. Pandharipande, R. Thomas, C. Vafa, R. Vakil, and E. Zaslow, Mirror Symmetry, Clay Math. Inst., Amer. Math. Soc., 2003.

[16] S. Katz, Rational curves on Calabi-Yau manifolds: verifying predictions of mirror symmetry, Algebraic Geometry (E. Ballico, ed.), Marcel-Dekker, New York, 1994.

[17] A. Klemm and R. Pandharipande, Enumerative geometry of Calabi-Yau 4-folds, math.AG/0702189.

[18] Y. P. Lee, Quantum Lefschetz hyperplane theorem, Invent. Math. 145 (2001), no. 1, 121-149.

[19] B. Lian, K. Liu, and S.T. Yau, Mirror Principle I, Asian J. of Math. 1, no. 4 (1997), 729-763.

[20] D. Maulik and R. Pandharipande, A topological view of Gromov-Witten theory, Topology 45 (2006), no. 5, 887-918.

[21] D. Maulik and R. Pandharipande, in progress.

[22] D. McDuff and D. Salamon, J-holomorphic Curves and Symplectic Topology, AMS 2004.

[23] A. Popa, The genus one Gromov-Witten invariants of Calabi-Yau complete intersections, math/1007.3534, to appear in TAMS.

[24] A. Popa and A. Zinger, On mirror formulas in open and closed Gromov-Witten theory, math/1010.1946.

[25] Y. Ruan and G. Tian, A mathematical theory of quantum cohomology, JDG 42 (1995), no. 2, 259-367.

[26] D. Zagier and A. Zinger, Some properties of hypergeometric series associated with mirror symmetry, Modular Forms and String Duality, Fields Inst. Commun. 54 (2008), 163-177.

[27] A. Zinger, The reduced genus-one Gromov-Witten invariants of Calabi-Yau hypersurfaces, J. Amer. Math. Soc. 22 (2009), no. 3, 691-737.

[28] A. Zinger, Genus-zero two-point hyperplane integrals in the Gromov-Witten theory, Comm. Ann. Geom. 17 (2010), no. 5, 1-45. 